Article

\title{
Insights into the Evolution of a Snake Venom Multi-Gene Family from the Genomic Organization of Echis ocellatus SVMP Genes
}

\author{
Libia Sanz * and Juan J. Calvete * \\ Laboratorio de Venómica Estructural y Funcional, Instituto de Biomedicina de Valencia, \\ Consejo Superior de Investigaciones Científicas, Jaume Roig 11, 46010 València, Spain \\ * Correspondence: libia.sanz@ibv.csic.es (L.S.); jcalvete@ibv.csic.es (J.J.C.); Tel.: +34-96-339-1760 (L.S.); \\ +34-96-339-1778 (J.J.C.)
}

Academic Editors: Jay Fox and José María Gutiérrez

Received: 12 June 2016; Accepted: 6 July 2016; Published: 12 July 2016

\begin{abstract}
The molecular events underlying the evolution of the Snake Venom Metalloproteinase (SVMP) family from an A Disintegrin And Metalloproteinase (ADAM) ancestor remain poorly understood. Comparative genomics may provide decisive information to reconstruct the evolutionary history of this multi-locus toxin family. Here, we report the genomic organization of Echis ocellatus genes encoding SVMPs from the PII and PI classes. Comparisons between them and between these genes and the genomic structures of Anolis carolinensis ADAM28 and E. ocellatus PIII-SVMP EOC00089 suggest that insertions and deletions of intronic regions played key roles along the evolutionary pathway that shaped the current diversity within the multi-locus SVMP gene family. In particular, our data suggest that emergence of EOC00028-like PI-SVMP from an ancestral PII(e/d)-type SVMP involved splicing site mutations that abolished both the $3^{\prime}$ splice AG acceptor site of intron $12^{*}$ and the $5^{\prime}$ splice GT donor site of intron $13^{*}$, and resulted in the intronization of exon $13^{*}$ and the consequent destruction of the structural integrity of the PII-SVMP characteristic disintegrin domain.
\end{abstract}

Keywords: Snake venom toxin multi-gene family; snake venom metalloproteinase; genomic organization of SVMP genes; PII-SVMP; PI-SVMP; gene duplication; intronic retroelements; intronization

\section{Introduction}

The ADAM (A Disintegrin-like And Metalloproteinase) family of transmembrane type 1 proteins belongs to the MEROP database M12 family of $\mathrm{Zn}^{2+}$-dependent metalloendopeptidases [1] and PFAM family PF01421 [2]. Members of the ADAM family play important roles in cell signaling and in regulating cell-cell and cell-matrix interactions [3,4]. The ADAM family comprises ancient proteins whose origin extends back $>750$ My $[5,6]$. To date, close to 40 ADAM genes have been identified in vertebrate and invertebrate bilaterian animals, both in deuterostomes, from the basal chordate, Ciona intestinalis, to higher vertebrates, and in protostome, such as arthropods, nematodes, platyhelminths, rotifers, molluscs, and annelids. The evolutionary history of vertebrate ADAM genes is punctuated by gene duplication and retroposition events $[7,8]$, followed by neo- or subfunctionalization [7]. Gene duplications are an essential source of genetic novelty that can lead to evolutionary innovation if the new function has no deleterious effects to its host organism or provides selective advantages. For example, in mammalian species, including marsupials and monotremes, except the platypus, ADAM28, ADAMDEC1 (decysin, a soluble ADAM-like protein), and ADAM7 form a cluster, likely as a result of tandem duplication of ADAM28 [9]. Instead, in most non-mammalian vertebrate genomes investigated, including those of aves, reptiles, and fishes, a single ADAM28 locus is present in this region $[7,10]$. The data suggest that ADAM7 and ADAMDEC1 were duplicated from 
ADAM28, probably only in mammals [7]. On the other hand, as described below in more detail, it is thought that ADAM28 played a starring role in the emergence of toxic metalloproteinases in the superfamily Colubroidea of Caenophidian snakes (viperids, elapids, and colubrids).

The concept that gene duplication plays a major role in evolution has been around for over a century [11]. In his classic and influential book "Evolution by Gene Duplication" [12] Susumo Ohno argued that gene duplication is the most important evolutionary force since the emergence of the universal common ancestor. Common sources of gene duplications include ectopic homologous recombination, retrotransposition event, aneuploidy, polyploidy, and replication slippage [13]. Duplication creates genetic redundancy, where the second copy of the gene is often free from selective pressure. Thus, over generations of the organism, duplicate genes accumulate mutations faster than a functional single-copy gene, making it possible for one of the two copies to develop a new and different function. Duplicated genes may switch their transcription to other tissues by localizing closely to, and utilizing the regulatory elements of, a neighboring gene [14-16]. Examples of this are (i) the formation of toxin gene families during the evolution of the venom system of advanced snakes by co-option, multiplication, and weaponization in the venom gland of paralogs of genes encoding for normal body proteins [17-20], and (ii) the finding of 309 distinct widow spider genes exhibiting venom gland biased expression [21], suggesting that the switching of genes to venom gland expression in numerous unrelated gene families has been a dominant mode of evolution [21-23].

Because of its functional importance for prey capture, predator defense, and competitor deterrence, venom represented a key innovation that has underpinned the explosive radiation of toxicoferan reptiles in the Late Jurassic period of the Mesozoic era, 150 million years before present (MYBP) [24-28]. Toxicofera [18] (Greek for "those who bear toxins") is the term coined for the clade of squamate reptiles that includes the Serpentes (snakes), Anguimorpha (monitor lizards, gila monster, and alligator lizards) ,and Iguania (iguanas, agamas, and chameleons) lizards. One of the founding families of advanced snake venom comprises the $\mathrm{Zn}^{2+}$-dependent metalloendopeptidases (SVMPs) [17-19,29-32]. SVMPs are key enzymes contributing to toxicity of vipers and pitvipers venoms. Hemorrhage is one of the most significant effects in envenomings induced by viperid and crotalid snakebites. Damage to the microvasculature, induced by SVMPs, is the main event responsible for this effect. In addition to hemorrhagic activity, members of the SVMP family also have fibrin(ogen)olytic activity, act as prothrombin activators, activate blood coagulation factor $\mathrm{X}$, possess apoptotic activity, inhibit platelet aggregation, are proinflammatory, and inactivate blood serine proteinase inhibitors [33-36].

The closest non-venom ancestors of SVMPs was likely an ADAM28 precursor gene [37]. The origin of SVMPs has been inferred to have occurred following the split of the Pareatidae from the remaining Caenophidians, approximately 60 MYBP around the Cretaceous-Paleocene boundary of the Cenozoic Era $[18,19,29,31,38]$. SVMPs are found in the venoms of all advanced snakes and are classified into different classes depending upon their domain structure [39-41]. The ancestral multidomain PIII form, which is found in all snake venoms, derives from the extracellular region (metalloproteinase domain with disintegrin-like and cysteine-rich domains at the $C$-terminus) of a duplicated ADAM28 precursor gene that lost the $C$-terminal epidermal-growth-factor (EGF-)-like, transmembrane, and cytoplasmic domains $[31,32,41-43]$. On the other hand, the derived PII-SVMPs, comprising the metalloproteinase and $C$-terminal disintegrin domain, have been only found in venoms of vipers and rattlesnakes (Viperidae). This strongly suggests that they emerged, subsequently to the separation of Viperidae and Elapidae, 37 million years ago, in the Eocene epoch of the Cenozoic era, but before the separation of the Viperidae subfamilies Viperinae and Crotalinae 12-20 MYBP, from a duplicated PIII-SVMP gene that lost its cysteine-rich domain (see Figures 1 and 8 in [43] and Figure 18.1 in [44]). The disintegrin domain has been lost from the PII-SVMP structure on multiple occasions, resulting in the formation of the PI class of SVMPs [45] made only by the catalytic $\mathrm{Zn}^{2+}$-metalloproteinase domain [39-41].

Details on the mechanisms of co-option and the molecular events underlying the transformation of an ADAM28 precursor gene copy into the SVMP multi-gene family of extant snake venoms 
remain elusive. In previous works, we described a family of RPTLN genes that exhibit a broad and reptile-specific distribution, for which we hypothesize may have played a key role in the recruitment and restricted expression of SVMP genes in the venom gland of Caenophidian snakes [46]. We have also reported the genomic organization of Echis ocellatus PIII-SVMP gene EOC00089, and compared it to those of its closest orthologs from Homo sapiens and the lizard, Anolis carolinensis [47]. Now, we fit two new pieces in the puzzle: the genomic structures of E. ocellatus PII-(EOC00006-like) and PI-(EOC00028-like) SVMP genes. Insights into post-duplication events gained from the structural comparison of the three classes of SVMP genes are discussed.

\section{Results and Discussion}

\subsection{The Genomic Structure of Pre-Pro EOC00006-Like PII-SVMP and Pre-Pro EOC00028-Like Genes}

Genomic sequences encoding full-length pre-pro EOC00006-like PII-SVMP (17828 nt) [KX219964] (Figure A1) and EOC00028-like PI-SVMP (21605 nt) [KX219965] (Figure A2) genes were assembled from overlapping PCR-amplified fragments (Appendix A, Figures A1 and A2). The pre-pro PII-SVMP gene consists of 15 exons interrupted by 14 introns (Figure 1A), whereas the pre-pro PI-SVMP gene contains 13 exons and 12 introns (Figure 1B).
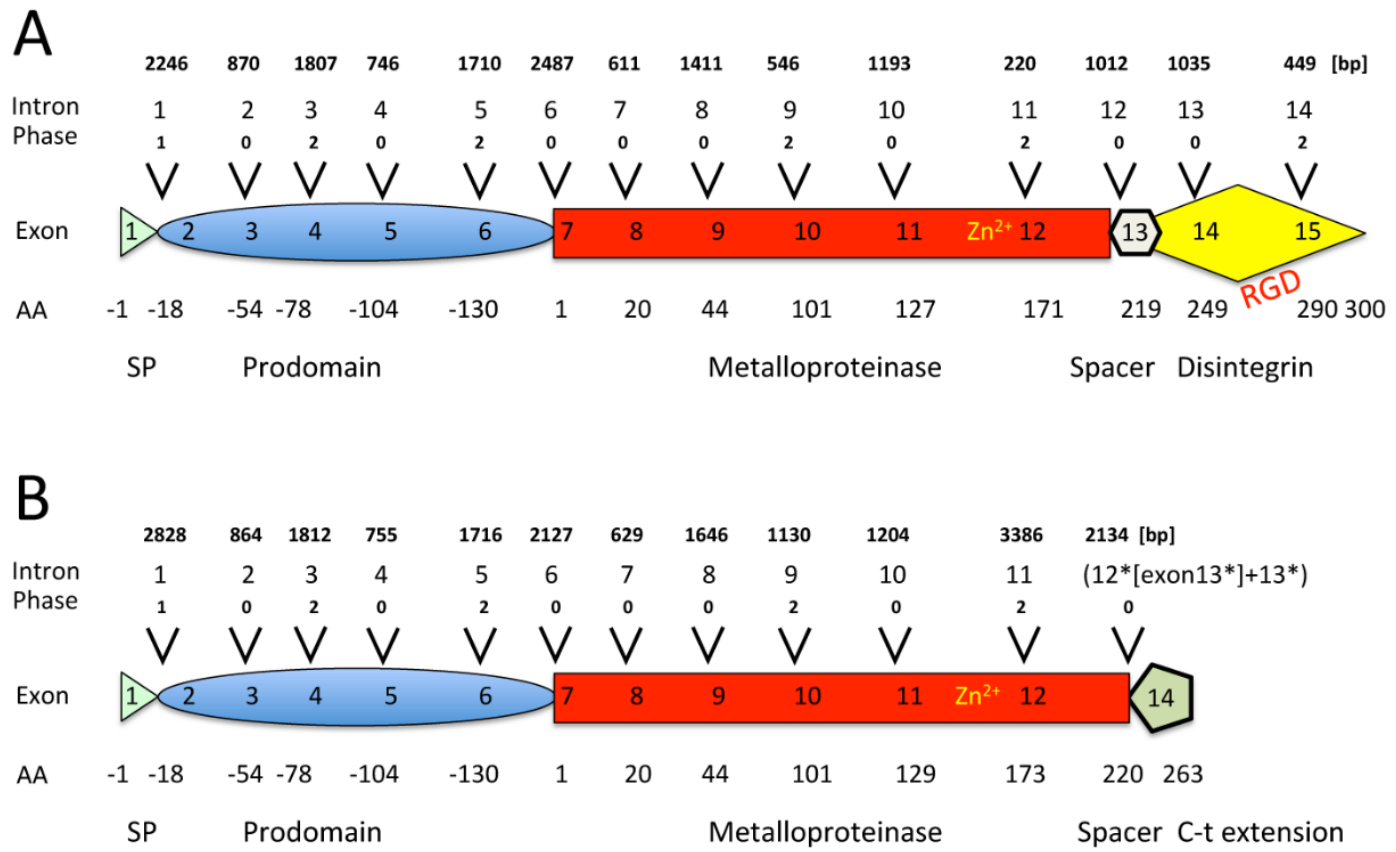

Figure 1. Scheme of the genomic organization of pre-pro EOC00006-like PII-SVMP (A) and pre-pro EOC00028-like PI-SVMP (B) genes. The distribution, phase, and size of the 14 (PII) and 12 (PI) introns and the boundaries of the protein-coding regions are highlighted. SP, signal peptide. Homologous exons and introns have identical numbering. Intron 12 of the PI-SVMP gene corresponds to the fusion of the genomic segment spanning intron $12^{*}$-exon13*-intron13*. Mature PII- and PI-SVMP amino acid sequences span 299 and 263 amino acid residues, respectively. $\mathrm{Zn}^{2+}$, relative location of the catalytic $\mathrm{Zn}^{2+}$-binding environment; RGD, integrin-binding arginine-glycine-aspartic acid tripeptide motif.

The translated 494 (PII) and 457 (PI) pre-pro-SVMP amino acid sequences exhibit identical distribution and features (in terms of codon location and phase) for their first 11 introns and 12 exons, which code for the signal peptide (SP), prodomain (PD), metalloproteinase (MP) domain, and the short tetrapeptide (ELLQ) "spacer" sequence (Appendix A, Figures A1 and A2). These 413 (PII)/414 (PI) amino acid sequences show $85 \%$ identity, strongly suggesting that both SVMPs have a shared ancestry. It is also worth noting that the protein-coding positions interrupted by each of the introns 
of the PII- and PI-SVMP genes are entirely conserved in Anolis carolinensis [XP_008118058] (and also in human [NG_029394]) ADAM28 gene. Introns are inserted after or between secondary structure elements, supporting the "introns-added-late" model, which proposes that during the evolution of the eukaryotic branch, introns were added at the boundaries of structural modules coded for by ancestral continuous genes [48]. In addition, as will be analyzed in detail below, pairwise alignment of topologically equivalent PII- and PI-SVMP introns show that homologous intronic nucleic acid sequences share 88\%-99\% identity (Figure 2). This clearly indicates that EOC00006-like PII-SVMP and EOC00028-like PI-SVMP represent paralog genes.

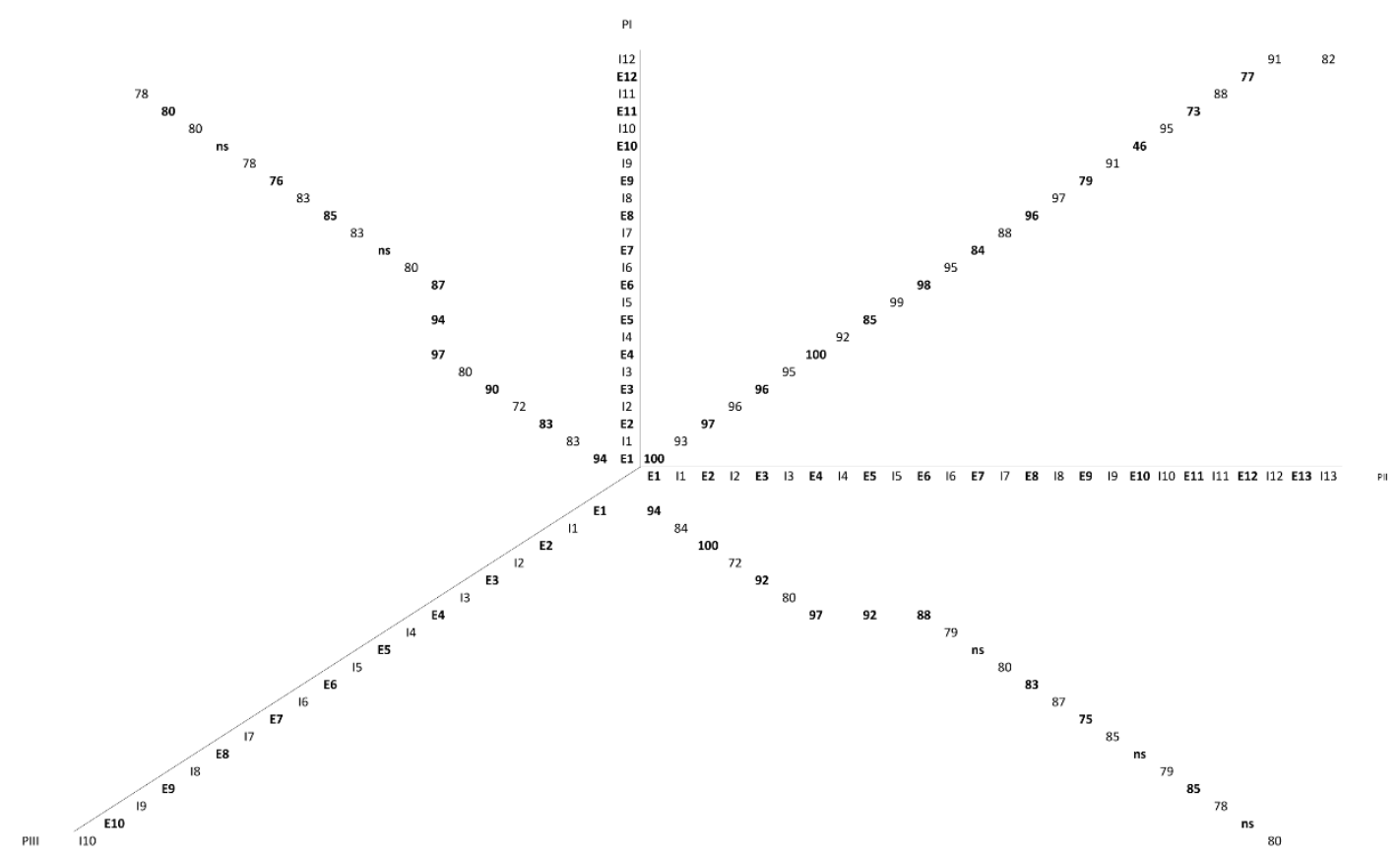

Figure 2. Pairwise comparisons of the sequence identities between the exonic and intronic nucleic acid sequences of pre-pro EOC00089-like PIII-SVMP, EOC00006-like PII-SVMP, and EOC00028-like PI-SVMP genes.

New genes can arise through four mechanisms: gene duplication, retroposition, horizontal gene transfer, and de novo origination from non-coding sequences [49]. Available evidence strongly suggests that gene duplication has played a pivotal role in the origin of venom multi-gene families [20-23,50,51]. Although the fate of many new genes may be to lose their function and become pseudogenes, some can be fixed through evolution of redundancy, subfunctionalization, or neofunctionlization. Several models have been proposed to explain functional divergence following venom toxin gene duplications [52-55]. However, this issue remains controversial and is the subject of vivid debates. The family portrait of SVMPs shows a complicated picture. SVMPs belong to different "generations", that in the canonical model for the evolutionary expansion of this multi-gene family are hierarchically related, being PIII-SVMPs the most ancient and the PII- and PI- SVMPs the succesively most recently derived family members [31,32,42]. However, due to the limited genomic information available, this model can be confounded by high rates of protein amino acid sequence divergence [56], and the occurrence of alternative routes (e.g., PIII > PI) can not be presently ruled out. The only other full-length viperid SVMP gene sequenced to date is E. ocellatus EOC00089-like PIII-SVMP [47] [KX219963]. The ORF encoding the pre-pro-metalloproteinase domains of this gene exhibits $63 \%$ amino acid sequence identity with the homologous coding regions of the PII- and PI-SVMPs here reported, and $72 \%-83 \%$ nucleotide sequence identity between topologically equivalent PIII-, PII-, and PI-SVMP introns (Figure 2). Although these figures clearly point to a common origin, it is not possible to infer whether they belong to the same or to a different PIII > PII > PI hierarchical lineage. Nonetheless, the 
fact that the PIII-SVMP gene has lost introns 5 and 6 (ADAM28 numbering), with the consequence that exons 4, 5, and 6 have merged into a single exon, suggests that either these events occurred after the duplication that gave rise to the PII-SVMP ancestor, or that the PIII-SVMP EOC00089-like gene does not lay in the direct line of descent of the EOC00006-like PII-SVMP and EOC00028-like PI-SVMP genes. Refinement of the family tree of the multi-gene family of E. ocellatus SVMPs will surely emerge from future comparative genomic analysis of the carpet viper and other viperid species.

\subsection{Role of Introns in the Evolution of the SVMP Multi-Gene Family}

Since their discovery in 1977 [57,58], introns have been the subject of considerable debate. It is now generally accepted that introns represent more than merely junk DNA that must be pruned from pre-mRNAs to yield mature, functional mRNAs prior to their translation. Mounting evidence indicates that while introns do not encode protein products, they play essential roles in a wide range of gene expression regulatory functions such as non-sense mediated decay [59], mRNA export [60], and regulation of the amount of recombination between the flanking exons [61], or they serve as locations for nonhomologous recombination that would allow for exon shuffling [62,63]. As discussed below, most of the structural divergence between the EOC00006-like PII-SVMP and EOC00028-like PI-SVMP genes is due to the different size of their topologically equivalent eleven (1-11) introns (Supplementary Figure S1). The role of introns in the evolution of snake venom gene families remains elusive. However, in other biological systems, i.e., Arabidopsis and Drosophila, intron features, such as sequence and length, have been shown to function in maintaining pre-mRNA secondary structure, thus influencing temporal and spatial patterns of gene expression by modulating transcription efficiency and splicing accuracy [64-67].

Most PII- and PI-SVMP introns belong to phase 0, followed by phase 2; and, in both genes, only intron 1, separating the monoexonic signal peptide from the start of the prodomain, is a phase 1 intron (Figure 1). Analysis of the exon-intron structures of a large number of human genes has revealed a statistically highly significant enrichment of phase 1 introns flanking signal peptide cleavage sites [68]. Phase 1 introns most frequently split the four GGN codons encoding glycine. A plausible explanation for the correlation between signal peptide domains and the intron phase is that the base preferences of proto-splice sites $[69,70]$ mirrors the amino acid preference for glycine in the signal peptidase consensus cleavage site [71].

The signal peptide is the most conserved structural element between pre-pro EOC00006-like PII-SVMP and EOC00028-like PI-SVMP is (Figure 2). In both genes, it is encoded by identical exon 1 amino acid sequences (Figures A1 and A2), which is also highly conserved in present-day SVMPs [46]. These findings support the view that co-option of this signal peptide may have played a role in the restricted expression of SVMP genes in the venom gland of Caenophidian snakes, some 60-50 Mya [46].

Nucleotide sequence comparison of the topologically equivalent introns of the E. ocellatus PII- and PI-SVMPs (Supplementary Figure S1) provide insights into the events underlying the conversion of a PII-SVMP into a PI-SVMP gene. In this regard, some introns differ in the number and location of intronic retroelements (Table 1). Thus, insertions in introns PI-SVMP 1 and 9 introduced complete and truncated SINE/Sauria elements in positions 1764-2101 (Figure S1, panel A) and 321-502 (Figure S1, panel I), respectively. The inserted nucleic acid sequence in intron 9 retains the GT-AG splicing sites, indicating that this insertion event created a twintron, an intron within an intron. PII-SVMP intron 6 (Figure S1, panel F) and PI-SVMP introns 11 (Figure S1, panel K) and 12 (Figure S1, panel L) are also twintrons. Compared to its topologically equivalent PII-SVMP intron, a large insertion in intron 11 of the EOC00028-like PI-SVMP gene replaced the first 66 nucleotides for a longer stretch of 3281 nucleotides; region 2461-2561 of the inserted nucleic acid sequence is $97 \%$ identical to Hyla tsinlingensis Hts-35 [KP204922], a microsatellite sequence that is also partly present in intron 61 of Podarcis reelin (RELN) genes [GU181006-13] (positions 554-623) [72]. Microsatellites are simple nucleotide sequence repeats (SSR) ranging in length from two to five base pairs that are tandemly repeated, typically 5-50 times (reviewed in [73]). These non-coding elements are abundant in major 
lineages of vertebrates. Mammalian, fish, and squamate reptile genomes appear to be relatively microsatellite rich [74]. However, besides Hts35, RepeatMasker only identified few SSR tracks in introns $1(5 \times$ GTTT; $28 \times$ TC) and $2(13 \times$ ATTT; $4 \times$ TAA) of the PII-SVMP gene (Figure A1), and introns $1(11 \times$ GTTT; $21 \times$ AG) and $2(9 \times$ GTTT; $4 \times$ TAA) of the PI-SVMP gene (Figure A2).

Table 1. Comparison of type and location of retroelements identified in introns of E. ocellatus PII-SVMP EOC00006-like and PI-SVMP EOC00028-like genes.

\begin{tabular}{ccc}
\hline \multirow{2}{*}{ Intron } & PII-SVMP & PI-SVMP \\
\cline { 2 - 3 } & \multicolumn{1}{c}{ Inserted Retroelement } \\
\hline \multirow{2}{*}{1} & SINE/Sauria & 2 SINE/Sauria, LTR/ERV1, \\
3 & LINE/L2/CR1 & DNA/hAT-Ac \\
5 & LINE/L2/CR1 & LINE/L2/CR1 \\
6 & SINE/Sauria & - \\
8 & LINE/L2/CR1 & - \\
9 & - & SINE/Sauria \\
10 & DNA transposon & DNA transposon \\
\hline
\end{tabular}

Growing evidence supports that repetitive intronic elements, such as the long interspersed elements (LINEs) and the short interspersed elements (SINEs) contained in several introns of both PII- and PI-SVMP genes (Table 1) can influence genome stability and gene expression (reviewed in [75]). Thus, these interspersed repeats may alter genome recombination structure and rates, through a number of mechanisms, including replication slippage and unequal crossover [76,77], potentially impacting regulation of gene expression [78], recombination events leading to tandem duplication of segments of the genome [79,80], gene conversion [81], and chromosomal organization [79]. Moreover, the insertion of interspersed repeats into a new genomic position may introduce promoter or enhancer sequence motifs for transcription of nearby genes $[82,83]$, and alternative splicing sites or polyadenylation sites [84], thereby resulting in a change of overall level of gene expression. Interspersed repetitive elements have also played an important role in expanding the repertoire of transcription factor binding sites in eukaryotic genomes [85]. However, whether these elements have contributed to the genomic context that facilitated the evolution and radiation of venom loci in snakes deserves future detailed comparative genomic studies.

\subsection{A Fusion Event Led to the Conversion of a PII(e/d)-Type SVMP into EOC00028-like PI-SVMP}

PI-SVMP intron 12 is a twintron resulting from the fusion of the genomic region spanning ancestral introns $12^{*}$ and $13^{*}$ and exon $13^{*}$ (homologous to identical numbered elements in the genomic structure PII) (Figures 1 and 3A). Splicing site mutations affecting both the $3^{\prime}$ splice AG acceptor site of intron $12^{*}$ and the $5^{\prime}$ splice GT donor site of intron $13^{*}$ led to the retention, and subsequent intronization, of exon $13^{*}$ within a fused $\left(12^{*}+13^{*}\right)$ twintron (Figure $\left.3 \mathrm{~A}\right)$. Intronization of exon-coding nucleic acid sequences has been proposed as a major contributor to intron creation [86]. Intron $13^{*}$ encoded part of the $N$-terminal region of a disintegrin domain, most likely, as discussed below, an eventual subunit of dimeric disintegrin. In addition to the disruption of the structural integrity of the disintegrin domain, a stop codon after exon 14 removed intron 14 and exon 15 from the PII(e/d)-type SVMP (Fox \& Serrano's nomenclature [40]) precursor gene structure, thereby completing the conversion of the PII-SVMP into present EOC00028-like PI-SVMP gene (Figure 3A). 


\begin{tabular}{|c|c|c|c|c|c|c|c|c|}
\hline $\begin{array}{r}\text { PII-SVIVIP } \\
\text { Exon } 12\end{array}$ & & Intron 12 & & $\begin{array}{c}\rightarrow \text { Disintegri } \\
\text { Exon } 13\end{array}$ & & Intron 13 & & Exon 14 \\
\hline .... TPVSENELLQ & GT & $1012 \mathrm{nt}$ & $A G$ & NSVNPC ... DNCK & GT & $1035 \mathrm{nt}$ & AG & FLKEG ... PMEW \\
\hline
\end{tabular}

\begin{tabular}{|c|c|c|}
\hline Exon 12 & Intron 12 (2134 nt) [Intron $12^{*}+$ Exon $13^{*}+$ Intron $\left.13^{*}\right]$ & Exon 14 \\
\hline .... TPVSENELLQ & at ${ }^{* Y D P C C} \ldots$ LVYF* aC & FLRAR ... WNDLQ \\
\hline
\end{tabular}

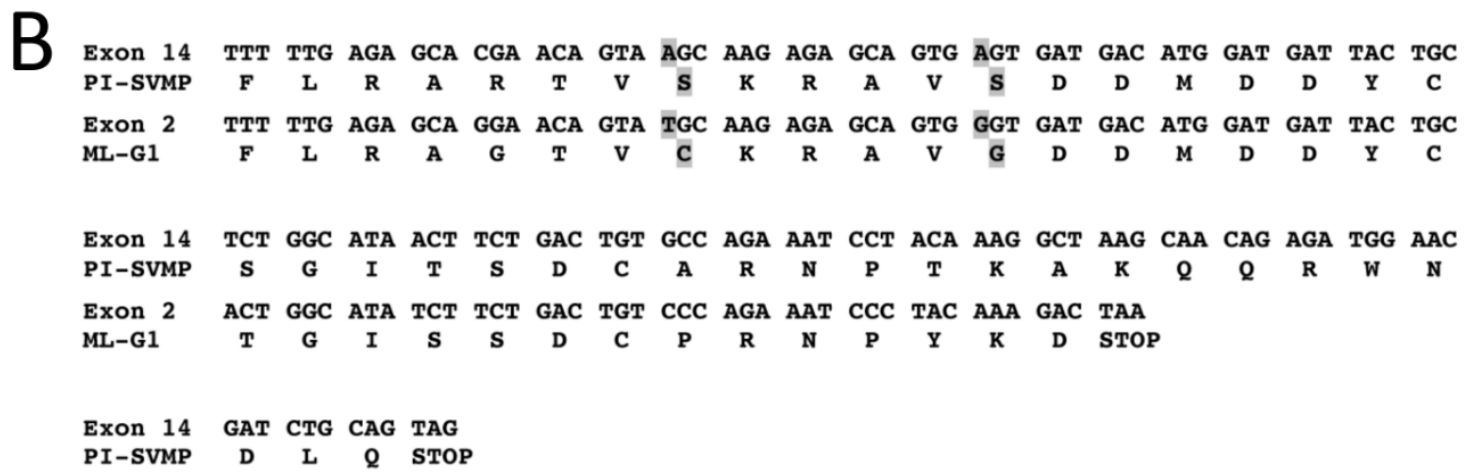

Figure 3. Panel A, cartoon comparing the $3^{\prime}$ regions of the PII-SVMP and PI-SVMP genes and highlighting the processes (intronization of ancestral exon $13^{*}$ inside twintron 12 resulting from the fusion of introns $12^{*}$ and $13^{*}$, and creation of a stop codon after exon 14) that destroyed the integrity of the disintegrin domain, converting an ancestral PII(e/d)-type SVMP into extant EOC00028-like PI-SVMP. Panel B, alignment of the amino acid sequences encoded by exon 14 of EOC00028-like PI-SVMP and exon 2 of the dimeric disintegrin subunit ML-G1 [AM261811] [87]. Degeneration of PI-SVMP's conserved functional and structural amino acid residues in dimeric disintegrins are highlighted in boldface and grey background.

Region 1013-2134 of PI-SVMP intron 12 exhibits 91\% nucleotide sequence identity with range 14 to 1135 of Macrovipera lebetina gene encoding part of exon 1 and full-length intron 1 of the VGD-containing dimeric disintegrin subunit precursor, ML-G1 [AM261811] [87]. PI-SVMP exon 14 (mature protein amino acid residues 221-263, Figure A2) exhibits strong homology (79\% identity) to exon 2 of the same VGD-bearing dimeric disintegrin subunit. The PI-SVMP exon 14 shows the consequences of genetic drift (Figure 3B): the conseved $\alpha_{5} \beta_{1}$ integrin-inhibitory VGD tripeptide motif [44] of the PII-SVMP precursor gene has been replaced by a V $\underline{S D}$ motif (generated by a G > A mutation: GTG $\underline{A} G T$ GAT > GTG GGT GAT), and the absolutely conserved tenth cysteine residue of dimeric disintegrin subunits has degenerated (TGC) to a serine residue (AGC) (Figure 3B).

\section{Concluding Remarks and Perspectives}

The event that gave birth to the family of SVMPs was the generation of a STOP codon at the $3^{\prime}$ end of exon 16 of a duplicated ADAM28 gene (Figure 4). This mutation produced an ORF truncated at the $N$-terminal part of the EGF-like domain, which encoded a precursor of an ancestral PIII-SVMP lacking this domain and the C-terminal membrane anchoring and cytoplasmic polypeptides (Figure 4). On the other hand, our results comparing the available genomic structures of SVMP genes, e.g., EOC00089-like PIII-SVMP [47] [KX219963], EOC00006-like PII-SVMP [KX219964], and EOC00028-like PI-SVMP [KX219965] (this work), suggest that the evolutionary history of SVMPs is marked with events of insertions and deletions of intronic regions. This scenario points to introns as key players in 
the formation of the multi-locus SVMP gene multifamily. Thus, comparison of the genomic structures of EOC00089-like PIII-SVMP and EOC00006-like PII-SVMP (Figure 5) indicates that replacement of the PIII-specific cysteine-rich domain by a non-homologous region encoding intron 14-exon 15 followed by a STOP codon may represent a step in the conversion of a PIII-SVMP into a PII-SVMP.

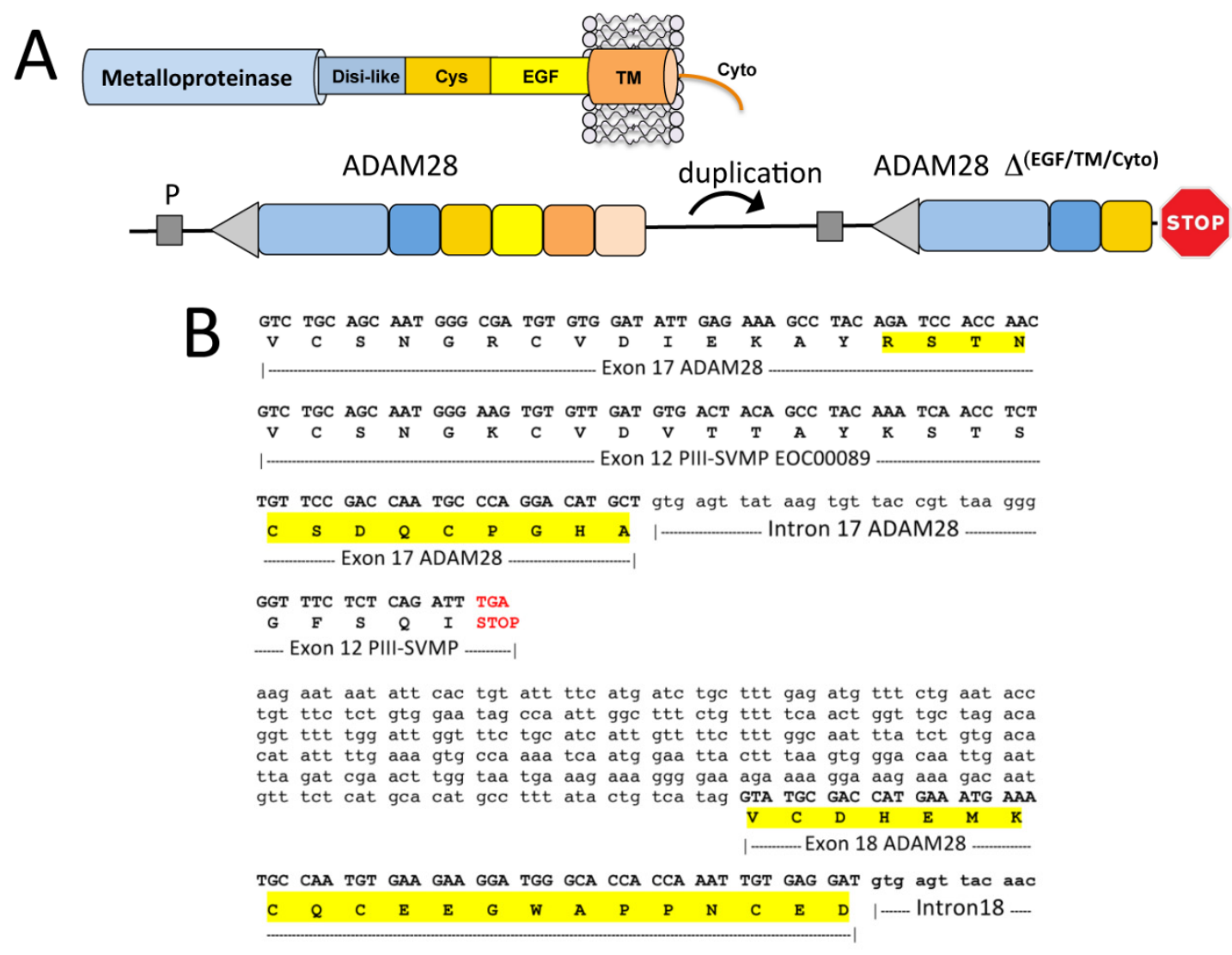

Figure 4. Comparison of the genomic region encompassing exons 17 through 18 of Anolis carolinensis ADAM28 [XP_003226913] and the homologous amino acid sequence of E. ocellatus SVMP EOC00089 [ADW54351], highlighting the STOP codon after exon 12 of the latter generating a C-terminally truncated molecule, which eventually gave rise to the ancestor of the PIII-SVMPs.

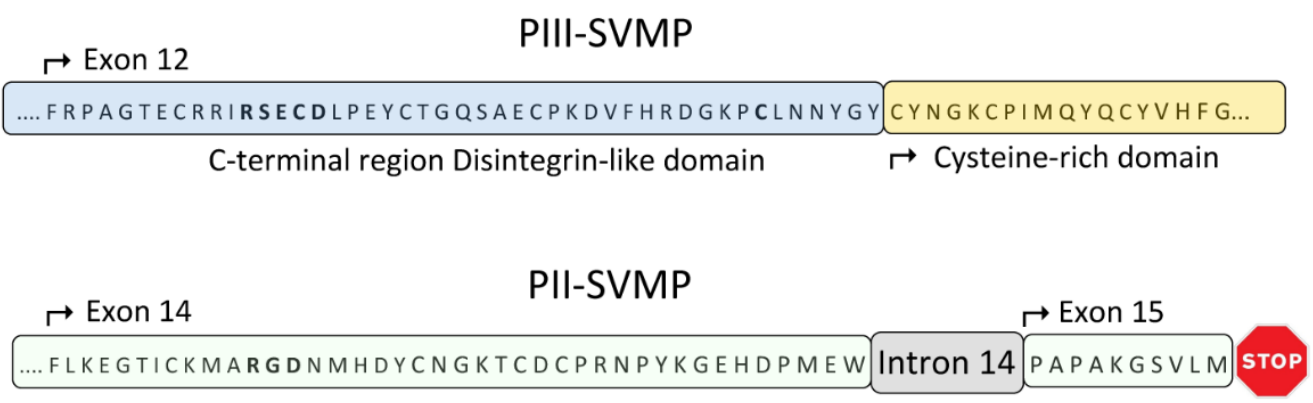

C-terminal region of the gene encoding the short disintegrin ocellatusin [KX219964]

Figure 5. Comparison of the genomic region encoding the C-terminal domains of E. ocellatus EOC00089-like PIII-SVMP and EOC00006-like PII-SVMP, suggesting that $3^{\prime}$ genomic remodeling represents a seminal step in the generation of PII-SVMPs.

This view is consistent with structural evidence suggesting that the loss of the cysteine-rich domain represents an early seminal event that facilitated the formation of PII class SVMPs [43]. 
The PII subfamily of SVMPs is characterized by the diversity of disintegrin domains exhibited by different family members $[39,40]$, ranging from the more ancestral long disintegrin domains ( $~ 84$ amino-acid-residue polypeptide cross-linked by 7 disulfide linkages) to the more recently evolved short disintegrin (41-51 amino-acid-residues crosslinked by 4 disulfide bonds) [42]; for a scheme of the evolutionary path of the disintegrin domains, see Figure 1 in [43]. EOC00006-like is an example of a PII-SVMP with short disintegrin domain. Given the structural diversity of PII-SVMPs, genomic sequences from the different members of the subfamily are required for a more accurate glimpse of the genomic mechanisms operating in the generation and subsequent diversification of PII-SVMPs.

Comparison of the EOC00006-like PII-SVMP and EOC00028-like PI-SVMP gene structures also points to genomic remodeling of the $3^{\prime}$ region of a PII(e/d)-type SVMP precursor gene $[39,40]$ as the EOC00028-like PI-SVMP gene generator mechanism. The PII > PI conversion involved the generation of twintron 12 (by fusion of introns $12^{*}$ and $13^{*}$ ) and the loss, by intronization, of exon $13^{*}$, thereby destroying the consistency of the region coding for the disintegrin domain. This elaborated mechanism indicates that the structural diversification of SVMPs is not due to a random mutation generating a STOP codon before the disintegrin domain, but follows a well orchestrated sequence of events imprinted in the genome of snake species sometime after the split of Viperidae and Elapidae, 37 million years ago, but before the separation of the Viperidae subfamilies Viperinae and Crotalinae 12-20 MYBP. The mechanisms underlying loss or gain of spliceosomal introns are still poorly understood. The most widely accepted hypothesis is that intron insertion may occur via a process similar to group II intron retrotransposition [88,89]. According to this view, the spliceosomal components remain transiently associated with a recently excised intron and then attach at a potential splice site of a non-homologous pre-mRNA, where they catalyze the reverse reaction [90,91]. The modified pre-mRNA is reverse-transcribed and the resulting cDNA participates in a recombination with its parent gene, thereby inserting a novel intron into the target gene [90-93]. An attractive feature of this mechanism is that it ensures that the inserted nucleic acid sequence has the full complement of intron signature sequences required for efficient splicing [94].

Studies of multi-gene protein families are crucial for understanding the role of gene duplication and genomic exon-intron organization in generating protein diversity. For example, full-length genomic sequences of Crotalinae group II PLA 2 isogenes from $P$. flavoviridis (Tokunoshima and Amami-Oshima islands, Japan) [95], and T. gramineus (Taiwan) [96] have been reported. All these genes exhibit four coding regions and conserved exon-intron structures spanning about $1.9 \mathrm{~kb}$. A cluster of five tandemly arranged PLA 2 genes have been located in a $25 \mathrm{~kb} 3^{\prime}$ segment of a $31 \mathrm{~kb}$ fragment of the Amami-Oshima P. flavoviridis genome [97], which in addition harbors a PLA 2 pseudogene in its $6 \mathrm{~kb} 5^{\prime}$ region [98]. Genomic sequence comparisons between the pancreatic PLA 2 gene of P. elegans, group IB pancreatic PLA 2 gene of L. semifasciata, and the L. semifasciata group IA venom PLA 2 gene, suggest that Crotalinae group II venom $\mathrm{PLA}_{2}$ genes emerged before the divergence of Elapinae and Crotalinae, whereas groups of IB and IA PLA 2 genes appeared after Elapinae was established as a taxonomic lineage [99].

Duplicated structures found in eukaryotic genomes may result from complex interplays between different mechanisms [100]. Mitotic and meiotic non-allelic homologous recombination (NAHR) events, resolved as unequal crossing-over, have been traditionally invoked to account for segmental duplications within genomes [101,102]. Duplicated regions can be organized as direct tandems (e.g., the cluster of tandem snake venom $\mathrm{PLA}_{2}$ genes), but also be separated by hundreds of kb [100]. Our present and previous work [47] inaugurate a line of research that will allow the depiction of a more precise characterization of the genomic context in which the SVMP multi-gene family has emerged. This goal demands populating the current databases with genomic sequences of genes representing the different members of the SVMPs. Although the variety of structural forms comprising the PII family may be considered a challenge for this purpose, this circumstance can be also regarded as a valuable opportunity for the step-by-step description of the molecular pathways that led to the formation of this multi-gene family. Without a doubt, ongoing Viperidae snake genome sequencing projects will mark 
the beginning of comparative snake genomics, and will be key to revealing not only the topology and copy number of the genes encoding SVMPs, but also to provide decisive information to reconstruct the evolutionary history of this multilocus gene family.

\section{Materials and Methods}

\subsection{Genomic DNA}

Genomic DNA was extracted from the fresh liver of E. ocellatus (Kaltungo, Nigeria) maintained at the herpetarium of the Liverpool School of Tropical Medicine. Echis ocellatus liver was ground to a fine powder under liquid nitrogen and the genomic DNA extracted using a Roche DNA isolation kit for cells and tissue containing SDS ( $2 \%$ final concentration) and proteinase $\mathrm{K}(400 \mu \mathrm{g} / \mathrm{mL}$ final concentration). The homogenates were incubated at $55^{\circ} \mathrm{C}$ overnight. Thereafter, $300 \mu \mathrm{L}$ of $6 \mathrm{M} \mathrm{NaCl}$ ( $\mathrm{NaCl}$-saturated $\mathrm{H}_{2} \mathrm{O}$ ) was added to each sample, and the mixture was vortexed for $30 \mathrm{~s}$ at maximum speed and centrifuged for $30 \mathrm{~min}$ at $10,000 \mathrm{~g}$. An equal volume of isopropanol was added to each supernatant, and the sample mixed, incubated at $-20^{\circ} \mathrm{C}$ for $1 \mathrm{~h}$, and centrifuged for $20 \mathrm{~min}$ at $4{ }^{\circ} \mathrm{C}$ and $10,000 \mathrm{~g}$. The resulting pellets were washed with $70 \%$ ethanol, dried, and, finally, resuspended in 300-500 $\mu \mathrm{L}$ sterile distilled $\mathrm{H}_{2} \mathrm{O}$.

\subsection{Strategy for PCR Amplification of Overlapping Genomic DNA Fragments}

For sequencing E. ocellatus genes encoding PII-SVMP EOC00006 [Q14FJ4] and PI-SVMP EOC00028 [Q2UXQ3] we employed a similar iterative process as described in [47]. Full-length cDNA-deduced amino acid sequences of disintegrin domains [103] and of the genomic organization of dimeric disintegrin domains [AM286800] [87] and PIII-SVMP EOC00089 [47] from the same species were used as templates to design primers for the PCR-amplification of protein-specific genomic sequences (Table 2).

PI-SVMP stretch ${ }^{72}$ AREILNS.....QRWNDLQ ${ }^{263}$ was amplified on an Eppendorf Mastercycle ${ }^{\circledR}$ epgradient $\mathrm{S}$ instrument in a $50 \mu \mathrm{L}$ reaction mixture containing $17.5 \mu \mathrm{L}$ of $\mathrm{H}_{2} \mathrm{O}, 25 \mu \mathrm{L}$ Master-Mix (Thermo Scientific, Waltham, MA USA) including buffer, dNTPs, and Phusion High-Fidelity DNA polymerase, $2.5 \mu \mathrm{L}$ of each primer $(10 \mu \mathrm{M})$ Met1PIRv and Met5PIFw, $1.5 \mu \mathrm{L}$ of DMSO (100\%), and $1 \mu \mathrm{L}$ of genomic DNA $(50 \mathrm{ng} / \mu \mathrm{L})$. PCR conditions included an initial denaturation step at $98^{\circ} \mathrm{C}$ for $30 \mathrm{~s}$ followed by 35 cycles of denaturation $\left(20 \mathrm{~s}\right.$ at $\left.98^{\circ} \mathrm{C}\right)$, annealing $\left(15 \mathrm{~s}\right.$ at $\left.63^{\circ} \mathrm{C}\right)$, extension $(300 \mathrm{~s}$ at $72{ }^{\circ} \mathrm{C}$ ), and a final extension for $5 \mathrm{~min}$ at $72{ }^{\circ} \mathrm{C}$. All other PCR amplifications were carried out in the same thermocycler using iProof High Fidelity polymerase (BioRad, Hercules, CA, USA). The $50 \mu \mathrm{L}$ reaction mixture contained $10 \mu \mathrm{L}$ of $5 \times$ buffer, $1 \mu \mathrm{L}$ of $10 \mathrm{mM}$ (each) dNTPs, $2 \mu \mathrm{L}$ of $\mathrm{MgCl}_{2} 50 \mathrm{mM}$, $1.5 \mu \mathrm{L}$ of DMSO (100\%), $1 \mu \mathrm{L}$ of each Fw and Rv primer $(10 \mu \mathrm{M}), 1 \mu \mathrm{L}$ of genomic DNA $(50 \mathrm{ng} / \mu \mathrm{L})$, and $32.5 \mu \mathrm{L}$ of water. PCR conditions included an initial denaturation step at $98^{\circ} \mathrm{C}$ for $120 \mathrm{~s}$ followed by 35 cycles of denaturation $\left(10 \mathrm{~s}\right.$ at $\left.98^{\circ} \mathrm{C}\right)$, annealing $(15 \mathrm{~s}$ at the lower melting temperature of the primers), extension $\left(60 \mathrm{~s}\right.$ per $\mathrm{Kb}$ at $\left.72{ }^{\circ} \mathrm{C}\right)$, and a final extension for $5 \mathrm{~min}$ at $72{ }^{\circ} \mathrm{C}$.

\subsection{Purification and Cloning of PCR Products}

PCR-amplified DNA fragments were purified from agarose electrophoretic bands using the GENECLEAN Turbo kit (MP Biomedicals). The purified fragments were inserted into pJET_1.2 (Thermo Scientific, Waltham, MA USA) using phage T4 ligase and cloned into E. coli DH5 $\alpha$ by electroporation at $1700 \mathrm{~V}$. Transformed cells, resuspended in $200 \mu \mathrm{L}$ LB medium, were incubated at $37^{\circ} \mathrm{C}$ for $1 \mathrm{~h}$, and were subsequently plated on LB agar/ampicilline to select positive clones. The presence of the inserted DNA fragments was verified by PCR amplification or digestion of the expression vector with the restriction enzyme Bgl II. The inserted DNA fragments were sequenced in-house on an Applied Biosystems model 377 DNA sequencing system (Foster City, CA, USA) using pJETFw and pJETRv primers. 
Table 2. Forward $(\mathrm{Fw})$ and reverse (Rv) primers used to PCR-amplify genomic DNA stretches from E. ocellatus PII-SVMP EOC00006-like (right) and PI-SVMP EOC00028-like (left) genes.

\begin{tabular}{|c|c|c|c|}
\hline Primer & DNA sequence & Primer & DNA sequence \\
\hline Sp35_Eo Fw & ATGATCCAAGTTCTCTTGGTAACTATATGCTTAGC & 5' PS-Disi Fw & ATGATCCAAGTTCTCTTGG \\
\hline Met14PI Fw & CTATATGCTTAGCAGTTTTTCCATATC & Intr4 Fw & ATGACACTGACCTCTAGAGTTGG \\
\hline Intr1F1PI Fw & CTAGTCATTCCGGCCATATGAC & IntrB9_4-2 Fw & AAGCTTGCTTGCTAGTAGGTGG \\
\hline Intr2F1PI Fw & ATCAGTCTGAGAGGATGCATTTCC & Intr4 Rv & TGGACATTGTATGGTCACCTG \\
\hline Intr3F1PI Fw & GTGACCATGCAATGTCCATATG & Prodom 3 Fw & GGAGCTTTTAAGCAGCCAGAG \\
\hline Met15PI Fw & GTTGCCTGTAGGAGCTGTTAAG & Prodom $3 \mathrm{Rv}$ & CTCTGGCTGCTTAAAAGCTCC \\
\hline Prodom 2 Fw & GACGCTGTGCAATATGAATTTG & Prodom $2 \mathrm{Fw}$ & GACGCTGTGCAATATGAATTTG \\
\hline Prodom 2 Rv & CAAATTCATATTGCACAGCGTC & Prodom 2 Rv & CAAATTCATATTGCACAGCGTC \\
\hline Intr3 Rv & GCACCAACTCTGTATCTCAGTC & Intr3 Fw & CACAGGTAAATAAGCCACAAACACC \\
\hline Pro2 Fw & CAGTGAGACTCATTATTCCСCTGATGGCAG & Intr3 Rv & GCACCAACTCTGTATCTCAGTC \\
\hline Pro3 Rv & CTGCCATCAGGGGAATAATGAGTCTCACTG & Pro2-SVMP_Fw & $\begin{array}{l}\text { CAGAAGATTACAGTGAGACTCATTA } \\
\text { TTCCCWGATGG }\end{array}$ \\
\hline IntrB13-1 Fw & СTTGCСТСССТATAGGATCACTGC & Pro3-SVMP_Rv & CTGCCATCAGGGGAATAATGAGTCTCACT \\
\hline Met16PI Rv & GATGCGTCCATAATAATAGCAGTG & IntrB13-1 Fw & CTTGCCTCCCTATAGGATCACTGC \\
\hline Prodom $1 \mathrm{Fw}$ & GATGCCAAAAAAAAGGATGAGG & Prodom $1 \mathrm{Fw}$ & GATGCCAAAAAAAAGGATGAGG \\
\hline Prodom $1 \mathrm{Rv}$ & ССТCATCСTTTTTTTTGGCATC & Prodom $1 \mathrm{Rv}$ & ССТСАTССТTTTTTTTGGCATC \\
\hline IntronB7PI Fw & TGGAACAACAGCTGTTGTTATGACG & Intr2 Fw & ACAATGGGAAACTGAGGAACAG \\
\hline IntronB7PI Rv & TGAGAGACATGCTGATGTGGTC & Intr2 Rv & GGGAACTCTGACTTAGAGAAAGTC \\
\hline Met4 PI Fw & GACCCAAGATACATTCAGCTTGTC & Met1PII FW & CAACAGCATTTTCACCCAAGATAC \\
\hline Met4 PI Rv & GACAAGCTGAATGTATCTTGGGTC & Met1PII Rv & GTATCTTGGGTGAAAATGCTGTTG \\
\hline Met8PI Rv & TATCCATGTTGTTATAGCAGTTAAATC & Met $1-2 \mathrm{Fw}$ & CATGGATACATCAAATTGTCAACG \\
\hline Intron B16 Fw & TGTGCTTACCCAACACTGAGCC & Met $1-3 \mathrm{Rv}$ & TGTACATCTGTCAGGTGGACATG \\
\hline Met5 PI Fw & GCACGTGAAATTTTGAACTCA & Met2PII Fw & GCCGTTCACCTTGATAACCTTATAGG \\
\hline Met5PI Rv & GAGTTCAAAATTTCACGTGCTG & Met2PII Rv & CCTATAAGGTTATcAAGGTGAACGGC \\
\hline Met9PI Rv & AGCATTATCATGCGTTATGCG & Met 6 PII Fw & CCACAATCGTCTGTAGCAATTACTGA \\
\hline Met3 PI Fw & GGAAGAGCTTACATGGAGAG & Met 6 PII Rv & TCAGTAATTGCTACAGACGATTGTGG \\
\hline Met3PI Rv & СТСТССATGTAAGСТСТTCC & Met3 PII Fw & GATCATAGCACAGATCATCTTTGG \\
\hline Met2PI Rv & GCTCCCCAGACATAACGCATC & Met3PII Rv & CCAAAGATGATCTGTGCTATGATCc \\
\hline IntrB23PI Fw & CTGACTATGACTCACTTAACAACTGG & Met $4 \mathrm{Fw}$ & ATGATCCAGGTTCTCTTGGTAACTATATG \\
\hline IntrF2PI Fw & GGCCGCGTGAATGCATCTGCTTC & Met $4 \mathrm{Rv}$ & TGAACTGATAGGAACGGTATTGTG \\
\hline Intr2F2PI Fw & GCATCAGTTTGTTCGCACTCAATAAAG & Fw_Ocella NcoI & ATCCATGGTAGACTGTGAATCTGGACC \\
\hline Intr3F2PI Fw & GAGCATAATCTGGAACTAAGATCAAG & IntrDis1 Rv & ATACGGCTAGTATGGAGCAGG \\
\hline Met7PI Fw & GCACAAGATTCCTATCACTTCAG & Dis PII Rv & TCACATCAACACACTGCCTTTTGC \\
\hline Met13PI Rv & TCСТACСТGCAAAAGTTCATTTTC & - & - \\
\hline Intron B10PI Rv & CTGACTCAGGGCACCAATCTC & - & - \\
\hline Met1PI Rv & CTACTGCAGATCGTTCCATCTCTG & - & - \\
\hline
\end{tabular}

\subsection{Sequence Analysis}

Exon-intron boundaries were localized by visual inspection and corroborated using Wise2 [104]. Amino acid and nucleotide sequence similarity searches were done using BLAST [105]. Multiple sequence alignments were performed using ClustalW2 [106]. The occurrence of retrotransposable elements and simple nucleotide sequence repeats (SSRs) were assessed using RepeatMasker (version rm-20110920) [107], a program that screens DNA sequences for interspersed repeats and low complexity DNA sequences included in the Repbase database [108].

\subsection{Sequence Availability}

Pre-pro EOC00006-like PII-SVMP and EOC00028-like PI-SVMP gene sequences have been deposited with the NCBI GeneBank [109] and are accessible under accession codes KX219964 and KX219965, respectively.

Supplementary Materials: The following are available online at www.mdpi.com/2072-6651/8/7/216/s1, Figure S1: Pairwise nucleotide sequence alignments of topologically equivalent paralog introns 1-12 from Pre-pro EOC00006-like PII-SVMP and 1-13 from Pre-pro EOC00028-like PI-SVMP gene sequences.

Acknowledgments: This work has been financed by Grant BFU2013-42833-P from the Ministerio de Economía y Competitividad, Madrid (Spain).

Author Contributions: J.J.C. and L.S. conceived and designed the experiments; L.S. performed the experiments; J.J.C. and L.S. analyzed the data; J.J.C. wrote the paper.

Conflicts of Interest: The authors declare no conflict of interest. 


\section{Appendix A}

Genomic sequence of E. ocellatus EOC00006-like PII-SVMP gene. The locations and identities of the primers used to PCR-amplify genomic sequences (listed in Table 2) are indicated. Protein-coding DNA regions are in upper letters and boldface, and the encoded amino acid sequence is displayed below the DNA sequence. Start of introns are labelled EoPII- $X$, where " $X$ " corresponds to intron number. The beginning and the signal peptide, propeptide, metalloproteinase and the short-disintegrin domains are specified. Numbers at the right correspond to amino acid numbering of the DNA-deduced pre-pro-PII-SVMP relative to the mature SVMP. The $N$-terminal glutamine of the metalloproteinase domain has been assigned residue 1 . The extended $\mathrm{Zn}^{2+}$-binding environment (HEXXHXXGXXH) and the RGD integrin inhibitory motif stand on yellow background. The only two amino acids ( $-70 \mathrm{I} / \mathrm{V}$, and $-111 \mathrm{H} / \mathrm{R}$ ) that distinguish this sequence from that of PII-SVMP EOC00006 (Q14FJ4) are shown in bold and red. The remains of a disintegrin-like domain transformed into intron EoPII-12 are underscored in italics and on cyan background. SINE/Sauria, LINE/L2/CR1 and DNA transposon retroelements are highlighted on a gray background. Simple sequence repeats (SSR, microsatellites) are shown in light green background.

ATG ATC CAA GTT CTC TTG GTA ACT ATA TGC TTA GCA GTT TTT CCA TTT CAA Ggt aag atg

\begin{tabular}{|c|c|c|c|c|c|c|c|c|c|c|c|c|c|c|c|c|c|c|c|}
\hline \multirow[b]{2}{*}{$\mathbf{M}$} & \multirow[b]{2}{*}{ I } & \multicolumn{3}{|c|}{ 5'PS-Disi Fw } & \multirow[b]{2}{*}{ L } & \multirow[b]{2}{*}{$\mathrm{V}$} & \multirow[b]{2}{*}{$\mathrm{T}$} & \multirow[b]{2}{*}{ I } & \multirow[b]{2}{*}{ C } & \multirow[b]{2}{*}{ L } & \multirow[b]{2}{*}{ A } & \multirow[b]{2}{*}{$\mathrm{v}$} & \multirow[b]{2}{*}{ F } & \multirow[b]{2}{*}{ P } & \multirow[b]{2}{*}{ F } & \multirow[b]{2}{*}{ Q } & \multicolumn{3}{|c|}{$1-$ EoPII-1 $\rightarrow$} \\
\hline & & Q & $\mathrm{v}$ & L & & & & & & & & & & & & & & & \\
\hline & & & & & & & & & & & & & & & & & & & \\
\hline tc & tct & $\mathrm{ta}$ & gtt & $\mathrm{CCC}$ & $\operatorname{tgg}$ & ttc & aga & atc & tta & $\operatorname{ctg}$ & cta & aga & cta & $\operatorname{ttg}$ & $\mathrm{C}$ & $\mathrm{cca}$ & $\mathrm{aca}$ & gat & \\
\hline tat & gtt & gtt & ggc & ttt & ggt & tt & att & ttt & gac & aat & taa & cta & aag & $=t$ & $t$ & tca & tt & cag & ttt \\
\hline $\mathrm{Cca}$ & ctg & att & aag & caa & aag & aag & gtt & ctc & aag & gat & $\mathrm{CgC}$ & att & $\operatorname{tgt}$ & tct & a & gtt & aaa & taa & atg \\
\hline gtt & tat & $\operatorname{tgg}$ & aat & $\operatorname{ttg}$ & ctt & aaa & tet & $\operatorname{tg} t$ & aaa & $g C c$ & $\mathrm{agg}$ & ga & aaa & $a c$ & taa & gga & aga & aaa & tcc \\
\hline taa & gat & ttt & cag & aaa & aaa & aaa & aaa & $\mathrm{acc}$ & ctc & att & cat & ttt & aga & 999 & aac & gtt & ttc & caa & $\operatorname{tga}$ \\
\hline $\operatorname{tgt}$ & gag & tta & aaa & aaa & aag & aat & taa & aac & act & gaa & gag & gac & aga & gag & aaa & $\operatorname{gtg}$ & ttt & gtt & $\mathrm{gca}$ \\
\hline aga & aaa & ttt & cag & aaa & $\mathrm{gca}$ & aaa & gaa & aaa & aat & gct & att & gtt & tet & aat & ttt & ctt & tca & ata & ggt \\
\hline cag & tta & gac & atc & $\operatorname{ctg}$ & aaa & tta & aac & atg & cat & aat & att & tag & $\mathrm{CCC}$ & $g g g$ & $\mathrm{aca}$ & cga & $\operatorname{tgg}$ & ctc & agt \\
\hline aga & tta & gga & $\operatorname{tg} g$ & $\operatorname{tg} a$ & gct & tat & $\operatorname{tg} a$ & $\mathrm{Cca}$ & gca & att & $\operatorname{tg} g$ & $\operatorname{cgg}$ & tca & aat & $\operatorname{acc}$ & tag & $\operatorname{tg} c$ & $\operatorname{ctg}$ & $=t a$ \\
\hline get & att & $\operatorname{tg} t$ & tag & gta & taa & caa & agt & gag & $\operatorname{tgc}$ & ctg & tta & ctc & atc & $\mathrm{cca}$ & gct & tct & gcc & aac & caa \\
\hline & & & & & & & & & & & & & & & & & & & \\
\hline
\end{tabular}

gca gtt caa aag cat gta aaa atc caa gta gaa aaa tat ggg cca cta cag tgg gaa ggt cac agc att cta tgt get ttt ggt gtc tag tca tgc tag cca ttt gac cat aga gat gtc ctt gga caa aca ctg get ctt tga ctt tga aat aga aAT GAC ACT GAC CTC TAG AGT TGG Intr4 $\mathrm{Ew}$

gaa atg agt agt atg cat atg tgg ggg act tta ctt tta cct aat att tag cca aat aaa agc tag tgc ttt tga tgt tac aga gac tct tca gac tgt act taa tat aat atg aca cta get ttt ctc aat gca aga ttc cgt att atc tta aag tat ttc ttt cet cet tcc ctt tta ctc ttc aaa aaa tag agc agt ccc ttc tga aat tct tcc caa gtt ctc ctt ctg tga get aag ata cat tt aca tgc cag tta gcc aat cta tgg cta ttc ttc ctg tat ccc aag tca ttc cca cat atc att aca aac aag aat act caa tag att cag aag ttg cca aac aaa ttt aag ggt gag agg ctc act gca gta gcc cto ttt gtt tot ttg ttt gtt tgt tta aat agc aaa gaa aga tga gaa tgc cct tat cag gag taa ttt atg ggg aac aat gta gga aag aag aaa ggg aga tgg aag aga aat tgc tgc tta atc tgt get cta agc aag aga gga ata gt $\mathbf{A}$ AGC TTG CTT GCT AGT AGG TGG aag tga aCC cCt CCC atg ttt ctg gga gat cag gta caa IntrB9_4-2 Fw

ata ggt agg tac atc aat agg tat gag gaa gat gca ttc ttc tgt ctg gga ctg ccc atc aat ctg aaa gga tgc att tcc agg gta aaa tgc tgg tat ctc tgt ttc tcc ctg

tct ctc tgc ctc tct gtc tet ctc tet ctt tet ctc tct ctc tct ctc tca cac aca cac aca gag act ata cat tta cag tat aag tta aaa aac aaa tat CAG GTG ACC ATA CAA TGT Intr4 Rv

CCA tat ggg gaa caa ctc taa tet ttt cca cta ttt ttc taa ttc tga aat cat tga att $\overline{\text { atg }}$ aaa atg tta tta ggt get tca gtg ata act atg ata cta tgg act ctc aat agc ttc ttt tga aag tga aag aaa aga aag tca gca aaa gta tca gga tac cag tga agt agc att tca gct gac agg cat tcc aga taa gat gca ttt gca tag aaa tct aag ttt gcc tag att tta gac taa tgg tta tgc taa acc ata acg cag tat ggt tta ctg aat tgt ctg aat cag att att gtg att ctc atc tta tca ctc agt gca caa act tgc aca ttg gtt gaa atc tat tta ttt ttc taa ttt ttc aac ctc tga aat gtt tcc ttt gca cct gca caa aga aat gtc tcc tgt tag act tcc atc ttt cag aca tca ttc agc aaa tta cat tcc cag aga aaa tct gga ttt ctg tgc tgt gtc cca ctg gga aaa gat tcc att gtt cac tga taa ctg aat aac aaa ttg tgt tgc aga tgt gaa att gat aga ata aca tcc caa aga aaa cca aat ctc ctt ttc ttt ttc ctt aca aag GG AGC TCT AAA ACC CTG AAA TCT GGG AAT GTT AAT GAT TAT $\begin{array}{lllllllllllllll}G & S & S & K & T & \text { L } & \text { K } & \text { S } & G & \text { N } & \text { V } & \text { N } & \text { D } & \text { Y }\end{array}$

GAA GTA GTG AAT CCA CAA AAA ATC ACT GGG TTG CCT GTA GGA GCT TTT AAG CAG CCA GAG Prodom 3 Fw/Prodom 3 Rv

Figure A1. Cont. 
tat tcc tte cet get att taa tgg tta ttt gga ttt tgc att gca atc tat get cet gtt tga aat att tat tta ttt att tat tta ttt att tat tta ttt att tat tta ttt att tag caa att tac act gcc tcc cag ttt aca caa aat tga gga taa tcc tgg caa ttt ata aac aga att tcc taa tat taa tag gtt aat ata act aat caa gtg ttg gga gaa agc cag cca tac agt gaa gag gaa tca agc agt gaa ata acc att agt cat ttc ata ctg caa cag atc cag caa aga cat cct ctc cct cct cca gga aat CCC att aaa gtt tat ctg atc tgc aga gaa tgg aag cag tct agg gag agt cct gta tta cag gca aaa aat aat aaa ctg ctt aat tta aac ttt gta tat cta cat gcg tca cat aga aat ata aaa gat ata ttt cta gat atg ata gta tag tgg tta cag ctc aag gtt agt tct ata tac cag att cat gta tag cgt ggg atg act ttt gag tca atc tgt ctg tcc cag atc aac ctc att cag caa tat gtt cag tta gag aat gag atc ttg aac cta tgc agg aaa aat aaa taa aga tct tat cat tca aag cac cag gtg aaa tac tct aat aat aat aat cta ata aat aat atg act atc aca aat tat tca tta gat tta aat taa tac aga tgt aga gta tta aga aat gac aca ttt tat ttg cct aaa ttt tga gaa gta aaa cat aaa ctc ttt gtt ttt cag TAT GAA GAC GCT GTG CAA TAT GAA

\section{Prodom $2 \mathrm{Fw}$ /Prodom $2 \mathrm{Rv}$} TTT GAA GTG AAT GGA GAG CCA GTG ATC CTT CAT CTG GAA AAA AAT AA $\begin{array}{cccccc}E & \text { A tat } & \text { gtt } & \text { aac }\end{array}$

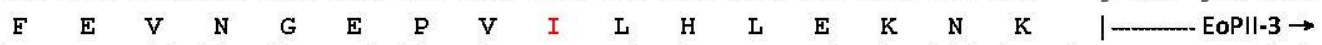
tca gaa att ttc tta act tta cta aac aat gtg gaa aat gta ttt tcc tgg aca caa tct gac aga aat aat aat $\operatorname{tgc}$ att cct ttg ttt get ctt aaa tta aat gtt act ata tag gaa aag tgg ata tgt taa tta tgt ctt acc ttg gaa ctg aaa ttt ttt tac tgc tgt tct cct atg gga cac toc tga act ata aca att tta gac tca gtt gaa cca ttc aag gct cat aaa cgt cta tca ctt gaa tta act agg tta gtt atg aaa tat tgc aga gat ttc aaa atg ttt ctt tat get get tca tag aca ctc cat caa tet gaa taa cat ttt ctg tga gac ctg tag agc tgc tca atg cac atc aaa att tat atg tta aat cat gaa agg cag taa atg tca tct cct att aag gcc cga aag ggg aca gga cat aaa taa gcc cag att tca caa gcc ttt ttt aaa aga atg cet tgt gag tet tct cag aat tgt tcc att tga act gta aat caa gat att aat aaa aat att ata caa ata tga acc aca aaa atg gaa tga aat tat ttt tca gca caa aag aca aac ata gtt gga agg gac ctt gga ggc ctt cta gtc cac ccc ctg ctg aag cag I- LINE/L2/CR1 -

gag act ata tca ttc cat ggg tgt cca att gtt cct tga aaa cct cca gtg atg gga tac cca cag ctt ctg aaa get agc cat tcc acc gat taa ttg ttc tca ttc tca gga aat ttc ttt tag ttc tag gtt gaa ttt ttC ACA GGT AAA TAA GCC ACA AAC ACC att aaa aga aag Intr3 Fw

tgt gcc att ttc att acc cta aaa act gta aat gtc ata aca cag ttt aaa tta gtc tgg cag tca cta aac tgc aaa cac gta cct cag cct ggt tca gtt taa tac tat agt taa GAC TGA GAT ACA GAG TTG GTG Cat tgg gaa aga ctg CCt gCC ttt gat ttt agc atg tat $\overline{c t t}$ Intr3 Rv

ggt ttt gat cat tet gca tgt acc ttt aaa agt ata ttg tct tta tta tta aac ata tgc tac aag aaa ttg cac tat atg tta gta tct gtg tgt cac ttc ctc cta ttc cac cgg aga tat ttc atg cta aat tct aat gtg tgc ttt aga cgt tct agt gtc ccc ttt tgt ttt gtt taa ata tga agg cta tac aga aat tca agg gta gca tat gga tgg tet tct ttt gtc ata gaa gca aag cag ggc tac agg ggg aag acc agc aag gat ttc tag att ggc aaa aat gga aga cga tga caa gtt tat ttt tct gac ttc agg att gga atg tga ctc cat ata ctg gtt taa agc ttc aaa ata tat ctt tct gta tga atg ttg aag gca cac att tcc ttc agg ttg ttt cat ttt acc ctt tag taa ata gaa cta tac cgt ttt cct tta gag aca aag cag ttt cat cag att gtt gca tag aga aga aga ata aaa gga aga cgc aat aca aac tgt cct ctc ttt gtc cat cac aat cet ctg agc att gat gaa gag aat tgc aca get tca tet ggt gaa tct agt atg cac tgc ctt cct ctt aaa aag cca tgg gat aga aat acc tgc tcc att ctt ttg ata cca aag aag ata ctt aaa ttg cca tta tac ggt tgt ttt aaa ctt ctt ata aaa tta tct ctg tgc tac ata att cct gat ata gat atc ctt tct ttg cat tct ttc cag A GGA CTT TTT TCA GAA GAT TAC AGT GAG ACT CAT TAT TCC CCT GAT GGC AGC GAA ATT ACA Pro2-SVMP_Fw/Pro3-SVMP_Rv

$\begin{array}{lllllllllllllllllllll}G & \text { L } & \text { F } & \text { S } & \text { E } & \text { D } & \text { Y } & \text { S } & \text { E } & \text { T } & \text { H } & \text { Y } & \text { S } & \text { P } & \text { D } & \text { G } & \text { S } & \text { E } & \text { I } & \text { T }\end{array}$

ACA AAC CCT CCT GTT GAG gta ggg tet cac ttt tat gag cet tet ttt tag gaa gta aac

\section{$\begin{array}{lllllllll}T & N & P & P & V & E & - & - & -\end{array}$}

tga aac aaa tgt $\operatorname{tg} \operatorname{tgc}$ aca ata tta caa ata tac aag aat gag acc agg cta ctc aaa caa agt gta tat atg tat gaa gta ttt tat att gat atg tac gta caa gga tgc ctg gat tgt taa acc ctg gtt aaa agc caa cat att tgg gag gtg agt ttc aca aat aga ttt att atg aga aca tca ggt ttg taa gat tat att ttc att ttt aaa cca gac tac agg gat aaa tgc aaa gtc ttt tat aaa tga taa caa ttc act ttg ctc cta tac aga aat cca ttt aat atc ttt cat aat aaa atg gtg cca aaa atg get cta tca gat gta aaa caa tta gag cac taa cat get tca tot tgg ctc cat get CCC aaa ttg att taa aag tgc att etg tgt cta ttt ctg gtt tat tat ctt caa gag tca cac aaa tta ctc ttt tgt gtg atc agt ggg agt cCC ctg cag tgt gac ttg att tat gga gac ttg cat tta tcc tat gtt cat ttt gca aca atc aat att aag aag gtc ttc ggt tct cet gaa tca aaa ttt tct gga aaa att ctg tct aaa tat ttc att gat gtt ctg gaa tac att gga act gta ctt ctc ctc atc aaa tca caa tac aaa caa get tta aCC agt gta gtc ctC TTG CCT CCC TAC AG GAT CAC TGC TAT TAT

IntrB13-1 FW

CAT GGA CAC GTC CAG AAT GAT GCT GAC TCA ACT GCA AGC ATC AGC ACA TGC AAT GGT TTG

Figure A1. Cont. 
$\begin{array}{llllllllllllllllllll}\text { H } & G & \text { H } & \text { V } & \text { Q } & \text { N } & \text { D } & \text { A } & \text { D } & \text { S } & \text { T } & \text { A } & \text { S } & \text { I } & \text { S } & \text { T } & \text { C } & \text { N } & \text { G } & \text { L }\end{array}$

AA gta aga tag tet cta atc ttt tat ttg ttt att aat aat aat ata gtg ctc ttg gag K $1-$ EoPII-5 $\rightarrow$

ttc taa ttg tta aaa tga agg aca tcc tca gtt ttt cat gga aat tag ttg ggt gtg atc cag gat ttc ggc aga att aag aca tac ttt ggt tga aaa cca aga aga get get gcc agc cag gag aaa aac tat gga get aaa tca cat aag tct aaa gga get tcc aag ccc cgg tct cct ttc cca ggg tga ggt gat att aca ggt aga gaa gat tag tag gtt tca aat tgg aga cct tgc tag aaa gtg tac agg aag agg caa gaa gtt tca gtt cta ccc aga aac act ttc ttg agt cac tct gca cac ttt ctt cag cca act aga tat gtt aac tac ata aag atc cca gaa ttc aga agg tcc cta tca ata gta aga atg aac atc acc tca aca tet ttt act gaa aaa aga cac tga aac tca cct ttg aac aga gac tgt gtc cat gga gtg gag gaa taa atg aaa agc tgg aac aga gca gaa taa caa cag aaa aat aaa gga aaa aca gaa tga cag aat

aat agc att gga agg gac ttt gag gtc ttc tag tcc aac ttc ctg ctc aag tag gag acc LINE/L2/CR1 $\rightarrow$

tat atc atc cta gac aaa tag ctg tca atc ttc tct taa aaa gca gta gtg atg gaa cac cca caa tgt ctg aat agg tta att gtt cca ttt gtg aga aaa tta ctc ctt agt tct aac tta ttt ctc tct ttg gtt act ttc cac gca ttg ctt ctt ctc ctg cca tca ggt gaa gaa tag gtt gtc cca cat ttt tta tga cag cct ctt aaa tac tta aag att atc aag tca tct cta ccc ctt ctt gtc act agc atg agt ata ctc att gtc tgc agc cat tct aac cct cca gtt agt atg cat tct tat tcc tet cat tgt tac tcc ctg ttg ttc tgc att gac ttc tct atg aga tgc ttg cca aga atc tat ttc att att tat aaa tat cct gtc atc tga ctc tat cta aat tgc tat caa act aat ctg att tta ttt cct tga cca cag aca aat att gtt cta tac ttg ttt aaa gta aat tgc agt att acc tat aac tct ttt tag ata ttt tag cag tta tat ttt tcc ttt tt atc cta ctt agt tgt gat tct tga get tta tca gta ata tat atg ata aat ata aag tat ttt acc ctt atg aaa taa agt ttt aca caa agc aga atg tta caa ttg gct tta gtg ttg tat tta tgt agc tag aaa ctt att ttt tta aca tcc tgg aaa tat aca ata ttg ggt tcc atg cca aaa tat ttc caa aca aaa ctg tac acc tat ttt gtg gct gca ctg agt ttg tga aat ctc tca tat ctt tct gat cat aac tgc atc tat gaa aag tat gag aaa gtg att tga gtg ctg agg aaa gaa tat aaa ata ttc act cat tgt taa gaa gga att caa aaa cat gag gtt agt tga aaa tgg gtc tca gag ccg agt ttc att acc caa cta ggt aac atc atc agt gca gtt ttt ctc tga act aac aat att ctc ttc ttt tgc ttc tcc atc tet gat cat cet tet cac att gtt tta cag A GGA TTT TTT ACG CTT CGT GGG GAG $\begin{array}{lllllllllllll}G & F & F & T & L & R & G & E\end{array}$ ACG TAC TTA ATT GAA CCC TTG AAG GTT CCC GAC AGT GAA TCC CAT GCA GTC TAC AAA TAT $\begin{array}{lllllllllllllllllllll}T & Y & \text { L } & \text { I } & \text { E } & \text { P } & \text { L } & K & \text { V } & \text { P } & \text { D } & \text { S } & \text { E } & \text { S } & \text { H } & \text { A } & \text { V } & Y & K & Y\end{array}$ GAA GAT GCC AAA AAA AAG GAT GAG GCC CCC AAA ATG TGT GGG GTA ACC CTG ACT AAT TGG Prodom $1 \mathrm{Rv} /$ Prodom $1 \mathrm{Fw}$

$\begin{array}{lllllllllllllllllllll}\mathbf{E} & \mathrm{D} & \mathrm{A} & \mathrm{K} & \mathrm{K} & \mathrm{K} & \mathrm{D} & \mathrm{E} & \mathrm{A} & \mathrm{P} & \mathrm{K} & \mathrm{M} & \mathrm{C} & \mathrm{G} & \mathrm{V} & \mathrm{T} & \mathrm{L} & \mathrm{T} & \mathrm{N} & \mathrm{W}\end{array}$ GAA TCA GAT GAG CCC ATC AAA AAG GCT TCT CAT TTA GTT GCT ACT TCT GAA gta agt ctc

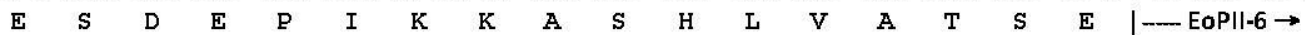

ata gta aac ata gtt taa gat cac ata ctc att tgc ttg ttt aga aaa tat aaa gta aga gag aaa ttc ctt tgg gga gag gtg ata gat aga att taa aat gga gaa gcc ccc att tct ata ttt tta ttg taa agg taa agg taa agg ttc ccc cac aca tat gtg cta gtc gtt tct SINE/Sauria $\rightarrow$

ggt ccg gtg ctc atc tcc gtt tca aag ccg aag ggc cag tgc ttg tct gag gac ata tcc gtg gtc atg tgg ctg gca tga cta gac acc aaa ggc gta cgg aac gct ttt tcc ttc cca ccg tag tgg tcc cta ttt ttc tac ttg cat ttt tac atg ctt ttg aat tgc tag ggt ggc aga agc tgg gac gag taa cag geg ctc act ctg tta cac agc act cgg gat ttg aac cgc caa act get gac ctt cag atc aac aag ctc agt gtc cta acc cac tga gcc act gcg tcc ctc att ttt att gta gCC aag gta taa aag aaA CAA TGG GAA ACT GAG GAA CAG aaa ata Intr2 Fw

aat ctt cca gtt gtt tga tca aac aaa ctt agt ttg gag att tga atc aaa aat gga tt aaa tga gtt tct aaa tca tct cca ctt tct aag tca att ttg aaa agt aat taa att atc aat ttg gat gcc tct ttt atg cat gca gaa aga aat tga gat ggg gga gaa agt ggt ttg aaa tat tta atg gtt tta aga tgt ctg ata agg cca tta cat aat tgt gac tcc att ttc cag ttt gat ttg aat cat caa gtt gga ttg atg caa tga tgc aat aac tgg atg gaa agt ggc aat gtg aac cta gtc aca att ggc cet tat get ctc aat gtt ttc ctc ctt tac tgg agg cac aaa aat tag aaa aca aaa tat tgc atc caa agt gac agt tcc tta cat ctt ttt ggt ggc aaa agt tga aac tgg ctc aaa aat ctc aac tgt ttt tat tag aat gtt aaa att gac atg gaa caa cag ctg ttg tta tga cgg aat acc aaa aca caa gtg aag acg cca aat gaa gtc ggg ttt gtc ttt tgg ctt ctt tca ttc tgg caa ttc aag att ctt tat cct cag caa ttt gtg gtt ata cgt tac att taa ctg gtc ctt aat tgg ttc ttt atc ttg aat gtt ctt gtc tca aag tca gtc atg atc act tta ttc gga ctc gat ctt gtg cag cag aat taa gaa agt ggc tgt gag agg aga agg aga gaa att gca ttc tag aat tgc aac ttg gct tgg tag ttc tgt aca ctc tca tag gga aag aag caa tta atg cac act cac aca ttc agt ctt agc ata agt gaa ctt cct gag caa cct ggg ttc tct agt gga gga atc tcc ctt tgg aat gca gga gag agg ggc tct ttc cta gag aaa taa gag gca gat cag aaa tga atc ctt gga ttg cag aaa gat gcc cgg aag gga tcc tgc cta aag cet tca cag gat gta tct cat ggt tac acg gag tgt get tta gtc tgg caa gat tcc tgt cta cag gag aag agc aat aaa gaa act act ctg agt aat taa cca cag ttt ctt gtt cet tgt gct caa gaa gtc tga aac aat att tca gta atc att tta aaa ata cat cac tga aaa ggc ata att ctt tgg cca tta aga gtg agt tca gtg ggt caa aat gtc tat ttg ctt ctc ctt acc ttg tct ttg atg cat agt gtg ata tga ttc cag gcc agg tct aat tgc atg acc aca tca gca tgt ctc tca cgt gat

Figure A1. Cont. 
tgg ttg gca tcc ttt gtt tga gaa agg gaa gga aag ttg aga aag tca ttg aag cat cat ttt gac agg gtg aaa aac acg tca aag aga aca gtt tcc tca tat gcc tat taa att ctt ttc aga gtt agg tat tca tat ata cca tta tct tga caa tcc att gaa taa cgt act ttt ttc ttc aaa act tta tca tGA CTT TCT CTA AGT CAG AGT TCC Caa acc ttt cCa get ttg Intr2 Rv

ggg ata ggt gag gga gag ggg atg gtt cca cgt gaa cag tgg ggt cag gtg tgt acc cag ctc tat ttg tgt gag cag tgg gca cac ata ccc act cgt gta aac aga gca cac cca cct atg ctt gtt cac tgg tca tac aag tag aga tgc agc tgc tca cct gcc att tcc atg gcc cag ttc tga agg gct gca ggc cca ggg cta aaa ttt taa caa gct gtc tcc ctg taa ata tct tct tga aag aac tga tat ttc tgg aag ttg acc aga gag taa aac aag cat ttt tct gat tat ctg agg ttg acc aac gtt ctt gtt ggc tgc tgg gag taa cat gtt taa aca gcc att tac tet tCc gtt tag CAA CAG CAT TTT CAC CCA AGA TAC GTT CAG CTT GTC ATA GTT

\section{Met1PII Fw/Met1PII Rv}

\section{$\begin{array}{llllllllllllll}\text { Q } & \text { Q } & \text { H } & \text { F } & \text { H } & \text { P } & \text { R } & \text { Y } & \text { V } & \text { Q } & \text { L } & \text { V } & \text { I } & \text { V }\end{array}$}

GCA GAC CAC TCA ATG gta agt atc ttg gat atc ttt cta ttt act ttt tgc att gag cgc A $\quad$ D $\quad H \quad$ S $\quad$ M

agc att tcg ttt tgg cet ttt tta atg tgg gca ttt ttc aag aga tta tcc taa ctg aac ttt ctc tta aaa tgc get tta tca taa act ttg ata ttt ttt gtt att gga ccc aac caa aat tta caa agc taa aag tca ttt gta aat ata ttt taa ttt gca cac ttg tta ttc ctg gat acc att taa gtt tat tt tat $\mathrm{ccc}$ aca ctg gtt aaa aag ttc tgt agg ttt ctt tga aat gtt $\mathrm{CCC}$ acg ctc ttt tt $t$ tg tcc aag ttg gca aca tac aaa gaa aaa agt gaa gaa tgc tta tct cac aca tct ctg aaa gag gaa ata ttt tct cta acc agg aaa aag gcc cat gta tgt tgc tga aaa gtt aaa aat ctt aat ata tta atg gca caa atg tag att aaa aaa aat agc tca aaa gaa ttc ttt gga ctg ctt gga taa aaa tta tta cat caa gaa att caa aga tcc ttt cac taa tat at ctt tet ctc ctc cet tct ttc cet tct tat aat tat caa ctt gtc tta act tet tet ttt tgt ag GTC ACG AAA AAC AAC AAT GAT TTA ACT GCT TTA $\begin{array}{llllllllllllll}\mathrm{V} & \mathrm{T} & \mathrm{K} & \mathrm{N} & \mathrm{N} & \mathrm{N} & \mathrm{D} & \mathrm{L} & \mathrm{T} & \mathrm{A} & \mathrm{L}\end{array}$

ACA ACA TGG ATA CAT CAA ATT GTC AAC GAT ATG ATT GTG gta aga aca aat get tgt tca Met 1-2 Fw

$\begin{array}{llllllllllllll}\text { T } & \text { T } & \text { W } & \text { I } & \text { H } & \text { Q } & \text { I } & \text { V } & \text { N } & \text { D } & \text { M } & \text { I } & \text { V }\end{array}$

1-- EoPI I-8 $\rightarrow$

ttt taa act tca ctt agg $\mathrm{ccc}$ agc cga gat ttt gat tgt gtt aag ata aca aac ata atc agg taa ata aag tag atg gat ttc taa atg caa acc tct gct ccg cac gct gca ttg gct

LINE/L2/CR1 $\rightarrow$

ccc agt tgc tct ccg ggt gag att cag tgc tgg taa tga cct ata aag ccc tac atg gct tgg gtc cag aat atc tga ggg aac acc tgc agc caa gtt ctc atc gtc cgg tac gct ccc aca ggg agg ggc tcc tta gag tac cgc cgg caa agg att gcc gge ggg tga ctc cta gag aga ggg cct tct ctg tgg gtg cac ccg ccc ttt gga acc agc tcc ccg tgg agt tga gga ctg ccc ccg acc tgc gtt ttt ttc gga gga acc tga aaa cat ggc tgt tta atc tga ccc agg ctg gtg ttt tta gat ttg ggg ttt taa ctt ggt ttt aat ttt gag gat tgt gtt taa LINE/L2/CR1 $\rightarrow$

tgt att ttt agc tgt ttt tta att ttt gta ata ttg tct ttt aaa ttc ctg tac acc tcc ctg agt cct tcg gga aaa ggg tgg ttt aaa aat aga att aaa taa ata aa ata aat aaa taa atg aat ggt ttc ttt tgt ggc ttt gag tga tct aaa act atg tta ttt ggc cag ctg gtg atc cag cat gtg agg cca tcc cag aaa atg ggt taa ttc agt aac taa att aat aag cct cta atg gaa ata gag tca ttg ggt ttg gag gat gca aat cca aaa gtg ttg caa gaa ttc agt cag aag tat atc tgt agt tgg att ctt tca ggg ctg tgg tta atg gtt ctg agc ttg gag att aaa aaa tga tgg aca gag tca ggc tat tcc aag ctc agt tga tta tga aaa tga tct ctg agt aga aac ctt gag aga aga act caa ttt gac ttg gat tgt ggt act tag gag gag ata cag tca tat tgc att ctc cga tta gcc ttg ttt aca ttt tca cta ttg atg atc agt tag aaa cag gga gag aac agg aag atg gaa gaa tca act ctc ctg tta gtc ctc cta ctc ttc tgt tcc ttt tta cag aaa ata atc acc tgc ttt ttt tat cat gtt att tat tag agt cct tag tac tgt cta agc ttg gtg gtt acc ttg cag aca ttt cat tat ctg act act cta cat gag taa cca aag cag caa gct cat agc acc aag gac ttc aac ctt gag ctt cta ttg atg cet agg tct att ttt ttt tca tct aca aaa aga ggc caa tgc cag ttg ctt ttc aat att gaa atg ttt ctc aag gtt tac ttt gtt tga gtt tct gac tga tgC CAT GTC CAC CTG ACA G ATG TAC AGA ATT CTG AAT ATT CAT ATA ACA CTG GCT AAC GTA GAA ATT Met 1-3 Rv

$\begin{array}{llllllllllllllll}M & Y & R & I & \text { L } & \text { N } & \text { I } & \text { H } & \text { I } & \text { T } & \text { L } & \text { A } & \text { N } & \text { V } & \text { E } & \text { I }\end{array}$ TGG TCC AGT GGA GAT TTG ATT GCT GTG ACA TCA TCA GCA CCT ACT ACT TTG AGG TCA TTT $\begin{array}{lllllllllllllllllllll}\text { W } & S & S & G & D & \text { L } & \text { I } & A & \text { V } & \text { T } & \text { S } & \text { S } & \text { A } & \text { P } & \text { T } & \text { T } & \text { L } & R & S & F\end{array}$ GGA GAA TGG AGA GCG AGA AAT TTG GTG AAT CGC ATA ACG CAT GAT AAT GCT CAA TTA ATC $\begin{array}{lllllllllllllllllllllll}\text { G } & \text { E } & \text { W } & \text { R } & \text { A } & \text { R } & \text { N } & \text { L } & \text { V } & \text { N } & \text { R } & \text { I } & \text { T } & \text { H } & \text { D } & \text { N } & \text { A } & \text { Q } & \text { L } & \text { I }\end{array}$ AC gta tgt ctc att gtg ggg aaa ggg agt gag agt ggc tgg gag tgg agg att atg gaa T $1 \longrightarrow$

agg tta atg ctt gcc tag agc ttc tgt tct atg ctg tat get tta aac cat gca tgt agt aca ttt ctg ggt caa agt caa cca ctt ata tta tag atg aga cct ggc tt gag aaa tct ttg aat gat tgc agg tga aaa atc cat taa ata aat atg tat ttg ggg ttt gca tag tta atg gaa tta aat taa ccc aaa tgg gtt gga ttt gac aac atg cta acc cet ctc ccc aac ctc att cat get aat gcc agc caa aca caa cat ata gtg aaa aat ata tcc tga ata tt tta acg gac ctg ctt aac taa atg gct ttt gag act aga gcc taa aat gaa ttc tag cat tct gaa aac tgg tag taa ctg agg acg ggt caa agg gtt tac aga aat cca tat tta tgt atg acc taa gac tac ata aca tgc ttc tat ctt cta tca att tta tcc ccc tcc cet tct

Figure A1. Cont. 
tet tta tag A GCC GTT CAC CTT GAT AAC CTT ATA GGA TAC GGT TAC TTA GGT ACT ATG Met2PII Rv/ Met2PII Fw

$$
\begin{array}{llllllllllllllll}
A & V & H & \text { L } & \text { D } & \text { N } & \text { L } & \text { I } & \text { G } & \text { Y } & \text { G } & \text { Y } & \text { L } & \text { G } & \text { T } & \text { M }
\end{array}
$$

TGC GAT CCA CAA TCG TCT GTA GCA ATT ACT GAG gtt agt aga aag gat act tta tta tCt $\begin{array}{lllllllllll}C & D & P & \& & S & S & V & A & I & T & E\end{array}$

att tgt act caa gtg aaa cct tac ata cag aca aaa cat ctt ttc aaa taa agt ctc tt ctt att ttt gag cca cot cat ttt cac cca tat tta ttt gca gat ttg aca tct cca ggt cct gcg tca act aat ggc att ttg aca cag tgc att cta gaa caa gct ttt tta atg caa tga gct ata tgt caa gga tga gaa tat att ata atg ttt atg gtt cag tca aac tgt act ctg att ggc aaa tga aca ggt caa agc atg tta caa cac ttc caa ata atg ctt ctg aac aat agt ctt agc aat $\operatorname{ccc}$ aaa gac aaa cat gaa ttc att cca aga aat tta gtg tct aga ttg cat atg att gaa ttc tag tac att gag aaa aca aaa aaa tac taa atc tac tca aaa aga aaa aaa acc ctc tag ata tta gtt aag gtg atg cta tgc att tat tga gaa aga gta aac tta gct ttt tgt tca cat aga aag aat gga gag aca tgg taa taa aca aaa gtt ata caa caa aac tca taa agt ttt gtt tct taa taa gca gag tta gga tcc tgg tag tag tag gta ctc ata agc cta ctt gct caa gaa ggt tat ttt att cag aaa gag caa ctc att cta agt ctg ttt agg atg gct acc ttc aat att ctg aaa atg caa gat tgt agc aaa gga cac tga gta gtt ttt ttc gac tga agt ttc ctg taa gtc agg get gtc aaa ctc aat ttc att DNA transposon $\rightarrow$

gag ggc cac atc agc att gcg gtt gcc ctc aag ggg gtg gtt ggg tgt ggc cag ggt ggg cac agc cca cag gca tgg ctg gaa tgt ata tgg cta agt ttt agt aac tga ata agt gca gac agc aaa tgg atg cat aca ttt tga tct tat tct gtg ctg tag ctt ctg get gta aag tet cet tet gga tgt att tgt gta tgt tet gga gtc ttg gtg ggc aca gat act ttc aga gga get aga gga atc ctg aga tgg tat cct caa cct aaa att ggt cac ttg gtc acc agt ttt agc cac tta gtg gta ata ata att gga ttc act ttc agt ttc ttg gca gag taa caa taa aaa aag tat tet tat ttc ttc ag GAT CAT AGC ACA GAT CAT CTT TGG GTT GCA GCT Met3PII Rv/ Met3 PII Fw

$\begin{array}{llllllllllllllllll}D & H & S & T & D & H & \text { L } & \text { W } & \text { V } & \text { A } & \text { A } & & \end{array}$ ACA ATG GCC CAT GAg ATG GGT CAT AAT CTG GGT ATG AAT CAT GAT GGA AAT CAG TGT AAT

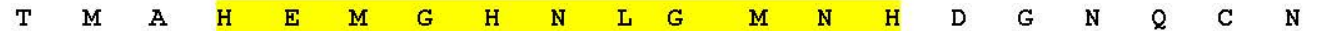
TGT GGT GCT GCC GGA TGC ATT ATG TCT GCG ATC ATA TC gta agt att gag gaa tat get $\begin{array}{lllllllllllllll}C & G & A & A & G & C & I & M & S & A & I & I & S & \end{array}-$ EoPHI-11 $\rightarrow$ taa tgg ctt tcc aat caa gtt att ttt aaa tgg ttg caa aaa tga ata aag tat tct ctt atc cat tct gtt agc ttt aga aga aaa caa atc att aca ttt ctt cat tag caa ttc ctt ttc ctt ata tgt ttt tgc aat gaa att ctg ctc cta gtc caa agt tgg agg atg tca tga tet tet ttc ata tet aca $g$ A CAA TAC CGT TCC TAT CAG TTC AGT GAT TGT AGT ATG Met4 Rv/Met4 Fw

$$
\begin{array}{llllllllllll}
Q & Y & R & S & Y & Q & F & S & D & C & S & M
\end{array}
$$

AAT GAA TAT CGC AAC TAT ATT ACT ACT CAT AAC CCA CCA TGC ATT CTC AAT CAA GCC CTG $\begin{array}{llllllllllllllllllll}\mathbf{N} & \mathrm{E} & \mathrm{Y} & \mathrm{R} & \mathrm{N} & \mathrm{Y} & \mathrm{I} & \mathrm{T} & \mathrm{T} & \mathrm{H} & \mathrm{N} & \mathrm{P} & \mathrm{P} & \mathrm{C} & \mathrm{I} & \mathrm{L} & \mathrm{N} & \mathrm{Q} & \mathrm{A} & \mathrm{L}\end{array}$ AGA ACA GAT ACT GTT TCA ACT CCA GTT TCT GAA AAT GAA CTT TTG CAG gta aga gaa gaa $\begin{array}{lllllllllllllllllllll}\mathrm{R} & \mathrm{T} & \mathrm{D} & \mathrm{T} & \mathrm{V} & \mathrm{S} & \mathrm{T} & \mathrm{P} & \mathrm{V} & \mathrm{S} & \mathrm{E} & \mathrm{N} & \mathrm{E} & \mathrm{L} & \mathrm{L} & \boldsymbol{Q} & \mathrm{V} & \mathrm{R} & \mathrm{E} & E\end{array}$ I- Spacer -||$-$ EoPII-12 $\rightarrow$

tgt gac tgt ggt tct cet gca tta agt ctt ttt ttt taa tca aca aaa gta att tga aga $\begin{array}{lllllllllllll}C & D & C & G & F & P & A & L & S & L & F & F & \text { Stop }\end{array}$

ata ttc tca gaa atg aga atc ctt gaa aaa tca tct agc ttt cta agt ggt ttg agc cat cca aga ggt tgg ctt gtg aat gge tga ggt ttg tgc ctt tca tgt aca tgc atg tat gaa gtg gtt tct tgg gtt gta gag gaa tgg aga act ggt atc tca cta cta ttt tgg gaa gat ggt gaa ttt tta aaa acg ggt gat tga cca ctc caa gaa aat ctt tcc ctc ctg aaa ccc cct att ttg ttg ata tag cca cat tat cet gta cca cga ttt tet cga act gct cCc tcc cat atc tga tta tct tta atc tat get ctg atc cta ata ata ttt tta taa gaa cag taa tat agt gtt ttt atg ttg tta aat aca cct gtg atg gtc tot gag aat gtc ctt aag aga caa aag aag gac gaa aca tcc agt taa tgg tcg tat aag aag gag att aac ctg cag aag caa tgg caa aaa tct caa gat gga cac ttc cca ccc att ctc ttg gtc cgt aaa gat gag gtg gta caa ata gat ttt cag tat tga aag att ctg cta ctg taa cct tac aat cat gtc gca tta ata ctc aag get get get tet tet cta gac taa ctc aaa ggc tgg cat gat gag tag aaa atc cat cat gaa taa gaa agg aat ggg get gta ggt tat gtg ggc ttc aca att agg aga tga gga tat ttt tgt ttt att ctt ttc acg tag gaa ata tca gat aag gct ctt tcg cag aga aat gcc ttt agc tgt ttt caa taa caa aca att tgt gca tct cet agc atg aac tca taa gag goa aca tat cgc aga aat gtt cet ctt caa aat aga cca att aaa aaa gaa aat tct atg cCa tca ttc gat atg ttt tgg ttt tca g AAT TCT GTA AAT CCA TGC

$$
\begin{array}{lllllllllll} 
& N & V & N & P & C
\end{array}
$$

TAT GAT CCT GTA ACA TGT CAA CCA AAA GAA AAG GAA GAC TGT GAA TCT GGA CCA TGT TGT

$\begin{array}{llllllllllllllllllllll} & Y & D & P & V & T & C & \& & P & K & E & K & E & D & C & E & S & G & P & C & C\end{array}$

GAT AAC TGC AAA gta aga ctt att tat ttt taa cac caa gag aaa ttt tac cct gct cca $D \quad \mathrm{~N} \quad \mathrm{C} \quad \mathrm{K} \quad 1-$ EoPII-13 $\rightarrow$

tac tag ccg tat aga aat ata ata ttt ctt ggc tgt tta cta tga taa aaa cat ttc agc IntrDis1 Rv

tct att tcc tat ecc ttc ttc cag ttt att tga ccc tta tga aca taa gca aag gga aga taa ttt aac aaa att tet ccc tta ttt caa ttt caa atg cac tct ttc agc atg cta aat

Figure A1. Cont. 


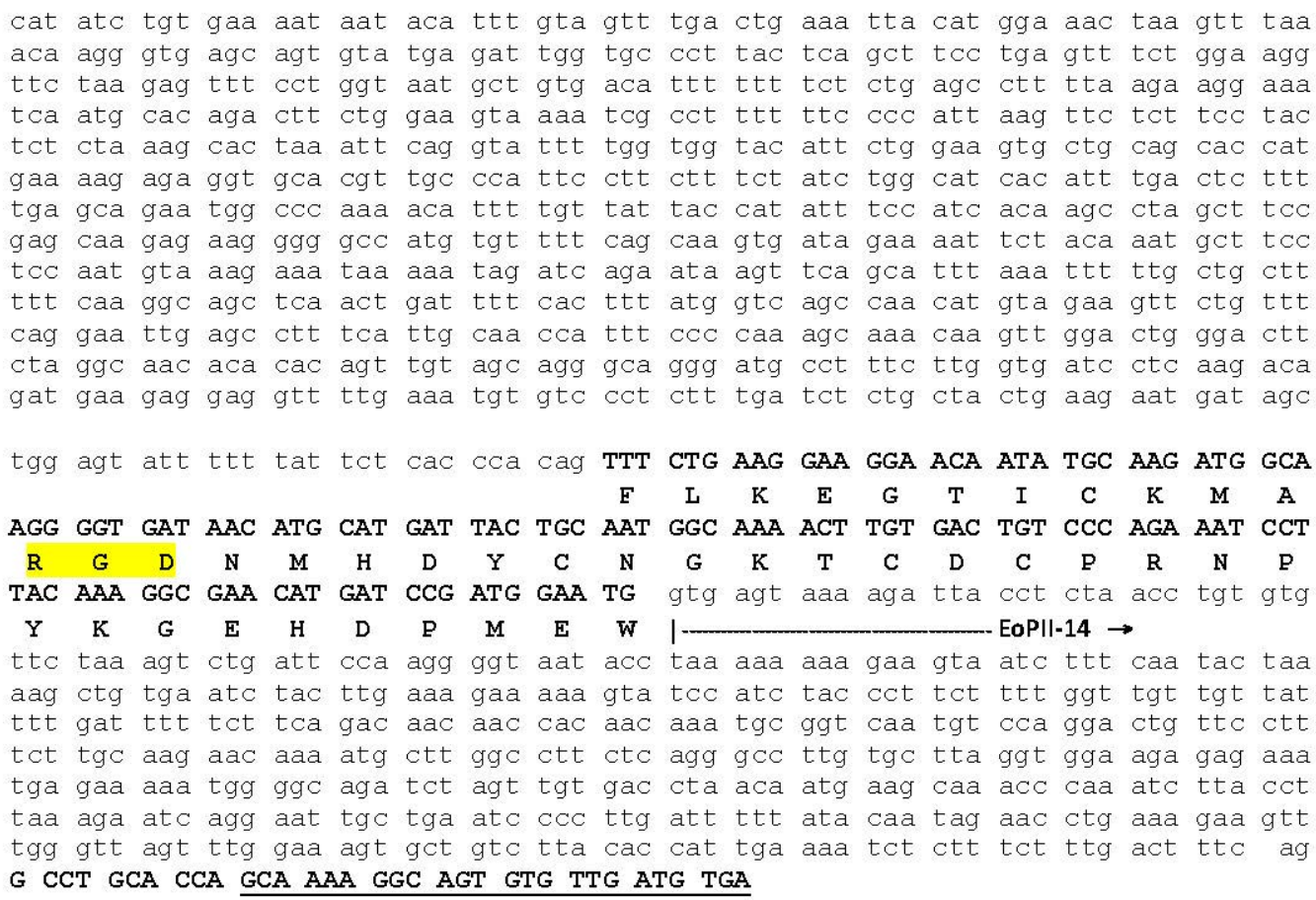

Figure A1. Genomic organization of E. ocellatus EOC00006-like PII-SVMP gene.

Genomic sequence of E. ocellatus EOC00028-like PI-SVMP gene. The locations and identities of the primers used to PCR-amplify genomic sequences (listed in Table 2) are indicated. Protein-coding DNA regions are in upper letters and boldface, and the encoded amino acid sequence is displayed below the DNA sequence. Start of introns are labelled EoPI- $X$, where " $X$ " corresponds to intron number. The beginning and the signal peptide, propeptide, metalloproteinase domains and the $C$-terminal extension are specified. Numbers at the right correspond to amino acid numbering of the DNA-deduced pre-pro-PII-SVMP relative to the mature SVMP. The extended $\mathrm{Zn}^{2+}-\mathrm{binding}$ environment (HEXXHXXGXXH) stands on yellow background. The only two amino acids (-124T/A and 15T/A) that distinguish this sequence from that of PI-SVMP EOC00028 (Q2UXQ3) are shown in bold and red. The remains of a disintegrin-like domain and a dimeric disintegrin domain transformed into intron EoPI-12 are underscored in italics and on cyan background. The $N$-terminal glutamine of the metalloproteinase domain has been assigned residue 1. SINE/Sauria, LINE/L2/CR1, LTR/ERV1, DNA/hAT-Ac and DNA transposon retroelements are highlighted on a gray background. Inserted nucleotide sequences in introns 1 (582 nucleotides between positions 1534-1582, including a SINE/Sauria element); 9 (between nucleotides 194-195 of the topologically equivalent intron of PII); 11 (replacing nucleotides 1-66 of PII intron 11 for a stretch of 3281 nucleotides); and 12 (after nucleotide 999 of the homologous PII intron) are underlined. Simple sequence repeats (SSR, microsatellites) are shown in light green background. 
ATG ATC CAA GTT CTC TTG GTA ACT ATA TGC TTA GCA GTT $\pi T$ CCA TAT CAA $G$ gta aga tgt tct gtt tag ttc cct tgt Sp35_Eo Fw

$\begin{array}{llllllllllllllllll}\text { M } & \text { I } & \mathbf{Q} & \text { V } & \text { L } & \text { L } & \text { V } & \text { T } & \text { I } & \text { C } & \text { L } & \text { A } & \text { V } & \text { F } & \text { P } & \text { Y } & \text { Q }\end{array}$

I

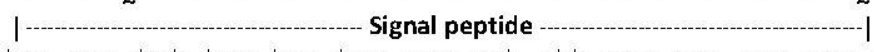

tca gaa tct tac tgc taa aag act att gca $\mathrm{ccc}$ aaa aga ctg cta tgt tgg tag ttt tgg ttt tat tt tga caa tta acc aaa gtt tac ttc act tca gtt tct aaa gat taa gca aaa gaa tgt tct caa gga tca cat ttg ttc taa agt tac ata aat ggt tta ttg gta tt gtt taa att ttc tca agt aag gag caa atc cta agg aaa aaa gtc cta aga ttt tca tta aaa aag cac att cat gtt gga ggt aat ttt ttc caa tga taa gat taa cac att gaa gag gac aga gag aaa gtg ttt gtt gca aaa aaa att cag aaa gca aaa gaa aaa aat gtt tt att ttt aat ttt ctt tca ata ggt caa tta gac atc ctg aaa tta agc atg cat aat att tag cca ggg aca caa tgg ctc agt agg ttc gga taa tga att tgt taa cca gat ggt gag cag SINE/Sauria $\rightarrow$

act ggc ggg tca aat $\mathrm{ccc}$ aag tgc cac gta aca gag tga gtg cct gtt act tgt ccc agt ttc tgc caa cct agt aat tca aaa gca tgt aaa aat cca agt aga aa ata agg acc act aca gtg gga atg taa cag cat tct atg tgc ttt tgg cat CTA GTC ATT CCG GCC ATA TGA Intr1F1PI Fw

cca ctg aga tgt cct tgg aca aac act ggc tct ttg act ttg aat gga gat gag cac caa ccc cta gag ttg gaa atg agt agt atg cat gtg tgg ggg aac ctt tac ttt tac cta ata ttt agc caa ata aaa gct agt gct ttt gat gtt aca gag aat ct cag act gta ctc aat ata atg tga cag tag ctt ttc tca atg caa gat tcc gta tta tct taa agt att tct ttc ctc ctt $\mathrm{ccc}$ tet tac tct gca aaa aat aga gea gtc cct tct gaa att ctt ccc aag ttc tcc ttc tgt gag cta aga tac att tta cat gcc agt tag cca atc tat ggc tat tct tcc tgt atc cca agt cat tcc cac ata tca tta caa aca aga ata ctc aat aga ttc aga agt tgc caa aca aat tta agg gtg aga ggc tca ctg cag tag ccc tgt ttg ttt gtt tgt ttg ttt gtt tgt ttg ttt gtt tgt ttg ttt aaa tag caa aga aag atg aga atg ccc tta tca gga gta att tat ggg gaa caa tgt agg aaa gaa gaa agg gag atg gaa gag aaa ttg ctg ctt aat ctg tgc tct aag caa gag agg aat agt aag ctt gct agt agg tgg aag tga acc cct ccc atg ttt ctg gga gaa tca ggt aca aat agg tag gta cat caa tag ata taa gga aga tgt att ctt ctg tct ggg get gCC CAT CAG TCT GAG AGG ATG CAT TTC Cag ggt aaa Intr2F1PI Fw

atg ctg gtt tct ctg ttt ctc cet ctc tcc ctc tct ctg ttt ctc ttt cac tct ctc tct LTR/ERV1 and DNA/hAT-Ac $\rightarrow$

ctc tct cac aca cac aca cac aca aac aca aac aca cac aga cac aga cac aca cag aga gag aga gag aga gag aga gag aga gag aga gag aga gtc tgt ctc tct agc tcc ttc cct ctc ttt ctc tct ctc tct gtc tgt ctc tgt ctc aat tgt tta ggt gtt tgg gta gca gtt tct ttc cta taa ggt aaa ggt aaa gtt tcc ccc ata cac acg tgc tag tca ttt cca act

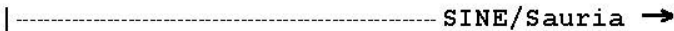

cta gga get gat get cat cgc cgt ttc aaa get gaa gag cca gtg ctt gtc cat gga cat ctc cgg gat cat gtg acc agc atg act aaa tgc cag aga tac atg gaa aac tgt tat ctt

Figure A2. Cont. 
ccc act gca gta gtc cet att ttt cta ctt gca ttt tta tgt gct ttc aaa ctg cta ggt ggg cag aag ctg gga caa gtt aac aag tta act cac tct gtt acg ctg cac tgg gga ttc aac cca cca atc tgc cga cet tct gac cga caa gct cag tat cet aag cta ccg tgt ctc ttt tct ttc cta tac cct gtg gtt att gat att tga att tat tgt ttt gtt tct gct tgt aat tat gag cac aac ata ccc tgt cct ttc cat taa tct gct aac aaa tct tgg tct cag ttc tga ttg tac atc caa gca tac ctg cag gtg tca gac tgc ctc att tac aat ata agt taa aca aca at atc aGT GAC CAT GCA ATG TCC ATA TGG gga CCa act cta aat tt tca Intr3F1PI Fw

cta ttg gtc taa ttc tga atc att gaa tta tgg atg tta tta gat aat tat gat gct gtg aac tct cca tag att att tgg aaa gtg aaa gaa aag aaa gtc agc aaa agt att agg ata gca gta aag tag cat ttc agc tga aag gaa ttc caa ata atc tgc att tgc ata gaa atc taa gtt tgc cta ggt ttt aga cta atg gat atg cta aac cat aac aca ata tgg ttt act gaa ttg tat gaa tca att att gtg att tac agg ttc tca tct tat cac tca ggg cac aaa ctt gca cat tgg ttg aaa tct att tat ttt tct aat tat tca agc tct gaa atg ttt cct ttg cac ctg cac aaa gaa atg tct cct gtt aga cta cca ggt ctc aga cat cat gca gca aat tgc att CCC aga gaa aat ctg gat ttc tgt gct gtg tcc cac tgg gaa aag att cca ttc ttc act gat aac tga ata aca aat tgt gtt gtg gat gtg aaa tcg ata gaa taa cat CCC aaa gaa aac cga atc ttc tet tct ttt tcc tta tga ag GG AGC TCT AAA ACC CTG

AAA TCT GGG AAT GTT AAT GAT TAT GAA GTA GTG AAT CCA CAA AAA ATC ACT GGG TTG CCT $\begin{array}{cccccccccccccccccccc}\text { K } & \text { S } & \text { G } & \text { N } & \text { V } & \text { N } & \text { D } & \text { Y } & \text { E } & \text { V } & \text { V } & \text { N } & \text { P } & \text { Q } & \text { K } & \text { I } & \text { T } & \text { G } & \text { L } & \text { P } \\ \text { GTA } & \text { GGA } & \text { GCT } & \text { GTT } & \text { AAG } & \text { CAG } & \text { CCT } & \text { GAG } & \text { AAA } & \text { AAG } & \text { gta } & \text { aga } & \text { tat } & \text { ttc } & \text { ttt } & \text { cat } & \text { caa } & \text { caa } & \text { att } & \text { att }\end{array}$ Met15PI Fw

ttt tgt cag tcc ata gaa got ttg ata ttc ctt tcc tgc cat tta atg gtt att tgg att ttt cat tgc aat cca tgt tcc tgt ttt att tat tta tet gtt tgt ttg ttt gtt tgt ttg ttt gtt tgt ttg ttt gtt agc aaa ttt tac tgc ctc cca gtt tac aca aaa ttg agg aga atc ttg gca act tac aaa cag aat ttc cta ata tta ata gtt tag tat aac taa tca agt gtt ggg aga aag cca gcc ata cag tga aga gga atc aag cag tga aat aac cat tag tca ttt cat act gca aca gat cca gca aag aca tcc tct $\mathrm{ccc}$ tcc tcc agg aaa tcc cat taa agt tta tct gat ctg cag aga atg gaa gca gtc tag gga gag tcc tgt att aca ggc aaa aaa taa taa act get taa ttt aaa ctt tgt ata tct aca tgc gtc aca tag aaa tat aaa aga tat att tct aga tat gat agt ata gtg gtt aca gct aaa gat tag ttc tat ata cca gat tca tgt ctg gcg tgg gac gac ttt tga gtc aat ctc tct gtc cca gat caa cct cat tca gca ata tgt tca gtt aga gaa tga gat ctt gaa cct atg cag gaa aaa taa ata aag atc tta tca ttc aaa gca cca ggt gaa ata cct aat aat aat aat cta ata aat aat ttg act atc aca aat tat tca tta gat tta aat taa tac aga tgt aga gta tta aga aat gac aca ttt tat ttg cct aaa ttt tga gaa gta aaa cat aaa ctc ttt gtt ttt cag TAT GAA

GAC GCT GTG CAA TAT GAA TTT GAA GTG AAT GGA GAG CCA GTG GTC CTT CAT CTG GAA AAA Prodom 2 Fw/Prodom 2 R

$\begin{array}{lllllllllllllllllllll}\mathrm{D} & \mathrm{A} & \mathrm{V} & \mathbf{Q} & \mathrm{Y} & \mathbf{E} & \mathbf{F} & \mathbf{E} & \mathrm{V} & \mathbf{N} & \mathrm{G} & \mathbf{E} & \mathrm{P} & \mathrm{V} & \mathrm{V} & \mathrm{L} & \mathrm{H} & \mathrm{L} & \mathbf{E} & \mathrm{K}\end{array}$ AAT AA gta tgt taa ctc aga att ttt ttt aac ttt act aaa caa tgt gga aaa tgt ata N $\quad \mathrm{K} \quad$ । EoPI-3 $\rightarrow$

ttc ctg gac aca atc tga gag aaa taa taa ttg cat tcc ttt gtt tgg aaa tta aaa tta aat taa atg tta cta tat aga aaa agt gga tat aga tat taa gta tgt taa tta tgt ctt acc atg aaa ctg aat ttt ttt tac tgc tgt ttt cet atg gaa cat tgc tga acc ata aca at tta gac tca gtt gaa cca ttc aag get cat aac ctt cta tca ctt gaa tta act agg tta gtt atg aaa tat tgc agt gat ttc aaa atg ttt gtt tat get gct tca tag aca ctc cat caa tct gaa taa aat ttt cta tga gac ctg tag agc tgt tca acg tac ata aaa aaa att ata tta aat cat aaa agg cag gaa atg tca tct cct att aag acc cga aag gga cag gac cta aat aac cet gga ttt cac aag cct ttt tta aaa gaa tgc ctt gtg agt ctt ctc aga gtt gtt cca ttt aaa ctg taa atc aag ata tta ata aaa ata tta tac aaa tac gaa cca caa aaa tgg aat gaa att att ttt cag cac aaa aga caa aca tag ttg gaa ggg acc LINE/L2/CR1 $\rightarrow$

ttg gag gcc ttc tag tcc acc ccc tgc tga agc agg aga cta tat cat tcc atg ggt gtc caa ttg ttc ctt gaa aac ttc cca cag ctt ctg aaa get acc cat tcc acc gat taa ttg ttc tca ttc tca gga att ttt ttt taa ttc tag gtt gaa ttt ttc aca ggt aaa taa gcc aca aac acc cct aaa aga aag tgt gcc att aac att acc cta aaa act gta aat gtc ata aca cag ttt aaa tta gtc tgg cag tca cta aac tgc aaa cac gta cct cag cct ggt tca gtt taa tac tat agt taa GAC TGA GAT ACA GAG TTG GTG Cat tgg gaa aga ctg cet gCC

\section{Intr3 Rv}

ttt gat ttt agc atg tat ctt ggt ttt gat cat tct gca tgt acc ttt aaa agt ata ttg tct tta tta tta aac ata tgc tac aag aaa ttg cac tat atg tta gta tct gtg tgt cac ttc ctc cta ttc cac cgg aga tat ttc atg cta aat tct aat gtg tgc ttt aga cgt tct agt gtc $\mathrm{CCC}$ ttt tgt ttt gtt taa ata tga agg cta tac aga aat tca agg gta gca tat gga tgg tct tct ttt gtc ata gaa gca aag cag ggc tac agg ggg aag acc agc aag gat ttc tag att ggc aaa aat gga aga cga tga caa gtt tat tt tct gac atc agg act gga atg tga ctc cat ata ctg gtt taa agc ttc aaa ata tat ctt tct gta tga atg ttg aag gca taa att tcc ttg agg ttg ctt cat ttt act ctt tag taa ata gaa cta tat cgt tct ctt tta gag aca atg cag ttt cat cag att gtt gca tag act aaa aga ata aaa gga aga cgc aat aca aac tgt cct ctc ttt gtc cat cac at cct ctg agc att gat gaa gag aat tgc aca get tca ttt ggt gaa tct agt atg cac tgc ctt cet ctt aaa aag cca tgg gat aga aat acc tgc tcc att ctt ttg ata cca aag aag ata ctt aaa ttg cca tta tac ggt

Figure A2. Cont. 
tgt ttt aaa ctt ct ata aaa tta tct ctg tgc tac ata at cet gat ata gat atc tct tet ttg cat tet ttc cag A GGA CTT TTT TCA GAA GAT TAC AGT GAG ACT CAT TAT TCC Pro2 Fw/Pro3 Rv

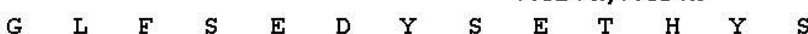

CCT GAT GGC AGC GAA ATT ACA ACA AAC CCT CCT GTT GAG gta ggg tct cac ttt tat gag

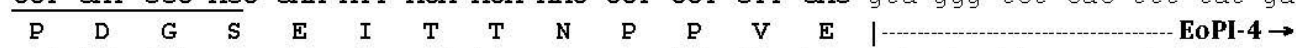
cet ttt tt aag gaa gta aat tga aac aaa tgt ttg tgc act ata tta caa ata tac aag aat gag acc agg cta ctc aaa caa agt gta tat aag tat aaa gta tct tat att gat atg tac tta caa aga tgc ctg gat tgt taa tcc ttg gtt aaa agc caa cat att tgg gag gtg agt ttc aca aat aga ttt att atg aga aca tca ggt ttg taa gat tat att ttc att tt aaa cca gac tac agg gat aaa tgc aaa gtc ttt tat ctg taa tac caa aag tga taa caa ttc act ttg ctc cta tac aga aat cca ttt aac atc tt cat att aaa atg gtg cca aaa atg get cta tca gag gtt aaa aaa tta cag cac taa tat gct tca tgt tgg ctc cat ttc $\mathrm{CCC}$ aaa ttg att taa aag tgc att ctg tgt cta ttt ctg gtt tag cat ctt cat ggg ttg cac aaa tta ctc ctt tgt $g c c$ atc agt ggc act ctc cca tag tgt gac ttg att tat gga gac ttg cat tta tcc tat gtt cct tt gca ata gtc agt at aag aag gtt ttc tgt cct cct gaa tca aaa tt tct gga aaa ctg ctg tct aaa tat ttc att gat gtt atg gaa tac att gga act gta ctt ctg ctc atc aaa tca caa tac aaa gcc tta acc agt gta gtc ctc TTG CCT CCC TAT AG GAT CAC TGC TAT TAT TAT GGA CGC ATC CAG AAT GAT GCT GAC TCA

\section{IntrB13-1 Fw/Met16PI Rv}

$$
\begin{array}{lllllllllllllllll}
D & H & C & Y & Y & Y & G & R & I & Q & \text { N } & D & A & D & S
\end{array}
$$

ACT GCA AGC ATC AGC ACA TGC AAT GGT TTG AA gta aga tag tct cta atc ttt tat ttg $\begin{array}{lllllllllllll}T & A & S & I & S & T & C & N & G & L & K & & \end{array}$

ttt att aat aat aat ata gtg ctc ttg gag ttc taa ttg tta aaa tga agg aca tcc tca gtt ttt cat gga aat tag ttg ggt gtg atc cag gat ttc ggc aga att aag aca tac tt ggt tga aaa cca aga aga gct gct gcc agc cag gag aaa aac tat gga gct aaa tca cat aag tct aaa gga gct tcc aag ccc cgg tct cct ttc cca ggg tga ggt gat att aca ggt aga gaa gat tag tag gtt tca aat tgg aga cct tgc tag aaa gtg tac agg aag agg caa gaa gtt tca gtt cta ccc aga aac act ttc ttg agt cac tct gca cac tet ctt cag cca act aga tat gtt aac tac ata aag atc cca gaa ttc aga agg tcc cta tca ata gta aga atg aac atc acc tca aca tct ttt act gaa aaa aga cac tga aac tca cct ttg aac aga gac tgt gtc cat gga gtg gag gaa taa atg aaa agc tgg aac aga gca gaa taa caa cag aaa aat aaa gga aaa aca gaa tga cag aat aat agc att gga agg gac ttt gag gtc ttc

$$
\text { LINE/L2/CR1 } \rightarrow
$$

tag tcc aac ttc ctg ctc aag tag gag acc tat atc atc cta gac aaa tag ctg tca atc ttc tct taa aaa gca gta gtg atg gaa cac cca caa tgt ctg aat agg tta att gtt cca ttt gtg aga aaa tta ctc ctt agt tct aac tta ttt ctc tct ttg gtt act ttc cac gca ttg ctt ctt ctc ctg cca tca ggt gaa gaa tag gtt gtc cca cat ttt tta tga cag cct ctt aaa tac tta aag att atc aag tca tct cta ccc ctt ctt gtc act agc atg agt ata ctc att gtc tgc agc cat tct aac ctc cag tta gta tgc att ctt att cct tca ttg tta ctc ctg ttg ttc tgc att gac ttc tct atg aag atg ctt gcc aag aat tct tat ttt cat tat tta tta aat atc ctg gtc atc ctg act ctt atc tta aat tgc tat caa act aat ctg att tta ttt cet tga cca cag aca aat att gtt cta tac ttg ttt aaa gta aat tgc agt att acc tat aac tct ttt tag ata ttt tag cag tta tat ttt tcc ttt ttt atc cta ctt agt tgt gat tct tga get tta tca gta ata tat atg ata aat ata aag tat ttt acc ctt atg aaa taa agt ttt aca caa agc aga atg tta caa ttg gct tta gtg ttg tat tta tgt agc tag aaa ctt att ttt tta aca tcc tgg aaa tat aca ata ttg ggt tcc atg cca aaa tat ttc caa aca aaa ctg tac acc tat ttt gtg get gca ctg agt ttg tga aat ctc tca tat ctt tct gat cat aac tgc atc tat gaa aag tat gag aaa gtg att tga gtg ctg agg aaa gaa tat aa ata ttc act cat tgt taa gaa gga att caa aaa cat gag gtt agt tga aaa tgg gtc tca gag $\mathrm{ccg}$ agt ttc att acc caa cta ggt aac atc atc agt gca gtt tt ctc tga act aac aat att ctc ttc tet tgc ttc tcc atc tct gat cat cet tet cac att gtt tta cag A GGA TTT TTT ACG CTT CGT GGG GAG ACG TAC TTA ATT GAA CCC TTG AAG $\begin{array}{lllllllllllllllll}G & F & F & T & L & R & G & E & T & Y & L & I & E & P & L & K\end{array}$ GTT CCC GAC AGT GAA TCC CAT GCA GTC TAC AAA TAT GAA GAT GCC AAA AAA AAG GAT GAG Prodom 1 Rv/Prodom $1 \mathrm{FW}$ $\begin{array}{cccccccccccccccccccc}\text { V } & \text { P } & \text { D } & \text { S } & \text { E } & \text { S } & \text { H } & \text { A } & \text { V } & \text { Y } & \text { K } & \text { Y } & \text { E } & \text { D } & \text { A } & \text { K } & K & K & \text { D } & \text { E }\end{array}$ $\begin{array}{lllllllllllllllllllll}A & P & K & M & C & G & V & T & L & T & N & W & E & S & D & K & P & I & K & K\end{array}$ GCT TCT CAT TTA GTT GCT ACT TCT GAA gta agt ctc ata ata aac ata gtt taa gat tac

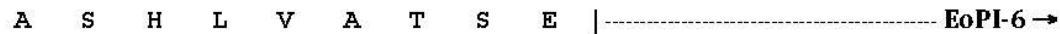

ata cta att tcc ttg tct tga aaa tat aaa gta aga gag aat ttc ctt tgg gaa ggg gtg ata gat aga att caa aag gga gaa gcc ccc att tct ata ttt tta ttg tag cca tgg cat aaa aga aag aat gga aac ttg agg aac aga aa tac att ttc cag gct tat agc att ttc ttt ggt cat tca aac tta gtt tag aga ttt gaa tca aaa tct att taa atg agt ttc taa att atc tct agt ttc taa gtc aat gtt gaa aag taa tta aat tat caa ttt gga ttc ctc ttt tat gca tgc aga gag gat ggg gga caa agt ggt ttg aaa tat taa atg gtt tta aga tgt ctg ata agg cca tta cat aat tgt tac tcc att atc caa tet gat ttg aat cat caa gtt gga ttg atg caa tga atg gat gaa aag tga caa tgt gaa cct agt cac aat tga ccc tta tgc tct caa tat ttt cct cct tta ttg gac gca caa aaa tta gaa aac aaa ata ttg cat cca aag tga cag ttc ctt tcc atc ttt ttg gtg gca aaa gtt gaa act ggc tga aaa atc tct act gtt tt att aga atg tta aaa ttg aca TGG AAC AAC AGC TGT TGT TAT GAC IntronB7PI FW

Gga ata cca aaa cac aag tga aga cgc caa atg aag cet ggt ttg tct ttt ggc ttc ttt cat tct ggc aat tca aga ttc ttt atc ctc agc aat ttg tgg tta tac gtt aca ttt aac

Figure A2. Cont. 
tgg tcc tta att ggt tct cta tct tga atg ttc ttg tct caa agt cag tca tga tca ctt tat tcg gac tcg atc ttg tgc agc aga att aag aaa gtg gct gtg aga ggg gaa gga gag aaa ttg cat tct aga att gca act tgg cct ggt agt tct gta cac tt cat agg gaa aga agc aat taa tgc aca ctc aca cat tca gtc tta gca aaa gtg tac ttc ctg agc aac ctg ggt act cta gtg gag gaa tct ccc ttt gga atg cag gaa gga ggg gct ctt tcc tag aga aat aag agg cag atc aga aat gaa tcc ttg gat tgc aga aag atg ccc gga agg gat cct gcc taa agc ctt cac agg atg tat ctc atg gtt aca cag agt gtg ctt tag tct ggc aag gtt cct gtc tac agg aga aga gca ata aag aaa cta ctc taa gta att aac cac agt ttc ttg ttc ctt gtg ctc aag aag tct gaa aca ata ttt cag taa tca ttt taa aat tac atc act gaa aag aca taa ttc ttt ggc cat taa gag tga gtt tag tgg gtc aaa atg tct att tgc ttc tcc tta cct tgt ctt tga tgc ata gtg tga tat gat tcc agg cca ggt cta att gCa tGA CCA CAT CAG CAT GTC TCT CAC gtg att ggt tgg cat cet ttg ttt gag aag ggg

\section{IntronB7PI Rv}

aag gaa agt tga gaa agt cat tga agc atc att ttg aca ggg tga aaa aca ggt caa aga gaa cag ttt cet cat atg cct att aaa ttc ttt tca gag tta ggt att cat ata tac cat tat ctt gac aat cca ttg aat aac gta ctt ttt tct tca aaa ctt tgt cag cac ttt ctc taa gtc aga gtt $\mathrm{CCC}$ aaa cct ttc cag ctt tgg gga tag gtg agg gag agg gga tgg ttc cac atg aac agt ggg gtc agg tgt gta ccc agc tct att tgt gcg agc agt ggg cac aca tac cca ctc gtg taa aca gaa cac att cac cta tgc ttg ttc act ggt cat aca agt aga gat gca gct gct cac ctg cca ttt cca tgg ccc agt tct aaa ggg atc aag gcc cag ggc taa aac tct aac aa ctg tct ccc tgt aaa tat ctt ctt gaa aga act gat att tct gga agt tga cca gag agt aaa aca agc att ttt ctg att atc tga ggt tga cca aca ttc ttg ttg gct get ggg agt aac atg ttt aaa cat cca ttt tt aat tgt tct gtt tag CAA CAA

CAT TTT GAC CCA AGA TAC ATT CAG CTT GTC ATA GTT ACA GAC CAC GCA ATG gta agt atc Met4 PI Rv/ Met4 PI Fw

$\begin{array}{llllllllllllllllll}\text { H } & \text { F } & \text { D } & \text { P } & \text { R } & \text { Y } & \text { I } & \text { Q } & \text { L } & \text { V } & \text { I } & \text { V } & \text { T } & \text { D } & \text { H } & \text { A } & \text { M }\end{array}$

tta aat acc ttt cca tet act ttc tgc att gag tac agc att ttg ttt ttg aat ttt tta atg cgg gca ttt ttc aag aga tta ata cac atc cta act gac ttt ctc tta taa tgt gcc tta tca taa act ttg ata ttt ttt gtt att gat cca acc aat att tac aaa gtt aag agt cat ttg taa ata tat tct aat ttg cac att tt tat ttc tgg ata cca tct aag ttt att ttt atc cca cta tag tta aaa att tct gta ggt ttc ttt gaa atg ttc cca cac tct tt ttc atc caa gct ggc aac aca cac aca cac aaa aaa aga tgt aca gtg aag aat gct ttt ctc aca cat ctc gga aca tgg aaa tat ttt ctc caa cca aaa aaa agg ccc atg tat gct gct gaa aag tta aaa atc tta ata tat taa cgg cac aaa tgt aga tta aaa aat cag cac aaa aga att att tgg act get tgg ata aaa att att aca tca aca aat tca aag atc ctt tca cta ata tot tct tet tct cct ccc ttc ttt ccc ttc tta taa tta tca act tgt ctt aac tte tet tet tet tgt ag GTC ACG AAA AAC AAC AAT GAT TTA ACT GCT ATA ACA ACA

Met8PI Rv

TGG ATA CAT CAA ATT GTC AAC GAT ATG ATT GTG gta aga aca aat get tgt tca ttt taa

$\begin{array}{llllllllllllll}\text { W } & \text { I } & \text { H } & \& & \text { I } & \text { V } & \text { N } & \text { D } & \text { M } & \text { I } & \text { V } & \longrightarrow\end{array}$

act tca ctt agg $\mathrm{CCC}$ agc cga gat ttt gat tgt gtt aag ata aca aac tta atc agg taa aga aag tag atg gat ttc taa atg aat ggt ttc ttt tgt ggc ttt gag tga tgt aaa act aag tta ttt gac cag ctg gtg atc cag cat gtg agg cca tcc cag aaa atg ggt taa ttc agt aac taa att aat aag cct cta atg gaa ata gag tca ttg got tgg gag gat gca aat cca aaa gtg ttg caa gaa ttc agt cag aag tat acc tgt agt tgg att ctg tca ggg ctg tgg tta atg gtt ctg aac ttg gag att aga aag tga tgg aca gag tca gtg tca ggc gcc gtg cct gac aca tgc act ggg ggt gga gga gtg ata gcc gcg cgc ccc tcg ttc att gga ggg gcc ggt aca agg acg cgg agc tcg ctc gca gct gga ttg tgg cgg caa ggc aac tga cag ctg cag agg gtg gag gcg gcc ttt cca gtg gct ggt gga gac tgc gga aga gag gag acc ggc gga caa act aag cca cag aac ttt gga gtg gtt cca gca cag aag gag gag cca cga ggg Cgg TGT GCT TAC CCA ACA CTG AGC Ctg gaa aaa agg acg ata tgt tta cgt ttg Intron B16 Fw

get get tgg agt tca ttc cCC CCC cet tgt ctg cca gat get get gcg ggg ctg tat tt ggg tgg tgt tag gga ggg gct agg ata ggg gta aag get aag tgt gga gaa ggt gat ggg aat aca tac ttg gtg gtg gat tag gaa gaa ggg cgg gtg aaa tga agg gtg gtg att ttg tgt cac cat gct ggg agg ggt gga gcc cag gcg gaa ggg tgt gge tgg gtg tag caa tgt att taa tag tot ggo atg gat atg taa gcc aac gct ggc tt ttc cca ctt cta cot tga gtt ttg ttg ctg aat aaa gcg tta ttt ctt ttt gga tac ttc ccg tgc ctg tga gac tgc tca ttg gtg agt aac tgg acg gga ggg act gac agt cag cct att cca agc tca gtt gat tat gaa aat gat ctc tga gaa gaa acc ttg aga gaa gaa ctc aat ttg act tgg att gtg gta ctt agg agg aga tac agt cat act gaa ttc tct gat tag cct tat tta cat tt cac tat tga tga tca gtt aga aac agg gag aga aca gga aga ttg agg aga taa ctc tcc tgt tag tcc acc tac tct tct get cct get tac aga aaa taa tca cct get tct tet tat cat gtt att tat ttc tct tgc tta ata cat tgt tat tat tcc ctg ata tat aga gtc ctt agt act atc tca get tgg tgg tta cct tgc aga cot ttc att att tga gta ctc tac atg aat aac caa aca gca agc tca gag cac caa gga ctt caa cct tga gct tct att gat acc tag gtc tat ttt ttt tt taa atc tac aaa aag agg cca atg tca gtt gct ttt taa tat tga aat gtt tct caa ggt tta ctt tgt ttg agt ttc tga ttg atg cca tgt cca cct gac ag ATG TAC ATA GAT TTG AAT ATT CAT ATA ACA CTG GCT GCC GTA GAA ATT TGG TCC AAT GGA $\begin{array}{llllllllllllllllllll}M & Y & \text { I } & \text { D } & \text { L } & \text { N } & \text { I } & \text { H } & \text { I } & \text { T } & \text { L } & \text { A } & \text { A } & \text { V } & \text { E } & \text { I } & \text { W } & \text { S } & \text { N } & G\end{array}$ GAT TTG ATT ACT GTG ACA TCA TCA GCA CGT GAA ATT TTG AAC TCA TTT GGA GAA TGG AGA 
tgt ttt aaa ctt ct ata aaa tta tct ctg tgc tac ata at cet gat ata gat atc tct tet ttg cat tet ttc cag A GGA CTT TTT TCA GAA GAT TAC AGT GAG ACT CAT TAT TCC Pro2 Fw/Pro3 Rv

\section{$\begin{array}{llllllllllllll}G & L & F & S & E & D & Y & S & E & T & H & Y & S\end{array}$}

CCT GAT GGC AGC GAA ATT ACA ACA AAC CCT CCT GTT GAG gta ggg tct cac ttt tat gag

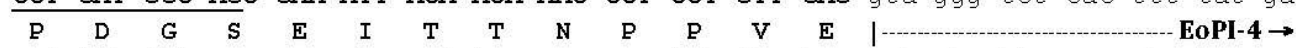
cet ttt tt aag gaa gta aat tga aac aaa tgt ttg tgc act ata tta caa ata tac aag aat gag acc agg cta ctc aaa caa agt gta tat aag tat aaa gta tct tat att gat atg tac tta caa aga tgc ctg gat tgt taa tcc ttg gtt aaa agc caa cat att tgg gag gtg agt ttc aca aat aga ttt att atg aga aca tca ggt ttg taa gat tat att ttc att tt aaa cca gac tac agg gat aaa tgc aaa gtc ttt tat ctg taa tac caa aag tga taa caa ttc act ttg ctc cta tac aga aat cca ttt aac atc tt cat att aaa atg gtg cca aaa atg get cta tca gag gtt aaa aaa tta cag cac taa tat gct tca tgt tgg ctc cat ttc $\mathrm{CCC}$ aaa ttg att taa aag tgc att ctg tgt cta ttt ctg gtt tag cat ctt cat gog ttg cac aaa tta ctc ctt tgt $g c c$ atc agt ggc act ctc cca tag tgt gac ttg att tat gga gac ttg cat tta tcc tat gtt cct tt gca ata gtc agt at aag aag gtt ttc tgt cct cct gaa tca aaa tt tct gga aaa ctg ctg tct aaa tat ttc att gat gtt atg gaa tac att gga act gta ctt ctg ctc atc aaa tca caa tac aaa gcc tta acc agt gta gtc ctc TTG CCT CCC TAT AG GAT CAC TGC TAT TAT TAT GGA CGC ATC CAG AAT GAT GCT GAC TCA

\section{IntrB13-1 Fw/Met16PI Rv}

$$
\begin{array}{lllllllllllllllll}
D & H & C & Y & Y & Y & G & R & I & Q & \text { N } & D & A & D & S
\end{array}
$$

ACT GCA AGC ATC AGC ACA TGC AAT GGT TTG AA gta aga tag tct cta atc ttt tat ttg $\begin{array}{lllllllllllll}T & A & S & I & S & T & C & N & G & L & K & & \end{array}$

ttt att aat aat aat ata gtg ctc ttg gag ttc taa ttg tta aaa tga agg aca tcc tca gtt ttt cat gga aat tag ttg ggt gtg atc cag gat ttc ggc aga att aag aca tac tt ggt tga aaa cca aga aga gct gct gcc agc cag gag aaa aac tat gga gct aaa tca cat aag tct aaa gga gct tcc aag ccc cgg tct cct ttc cca ggg tga ggt gat att aca ggt aga gaa gat tag tag gtt tca aat tgg aga cct tgc tag aaa gtg tac agg aag agg caa gaa gtt tca gtt cta ccc aga aac act ttc ttg agt cac tct gca cac tet ctt cag cca act aga tat gtt aac tac ata aag atc cca gaa ttc aga agg tcc cta tca ata gta aga atg aac atc acc tca aca tct ttt act gaa aaa aga cac tga aac tca cct ttg aac aga gac tgt gtc cat gga gtg gag gaa taa atg aaa agc tgg aac aga gca gaa taa caa cag aaa aat aaa gga aaa aca gaa tga cag aat aat agc att gga agg gac ttt gag gtc ttc

$$
\text { LINE/L2/CR1 } \rightarrow
$$

tag tcc aac ttc ctg ctc aag tag gag acc tat atc atc cta gac aaa tag ctg tca atc ttc tct taa aaa gca gta gtg atg gaa cac cca caa tgt ctg aat agg tta att gtt cca ttt gtg aga aaa tta ctc ctt agt tct aac tta ttt ctc tct ttg gtt act ttc cac gca ttg ctt ctt ctc ctg cca tca ggt gaa gaa tag gtt gtc cca cat ttt tta tga cag cct ctt aaa tac tta aag att atc aag tca tct cta ccc ctt ctt gtc act agc atg agt ata ctc att gtc tgc agc cat tct aac ctc cag tta gta tgc att ctt att cct tca ttg tta ctc ctg ttg ttc tgc att gac ttc tct atg aag atg ctt gcc aag aat tct tat ttt cat tat tta tta aat atc ctg gtc atc ctg act ctt atc tta aat tgc tat caa act aat ctg att tta ttt cct tga cca cag aca aat att gtt cta tac ttg tet aaa gta aat tgc agt att acc tat aac tct ttt tag ata ttt tag cag tta tat ttt tcc ttt ttt atc cta ctt agt tgt gat tct tga get tta tca gta ata tat atg ata aat ata aag tat ttt acc ctt atg aaa taa agt ttt aca caa agc aga atg tta caa ttg gct tta gtg ttg tat tta tgt agc tag aaa ctt att ttt tta aca tcc tgg aaa tat aca ata ttg ggt tcc atg cca aaa tat ttc caa aca aaa ctg tac acc tat ttt gtg get gca ctg agt ttg tga aat ctc tca tat ctt tct gat cat aac tgc atc tat gaa aag tat gag aaa gtg att tga gtg ctg agg aaa gaa tat aa ata ttc act cat tgt taa gaa gga att caa aaa cat gag gtt agt tga aaa tgg gtc tca gag $\mathrm{ccg}$ agt ttc att acc caa cta ggt aac atc atc agt gca gtt tt ctc tga act aac aat att ctc ttc tet tgc ttc tcc atc tct gat cat cet tet cac att gtt tta cag A GGA TTT TTT ACG CTT CGT GGG GAG ACG TAC TTA ATT GAA CCC TTG AAG $\begin{array}{lllllllllllllllll}G & F & F & T & L & R & G & E & T & Y & L & I & E & P & L & K\end{array}$ GTT CCC GAC AGT GAA TCC CAT GCA GTC TAC AAA TAT GAA GAT GCC AAA AAA AAG GAT GAG Prodom 1 Rv/Prodom $1 \mathrm{FW}$ $\begin{array}{cccccccccccccccccccc}\text { V } & \text { P } & \text { D } & \text { S } & \text { E } & \text { S } & \text { H } & \text { A } & \text { V } & \text { Y } & \text { K } & \text { Y } & \text { E } & \text { D } & \text { A } & \text { K } & K & K & \text { D } & \text { E }\end{array}$ $\begin{array}{lllllllllllllllllllll}A & P & K & M & C & G & V & T & L & T & N & W & E & S & D & K & P & I & K & K\end{array}$ GCT TCT CAT TTA GTT GCT ACT TCT GAA gta agt ctc ata ata aac ata gtt taa gat tac $\begin{array}{llllllllllll}\text { A } & \text { S } & \text { H } & \text { L } & \text { V } & \text { A } & \text { T } & \text { S } & \text { E } & \text { EoPI-6 }\end{array}$

ata cta att tcc ttg tct tga aaa tat aaa gta aga gag aat ttc ctt tgg gaa ggg gtg ata gat aga att caa aag gga gaa gcc ccc att tct ata ttt tta ttg tag cca tgg cat aaa aga aag aat gga aac ttg agg aac aga aa tac att ttc cag gct tat agc att ttc ttt ggt cat tca aac tta gtt tag aga ttt gaa tca aaa tct att taa atg agt ttc taa att atc tct agt ttc taa gtc aat gtt gaa aag taa tta aat tat caa ttt gga ttc ctc ttt tat gca tgc aga gag gat ggg gga caa agt ggt ttg aaa tat taa atg gtt tta aga tgt ctg ata agg cca tta cat aat tgt tac tcc att atc caa ttt gat ttg aat cat caa gtt gga ttg atg caa tga atg gat gaa aag tga caa tgt gaa cct agt cac aat tga ccc tta tgc tct caa tat ttt cct cct tta ttg gac gca caa aaa tta gaa aac aaa ata ttg cat cca aag tga cag ttc ctt tcc atc ttt ttg gtg gca aaa gtt gaa act ggc tga aaa atc tct act gtt tt att aga atg tta aaa ttg aca TGG AAC AAC AGC TGT TGT TAT GAC IntronB7PI Fw

Gga ata cca aaa cac aag tga aga cgc caa atg aag cet ggt ttg tct ttt ggc ttc ttt cat tct ggc aat tca aga ttc ttt atc ctc agc aat ttg tgg tta tac gtt aca ttt aac

Figure A2. Cont. 
$\begin{array}{llllllllllllllllllll}D & \text { L } & \text { I } & \text { T } & \text { V } & \text { T } & \text { S } & \text { S } & \text { A } & \text { R } & \text { E } & \text { I } & \text { L } & \text { N } & \text { S } & \text { F } & G & E & \text { W } & R\end{array}$ CAG AGA GAT TTG GTG AAT CGC ATA ACG CAT GAT AAT GCT CAG TTA CTC AC gta cgt ctC $\begin{array}{lllllllllllllllll}\text { Q } & R & \text { D } & \text { L } & \text { V } & \text { N } & \text { R } & \text { I } & \text { T } & \text { H } & \text { D } & \text { N } & \text { A } & \text { \& } & \text { L } & \text { L } & \text { T }\end{array}$ EOPI-9 $\rightarrow$ act gtg ggg aat ggg agt tag ggt gtc tga gag tgg agg gtt atg goa agg tta ctg ctt gca tag agc ttc tgt tet atg ctg tat get tga aac cat gca tgt act aca ttt ctg ggt caa agt cag cca ctt ata gat gag agc tgg ctt tga gaa gtc ctt gaa tga tta cag gtg aaa aac ctg gat tta aaa ctc aaa ctt tta tga cta tcc tgc tat act ttt gca gtt ctt ata cat aca ttt taa ttc aat taa ata atg ttt tgg ttc cca ttc tgc acc aag taa atg aac act tgt att ata caa aag cat aca gaa tge tat tac ctt ctc tga aat ggt gcc tat

\section{SINE/Sauria $\rightarrow$}

tta att act tgt gtt tga act gct agg gta gca gga gct gga gca agt aac agg agc tca ttg cat cag gca aaa ctt agg tct caa act att cac ttt cca gtc aac tag tcc act gta tta act get cag ttc cac acc cca tca gcc tat ata ttg tca aac aat tta atg agt gag aaa ctg cta tgt tta gtg tct tat gtc tgt aac ata ctg cag aaa taa caa ttg tgt tgt gag caa tcc tac agg atg ctt gca taa caa gta ggt ctt atg aaa cag gga tta cat tga agg cag att taa gtg caa ggg aga ata tta aaa aaa tac cca cag atg gtg gtt tga ctt gca att caa tca ctc ttc cca aaa cca tct agt cca ata acc cca caa cta aat tga tag aca aaa tct agt aaa taa ata tgt att tgt taa ttt aat gga att aaa tta acc caa atg ggt tga att ctg aca aca tgc taa ccc tct ccc caa cet cat tca tac taa tgc cat cca aac aca act tat agt gaa aaa tag atc ctg aat att ttt aac ggg cet get tag tta aat ggc ttc tga gac tag agc cta aaa tga att cta gca ttc tga aaa ctg gta gta act gag gac agg tcg agg agt tta cag aaa tcc ata ctt atg tat gac cta aga cta cat aac atg ctt cta tat tca att tta tcC CCC tCC cet tct tet tat ag A GCC GTT AAC CTC AAT

GGT GAT ACT ATA GGA AGA GCT TAC ATG GAG AGC ATG TGT GAT CCA AAG AAA TCT GTA GGA Met3PI Rv/Met3PI Fw

$\begin{array}{lllllllllllllllllllll}\text { G } & \mathrm{D} & \mathrm{T} & \mathrm{I} & \mathrm{G} & \mathrm{R} & \mathrm{A} & \mathrm{Y} & \mathrm{M} & \mathrm{E} & \mathrm{S} & \mathrm{M} & \mathrm{C} & \mathrm{D} & \mathrm{P} & \mathrm{K} & \mathrm{K} & \mathbf{S} & \mathrm{V} & \mathrm{G}\end{array}$ ATT AAT CAG gtt agt aga aag gat att cta tta tct att tgt act caa gcg aaa cgt ggc

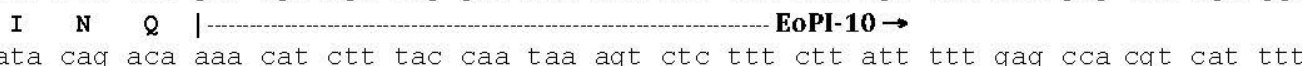
cac cca tat tta ttt gca gat ttg aca tct cca ggt cct gcg tca act aat ggc att ttg aca cag tgc att cta gaa caa gct ttt tta atg caa tga gct ata tgt caa gga tga gaa tat att ata atg ttt atg gtt cag tca aac tgt act ctg att ggc aaa tga aca ggt caa agc atg tta caa cac ttc caa ata atg ctt ctg aac aat agt ctt agc aat ccc aaa gac aaa cat gaa ttc att $\mathrm{cca}$ aga aat tta gtg tct aga ttg cat atg att gaa ttc tag tac att gag aaa aca aaa aaa tac taa atc tac tca aaa aga aaa aaa acc ctc tag tat ttt aag aaa acc ata tta gtt aag tga tgc tat gca ttt att gag aaa gag taa act tag ctt ttt gtt cac ata gaa aga atg gag aga cat ggt aat aaa caa aag tta tac aac aaa act cat aaa gtt ttg ttt ctt aat aag cag agt tag gat cct ggt agt agt agg tac tca taa gcc tac ttg ctc aag aag gtt att tta ttc aga aag agc agc tca ttc tta agt gtg ttt agg atg get acc ttc att att ctg aaa atg taa gct tgt agc aaa gga cac tga gta gtt

ttt ttc aac tga cgt ttc ctg taa gtc agg get gtc aaa ctc aat ttc att gag gga cgc DNA Transposon

atc agc att gcg gtt gcc ctc atg gog gca gtc ggg tat ggc cag ggt ggg cac agc cca cag gca tgg ctg gag tgg gta tgg cta agt ttt agt aac tga ata agt gca cat agc aaa tgg atg cat aca ttt tga tct tat cet gtg ctg tag ctt ctg ggc ttt aaa gtt tcc ttc tgg atg tat ttg tgt atg ttc tgg agt cat ggt ggg cac aca tac ttt cag agg agc tag agg aat cct gag atg gta tcc tca act taa aat tgg tca ctg ggt cac cag ttt agc cac tta gtt cta ata att gga ttc act tta cgt ttc tgg cag agt aac aat aaa aaa agt att ctt att tct tca $g$ GAT CAT AGC ACA GTA CAT CTT TTG GTT GCA GTT ACA ATG GCC CAT

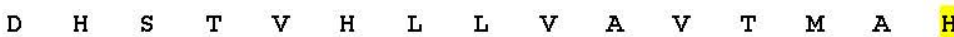
GAG CTG GGT CAT AAT CTG GGT ATG GAT CAT GAT GAA AAT CAG TGT AAT TGT GGT GCT TCC

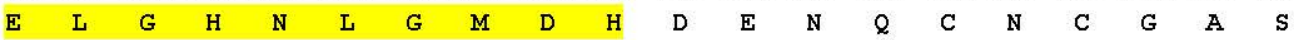
GGA TGC GTT ATG TCT GGG GAG CTA AG gta agt act gag gaa tat get taa tgg gtt ttg

\section{Met2PI Rv}

$$
1
$$

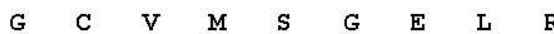
| EoPI-11 $\rightarrow$

aat caa ctt att tt aaa tgg tta caa aaa tga aaa ggt cag ttt agt tac aaa aaa gag gtc att tgg tca ttt gtt tot cac ttt atc atg tgt agt taa aat gtt tca tat tta aaa agg tag aaa tgt ttt cag caa ttg aat agc cat cat tca cag ctt cct cca aaa cac cag aat tta aag aaa cag cat agt aag cag agc caa ata tta ata tct gtg atc aat aaa aga gga aac caa get aaa tgt taa aga ttt tcc taa agt ggg aag cag aat ggg gaa aat tat tct gaa cca ggc aac acc agg aaa aaa aga taa att cgc atc ctt ggg gtt ttc cta ctt tgt cct ccc atc aag gta gaa tat gtg aca tct ctt tca tat act gca gtc ttc tga aat aaa cag ata gca atg tac agg aag tca ttg act tac agt tac ttt gaa gtt aca acg tcc atg aga act atg act tat gac tgg tcc tcg cag tta caa cca tca tag act ccc ttc agc aac aaa atc aaa att tgg gca gtt ggt cac acc caa gtg ggt tca tga tga ctg cat cat cct gta ttc atg gga tcc tca ttt gta cat tcc aag gtg tct tcc cat atg caa agc caa tag acc aaa ctg ggt acc tta aag act gtg tga ttc act taa caa agt ggc aaa act ggt tet aaa aCT GAC TAT GAC TCA CTT AAC AAC TGG ctt cCC ttg gaa agt gga aat tct ggt IntrB23PI Fw

cct agt tgt ggc agc gag tca aga act agc aat att ttc ttc gag gtg aaa gtt tag gcc tta ctt cat caa gaa gga aaa aga aa gga aaa agg aag caa aat ctt ctc agc aat tgt

Figure A2. Cont. 
tgt ttt aaa ctt ct ata aaa tta tct ctg tgc tac ata at cet gat ata gat atc tct tet ttg cat tet ttc cag A GGA CTT TTT TCA GAA GAT TAC AGT GAG ACT CAT TAT TCC Pro2 Fw/Pro3 Rv

\section{$\begin{array}{llllllllllllll}G & L & F & S & E & D & Y & S & E & T & H & Y & S\end{array}$}

CCT GAT GGC AGC GAA ATT ACA ACA AAC CCT CCT GTT GAG gta ggg tct cac ttt tat gag

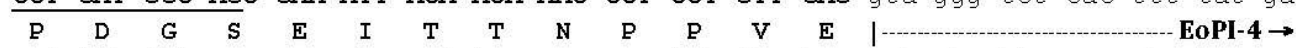
cet ttt tt aag gaa gta aat tga aac aaa tgt ttg tgc act ata tta caa ata tac aag aat gag acc agg cta ctc aaa caa agt gta tat aag tat aaa gta tct tat att gat atg tac tta caa aga tgc ctg gat tgt taa tcc ttg gtt aaa agc caa cat att tgg gag gtg agt ttc aca aat aga ttt att atg aga aca tca ggt ttg taa gat tat att ttc att tt aaa cca gac tac agg gat aaa tgc aaa gtc ttt tat ctg taa tac caa aag tga taa caa ttc act ttg ctc cta tac aga aat cca ttt aac atc tt cat att aaa atg gtg cca aaa atg get cta tca gag gtt aaa aaa tta cag cac taa tat gct tca tgt tgg ctc cat ttc $\mathrm{CCC}$ aaa ttg att taa aag tgc att ctg tgt cta ttt ctg gtt tag cat ctt cat gog ttg cac aaa tta ctc ctt tgt $g c c$ atc agt ggc act ctc cca tag tgt gac ttg att tat gga gac ttg cat tta tcc tat gtt cct tt gca ata gtc agt at aag aag gtt ttc tgt cct cct gaa tca aaa tt tct gga aaa ctg ctg tct aaa tat ttc att gat gtt atg gaa tac att gga act gta ctt ctg ctc atc aaa tca caa tac aaa gcc tta acc agt gta gtc ctc TTG CCT CCC TAT AG GAT CAC TGC TAT TAT TAT GGA CGC ATC CAG AAT GAT GCT GAC TCA

\section{IntrB13-1 Fw/Met16PI Rv}

$$
\begin{array}{lllllllllllllllll}
D & H & C & Y & Y & Y & G & R & I & Q & \text { N } & D & A & D & S
\end{array}
$$

ACT GCA AGC ATC AGC ACA TGC AAT GGT TTG AA gta aga tag tct cta atc ttt tat ttg $\begin{array}{lllllllllllll}T & A & S & I & S & T & C & N & G & L & K & & \end{array}$

ttt att aat aat aat ata gtg ctc ttg gag ttc taa ttg tta aaa tga agg aca tcc tca gtt ttt cat gga aat tag ttg ggt gtg atc cag gat ttc ggc aga att aag aca tac tt ggt tga aaa cca aga aga gct gct gcc agc cag gag aaa aac tat gga gct aaa tca cat aag tct aaa gga gct tcc aag ccc cgg tct cct ttc cca ggg tga ggt gat att aca ggt aga gaa gat tag tag gtt tca aat tgg aga cct tgc tag aaa gtg tac agg aag agg caa gaa gtt tca gtt cta ccc aga aac act ttc ttg agt cac tct gca cac tet ctt cag cca act aga tat gtt aac tac ata aag atc cca gaa ttc aga agg tcc cta tca ata gta aga atg aac atc acc tca aca tct ttt act gaa aaa aga cac tga aac tca cct ttg aac aga gac tgt gtc cat gga gtg gag gaa taa atg aaa agc tgg aac aga gca gaa taa caa cag aaa aat aaa gga aaa aca gaa tga cag aat aat agc att gga agg gac ttt gag gtc ttc

$$
\text { LINE/L2/CR1 } \rightarrow
$$

tag tcc aac ttc ctg ctc aag tag gag acc tat atc atc cta gac aaa tag ctg tca atc ttc tct taa aaa gca gta gtg atg gaa cac cca caa tgt ctg aat agg tta att gtt cca ttt gtg aga aaa tta ctc ctt agt tct aac tta ttt ctc tct ttg gtt act ttc cac gca ttg ctt ctt ctc ctg cca tca ggt gaa gaa tag gtt gtc cca cat ttt tta tga cag cct ctt aaa tac tta aag att atc aag tca tct cta ccc ctt ctt gtc act agc atg agt ata ctc att gtc tgc agc cat tct aac ctc cag tta gta tgc att ctt att cct tca ttg tta ctc ctg ttg ttc tgc att gac ttc tct atg aag atg ctt gcc aag aat tct tat ttt cat tat tta tta aat atc ctg gtc atc ctg act ctt atc tta aat tgc tat caa act aat ctg att tta ttt cct tga cca cag aca aat att gtt cta tac ttg tet aaa gta aat tgc agt att acc tat aac tct ttt tag ata ttt tag cag tta tat ttt tcc ttt ttt atc cta ctt agt tgt gat tct tga get tta tca gta ata tat atg ata aat ata aag tat ttt acc ctt atg aaa taa agt ttt aca caa agc aga atg tta caa ttg gct tta gtg ttg tat tta tgt agc tag aaa ctt att ttt tta aca tcc tgg aaa tat aca ata ttg ggt tcc atg cca aaa tat ttc caa aca aaa ctg tac acc tat ttt gtg get gca ctg agt ttg tga aat ctc tca tat ctt tct gat cat aac tgc atc tat gaa aag tat gag aaa gtg att tga gtg ctg agg aaa gaa tat aa ata ttc act cat tgt taa gaa gga att caa aaa cat gag gtt agt tga aaa tgg gtc tca gag $\mathrm{ccg}$ agt ttc att acc caa cta ggt aac atc atc agt gca gtt tt ctc tga act aac aat att ctc ttc tet tgc ttc tcc atc tct gat cat cet tet cac att gtt tta cag A GGA TTT TTT ACG CTT CGT GGG GAG ACG TAC TTA ATT GAA CCC TTG AAG $\begin{array}{lllllllllllllllll}G & F & F & T & L & R & G & E & T & Y & L & I & E & P & L & K\end{array}$ GTT CCC GAC AGT GAA TCC CAT GCA GTC TAC AAA TAT GAA GAT GCC AAA AAA AAG GAT GAG Prodom 1 Rv/Prodom $1 \mathrm{FW}$ $\begin{array}{cccccccccccccccccccc}\text { V } & \text { P } & \text { D } & \text { S } & \text { E } & \text { S } & \text { H } & \text { A } & \text { V } & \text { Y } & \text { K } & \text { Y } & \text { E } & \text { D } & \text { A } & \text { K } & K & K & \text { D } & \text { E }\end{array}$ $\begin{array}{lllllllllllllllllllll}A & P & K & M & C & G & V & T & L & T & N & W & E & S & D & K & P & I & K & K\end{array}$ GCT TCT CAT TTA GTT GCT ACT TCT GAA gta agt ctc ata ata aac ata gtt taa gat tac $\begin{array}{llllllllllll}\text { A } & \text { S } & \text { H } & \text { L } & \text { V } & \text { A } & \text { T } & \text { S } & \text { E } & \text { EoPI-6 }\end{array}$

ata cta att tcc ttg tct tga aaa tat aaa gta aga gag aat ttc ctt tgg gaa ggg gtg ata gat aga att caa aag gga gaa gcc ccc att tct ata ttt tta ttg tag cca tgg cat aaa aga aag aat gga aac ttg agg aac aga aa tac att ttc cag gct tat agc att ttc ttt ggt cat tca aac tta gtt tag aga ttt gaa tca aaa tct att taa atg agt ttc taa att atc tct agt ttc taa gtc aat gtt gaa aag taa tta aat tat caa ttt gga ttc ctc ttt tat gca tgc aga gag gat ggg gga caa agt ggt ttg aaa tat taa atg gtt tta aga tgt ctg ata agg cca tta cat aat tgt tac tcc att atc caa ttt gat ttg aat cat caa gtt gga ttg atg caa tga atg gat gaa aag tga caa tgt gaa cct agt cac aat tga ccc tta tgc tct caa tat ttt cct cct tta ttg gac gca caa aaa tta gaa aac aaa ata ttg cat cca aag tga cag ttc ctt tcc atc ttt ttg gtg gca aaa gtt gaa act ggc tga aaa atc tct act gtt tt att aga atg tta aaa ttg aca TGG AAC AAC AGC TGT TGT TAT GAC IntronB7PI Fw

Gga ata cca aaa cac aag tga aga cgc caa atg aag cet ggt ttg tct ttt ggc ttc ttt cat tct ggc aat tca aga ttc ttt atc ctc agc aat ttg tgg tta tac gtt aca ttt aac

Figure A2. Cont. 
cca gct atg gat cag ttt tgg att tct tct gct aaa gcc tga aga ctt tgt tgc ctc cta ttt cat gca atg aat agg agc cca gta aat atg gag aat atc aca tag cca ttc ctg cag tgg cgt agc atg ggg gtg cag ggg ggg cag ccg cac cgg gca caa cat ctg ggg ggg cgc get cgc act $\mathrm{cgc}$ agc tct ctg ccc ctg cet ggc tca ctc att ctc tct cca ctg aga aac cac gec gga ttc ccc tca cac gac cac tca cCC ggg aaa gec gag cga get cgc ccc acc ttt tcg agc ctt ttc tct cat ctc cag cet gtt ggc aac cgc aaa ttg ttt tga gcc ctg ttc tct tct ctc ccc ccc cgg ceg tgt taa gec aag gac aaa ctt tgc aag aaa ttg cag ttt tgc ttt ctc ttc ttc ccc ctc ccc tct ccc gta gag tag tgg ggg aaa ggg aat gtg gga gat ttg cca gcg gac aca gac ttt cca cta aac tcc cec cgc ctg gca tct cca cct cat ttt GGC CGC GTG AAT GCA TCT GCT TCt ttc tet ctt ctc acc cCa CCC caC cat cCa

\section{IntrF2PI Fw}

ctc gtg aaa agg gag ggg gag gtg cta ata cct gga aag aaa cta act ttc att tgc caa ttt cat aaa tgg tgg agt taa aga gag ttg ctg gaa aat tat ata tag tta cac gtt cgg ttt gtg tga gga aaa caa agt gaa tgc taa ttc ctt caa ggg ggt aat ttc ttt cca gct gaa ctg act agc cca tct atg agc cag tgt tgt tta aag aaa act aaa ttt aca aag aaa tct gtt gag aaa ttc tat tga ttc tga gca tat ttc atg ggg gca aag agg aga aat tag atc tct tga ctc ttt cag aat ctt get cet ttg tac act tct tta taa cag cac tgg gcc tgt gaa agc agg cga gaa gtc cta gag aag cta cca ttt caa tgc aga att cgc gac cca tta aac cta ttc ttt tat ggc agc ttc acc aca aac agc agc ttc tcc att ctc tac agg cag aaa aaa aat ggg aga ggg gca ttc atg cat cat ttc ctg aaa gaa tct ata cta aag aaa gta tgt aaa gcg ttg tct atc aaa aac tta ttc aaa agt tac gta acc aag gga tta tga att tgc agc aaa ttt gcc tat gaa ttt gga gta aat ttc ttt gta ggg tag caa aat gtg aca cca cta tat aca tat ata cag ggt tgg cca aaa tct gaa aag gaa tat agt cta tga aga gtt ata gac aaa tta aGC ATC AGT TTG TTC GCA CTC AAT AAA Gtg ttt gaa aat Intr2F2PI Fw

aaa ctt gta ttt aga tgc att tta ctt taa tta cat cag tat ttt cac aac aaa caa tac atg tgc tta ggg ggt aag ggt ttt ttt aac taa tct agt gga aga gac ttg agt gct aaa 1 microsatellite

atc cac ggg tta ggg ggc gca aat tac ttg cet tgc ccc agg tgc tga caa ccc atg cta cgc cac tgc att cet gtt ctt cct gaa gaa tgt ctg ggc atc acg ctt act ctt taa tag ttc tag aca ctt tgt att gta ttg tac aga gga gtt tgc tta gaa aac aat ttt tct caa tta acc cca cca aaa ggg tct gct gca act ttg act ttg gaa gaa gac cgc att gtt tgt tta atg gcc acc agc aga ttc tct ttg cat tct tgt ttc tac ccc tta agt tga ggg cca atg atg ata ttc cta atg att tga att aag tta gaa tgc cat gtt tgc atc tgt tta agt att cag ctt cag agt tca gtg gca tgt cgc tga tat tat cac ttt tca acc aaa gaa atc aat tag aaa tcc ttt aag gta aat aga aat tta gca tat tta get ctc tct tca tcc tta taa aag gtc cta tcc ctt caa aag aag aaa aga ata act gga tca aaa tta cac agt ttt ccc ttc aaa tat att att gtc ctc cca cag aga ggc tga cta ata atg aaa ata aaa ggc agc cca ttc aaa cat aag acg cag ctc gat caa cta att tca cat gga ttt aaa tgt aaa tgg att tgc taa aaa aag aaa gga aag tta gag aaa tta aaa ctc agg GAG CAT AAT CTG GAA CTA AGA TCA AGg ggt ata aag tat tct ctt atc ttt tet gtt agc ttt aga aga aaa Intr3F2PI Fw

aaa aat cac tgc att tct tct tta gca att cct ttt cct tat atg ttt ttg aaa tga att ttg ttc cta gtc tga att tgg agg atg tca tga tct ttt ttc cac ttc tac ag G GCA

CAA GAT TCC TAT CAC TTC AGT GAT TGT AGT AAG AAT GAA TAT CAG AGC TAT ATT GCT ACT

$\begin{array}{llllllllllllllllllllll}2 & \mathrm{D} & \mathrm{S} & \mathrm{Y} & \mathrm{H} & \mathrm{F} & \mathrm{S} & \mathrm{D} & \mathrm{C} & \mathrm{S} & \mathrm{K} & \mathrm{N} & \mathrm{E} & \mathrm{Y} & \mathrm{Q} & \mathrm{S} & \mathrm{Y} & \mathrm{I} & \mathrm{A} & \mathrm{T}\end{array}$ TAT AAC CCA CAG TGC ATT CTC AAT CAA CCC TTG AGA ACA GAT ACT GTT TCA ACT CCA GTT $\begin{array}{llllllllllllllllllllll} & Y & N & P & Q & C & I & L & N & Q & P & L & R & T & D & T & V & S & T & P & V\end{array}$ TCT GAA AAT GAA CTT TTG CAG gta gga gaa gaa tgt gac tgt ggc ttt cct gca tta agt $\begin{array}{lllllllllllllllll}\text { Met13PI Rv } & V & G & E & E & C & D & C & G & F & P & A & \mathrm{~L} & \mathrm{~S}\end{array}$

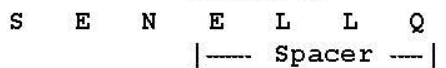

ctt ttt ttt taa tca aca aaa gta att tga aga ata ttc tca gaa atg aga atc ctt gaa L $\quad$ F $\quad$ F $S T O P$

aaa tca tct agc ttt cta agt ggt ttg agc cat cca aga ggt tgg ctt gtg aat ggc tga ggt ttg tgc ctt tca tgt aca tgc atg tat gaa atg gtt tct tgg gtt gta gag gaa tag aga aat ggt atc tca cta cta ttt ggg gaa gat ggt gaa ttt tta aaa agg ggt gat tga cca ttc cat gaa aat ctt tcc ctc ctg aaa acc cct att ttg ttg ata tag cca cat tat cct gtc cca caa tet tct cga act get cct tcc cat atc tga tta tct tta atc tat gct ctg atc cta ata ata ttt tta taa gaa cag taa tat agt gtt ttt atg ttg tta aat aca cct gta atg gtc tgt gag aat gtc ctt aag aga caa aag aag gag gaa aca tcc agt cag tgg tca tat aaa aag gag att aac ctg cag aaa caa agg ggc ata gca aaa atc tca aga ggg aca cct cct acc cat tct ctt ggt ccg taa aga tga ggt ggt aga aat gga ctt tca gta ttg aaa gat tct get act gta act gta caa tca agg tag tgt taa tgc tca tgg ttg gtg ctt ctt ctc tgg att acc tca aaa gct ggc atg atg agt aga aaa tct ctc atg aat aag aaa gga atg ggg ctg tag gtt atg tgg gct tca caa tta gga gat gag gat att ttt gtt tta ttc ttt tca cgt agg aaa tat cag ata agg ctc ttt cgc aga gaa atg cca tta tct gtt ttc aat aac aaa caa ttt ttg cat ttg cta gca tga acc cat aaa agg gaa cac att gca gaa att tcc ctc ttc aaa ata gac caa tta aaa aag aaa att cta tgc cat cat th ata tga tat gat ccg tgc tgt gat cct ata acg tgt aaa cca aga caa ggg aaa cat $\begin{array}{ccccccccccccccccccc}* & & Y & D & P & C & C & D & P & I & T & C & K & P & R & Q & G & K & H \\ \text { tgt } & & & \end{array}$ $\begin{array}{llllllllllllllllllllllllll}C & V & S & G & L & C & C & C & S & Y & K & V & R & L & V & Y & F & *\end{array}$

gag aaa ttt tac cet get cca tac tag cca tgt aga aat gta ata tet ctt ggc ttt tta

Figure A2. Cont. 
tgt ttt aaa ctt ct ata aaa tta tct ctg tgc tac ata at cet gat ata gat atc tct tet ttg cat tet ttc cag A GGA CTT TTT TCA GAA GAT TAC AGT GAG ACT CAT TAT TCC Pro2 Fw/Pro3 Rv

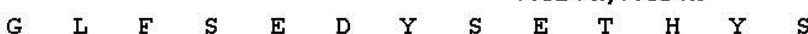

CCT GAT GGC AGC GAA ATT ACA ACA AAC CCT CCT GTT GAG gta ggg tct cac ttt tat gag

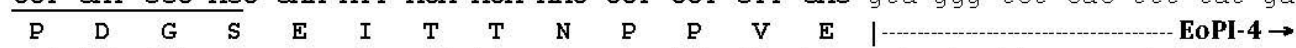
cet ttt tt aag gaa gta aat tga aac aaa tgt ttg tgc act ata tta caa ata tac aag aat gag acc agg cta ctc aaa caa agt gta tat aag tat aaa gta tct tat att gat atg tac tta caa aga tgc ctg gat tgt taa tcc ttg gtt aaa agc caa cat att tgg gag gtg agt ttc aca aat aga ttt att atg aga aca tca ggt ttg taa gat tat att ttc att ttt aaa cca gac tac agg gat aaa tgc aaa gtc ttt tat ctg taa tac caa aag tga taa caa ttc act ttg ctc cta tac aga aat cca ttt aac atc tt cat att aaa atg gtg cca aaa atg get cta tca gag gtt aaa aaa tta cag cac taa tat gct tca tgt tgg ctc cat ttc $\mathrm{CCC}$ aaa ttg att taa aag tgc att ctg tgt cta ttt ctg gtt tag cat ctt cat ggg ttg cac aaa tta ctc ctt tgt $g c c$ atc agt ggc act ctc cca tag tgt gac ttg att tat gga gac ttg cat tta tcc tat gtt cct tt gca ata gtc agt at aag aag gtt ttc tgt cct cct gaa tca aaa tt tct gga aaa ctg ctg tct aaa tat ttc att gat gtt atg gaa tac att gga act gta ctt ctg ctc atc aaa tca caa tac aaa gcc tta acc agt gta gtc ctc TTG CCT CCC TAT AG GAT CAC TGC TAT TAT TAT GGA CGC ATC CAG AAT GAT GCT GAC TCA

\section{IntrB13-1 Fw/Met16PI Rv}

$$
\begin{array}{lllllllllllllllll}
D & H & C & Y & Y & Y & G & R & I & Q & \text { N } & D & A & D & S
\end{array}
$$

ACT GCA AGC ATC AGC ACA TGC AAT GGT TTG AA gta aga tag tct cta atc ttt tat ttg $\begin{array}{lllllllllllll}T & A & S & I & S & T & C & N & G & L & K & & \end{array}$

ttt att aat aat aat ata gtg ctc ttg gag ttc taa ttg tta aaa tga agg aca tcc tca gtt ttt cat gga aat tag ttg ggt gtg atc cag gat ttc ggc aga att aag aca tac tt ggt tga aaa cca aga aga gct gct gcc agc cag gag aaa aac tat gga gct aaa tca cat aag tct aaa gga gct tcc aag ccc cgg tct cct ttc cca ggg tga ggt gat att aca ggt aga gaa gat tag tag gtt tca aat tgg aga cct tgc tag aaa gtg tac agg aag agg caa gaa gtt tca gtt cta ccc aga aac act ttc ttg agt cac tct gca cac tet ctt cag cca act aga tat gtt aac tac ata aag atc cca gaa ttc aga agg tcc cta tca ata gta aga atg aac atc acc tca aca tct ttt act gaa aaa aga cac tga aac tca cct ttg aac aga gac tgt gtc cat gga gtg gag gaa taa atg aaa agc tgg aac aga gca gaa taa caa cag aaa aat aaa gga aaa aca gaa tga cag aat aat agc att gga agg gac ttt gag gtc ttc

$$
\text { LINE/L2/CR1 } \rightarrow
$$

tag tcc aac ttc ctg ctc aag tag gag acc tat atc atc cta gac aaa tag ctg tca atc ttc tct taa aaa gca gta gtg atg gaa cac cca caa tgt ctg aat agg tta att gtt cca ttt gtg aga aaa tta ctc ctt agt tct aac tta ttt ctc tct ttg gtt act ttc cac gca ttg ctt ctt ctc ctg cca tca ggt gaa gaa tag gtt gtc cca cat ttt tta tga cag cct ctt aaa tac tta aag att atc aag tca tct cta ccc ctt ctt gtc act agc atg agt ata ctc att gtc tgc agc cat tct aac ctc cag tta gta tgc att ctt att cct tca ttg tta ctc ctg ttg ttc tgc att gac ttc tct atg aag atg ctt gcc aag aat tct tat ttt cat tat tta tta aat atc ctg gtc atc ctg act ctt atc tta aat tgc tat caa act aat ctg att tta ttt cet tga cca cag aca aat att gtt cta tac ttg ttt aaa gta aat tgc agt att acc tat aac tct ttt tag ata ttt tag cag tta tat ttt tcc ttt ttt atc cta ctt agt tgt gat tct tga get tta tca gta ata tat atg ata aat ata aag tat ttt acc ctt atg aaa taa agt ttt aca caa agc aga atg tta caa ttg gct tta gtg ttg tat tta tgt agc tag aaa ctt att ttt tta aca tcc tgg aaa tat aca ata ttg ggt tcc atg cca aaa tat ttc caa aca aaa ctg tac acc tat ttt gtg get gca ctg agt ttg tga aat ctc tca tat ctt tct gat cat aac tgc atc tat gaa aag tat gag aaa gtg att tga gtg ctg agg aaa gaa tat aa ata ttc act cat tgt taa gaa gga att caa aaa cat gag gtt agt tga aaa tgg gtc tca gag $\mathrm{ccg}$ agt ttc att acc caa cta ggt aac atc atc agt gca gtt tt ctc tga act aac aat att ctc ttc tet tgc ttc tcc atc tct gat cat cet tet cac att gtt tta cag A GGA TTT TTT ACG CTT CGT GGG GAG ACG TAC TTA ATT GAA CCC TTG AAG $\begin{array}{lllllllllllllllll}G & F & F & T & L & R & G & E & T & Y & L & I & E & P & L & K\end{array}$ GTT CCC GAC AGT GAA TCC CAT GCA GTC TAC AAA TAT GAA GAT GCC AAA AAA AAG GAT GAG Prodom 1 Rv/Prodom $1 \mathrm{FW}$ $\begin{array}{cccccccccccccccccccc}\text { V } & \text { P } & \text { D } & \text { S } & \text { E } & \text { S } & \text { H } & \text { A } & \text { V } & \text { Y } & \text { K } & \text { Y } & \text { E } & \text { D } & \text { A } & \text { K } & K & K & \text { D } & \text { E }\end{array}$ $\begin{array}{lllllllllllllllllllll}A & P & K & M & C & G & V & T & L & T & N & W & E & S & D & K & P & I & K & K\end{array}$ GCT TCT CAT TTA GTT GCT ACT TCT GAA gta agt ctc ata ata aac ata gtt taa gat tac $\begin{array}{llllllllllll}\text { A } & \text { S } & \text { H } & \text { L } & \text { V } & \text { A } & \text { T } & \text { S } & \text { E } & \text { EoPI-6 }\end{array}$

ata cta att tcc ttg tct tga aaa tat aaa gta aga gag aat ttc ctt tgg gaa ggg gtg ata gat aga att caa aag gga gaa gcc ccc att tct ata ttt tta ttg tag cca tgg cat aaa aga aag aat gga aac ttg agg aac aga aa tac att ttc cag gct tat agc att ttc ttt ggt cat tca aac tta gtt tag aga ttt gaa tca aaa tct att taa atg agt ttc taa att atc tct agt ttc taa gtc aat gtt gaa aag taa tta aat tat caa ttt gga ttc ctc ttt tat gca tgc aga gag gat ggg gga caa agt ggt ttg aaa tat taa atg gtt tta aga tgt ctg ata agg cca tta cat aat tgt tac tcc att atc caa tet gat ttg aat cat caa gtt gga ttg atg caa tga atg gat gaa aag tga caa tgt gaa cct agt cac aat tga ccc tta tgc tct caa tat ttt cct cct tta ttg gac gca caa aaa tta gaa aac aaa ata ttg cat cca aag tga cag ttc ctt tcc atc ttt ttg gtg gca aaa gtt gaa act ggc tga aaa atc tct act gtt tt att aga atg tta aaa ttg aca TGG AAC AAC AGC TGT TGT TAT GAC IntronB7PI Fw

Gga ata cca aaa cac aag tga aga cgc caa atg aag cet ggt ttg tct ttt ggc ttc ttt cat tct ggc aat tca aga ttc ttt atc ctc agc aat ttg tgg tta tac gtt aca ttt aac

Figure A2. Cont. 


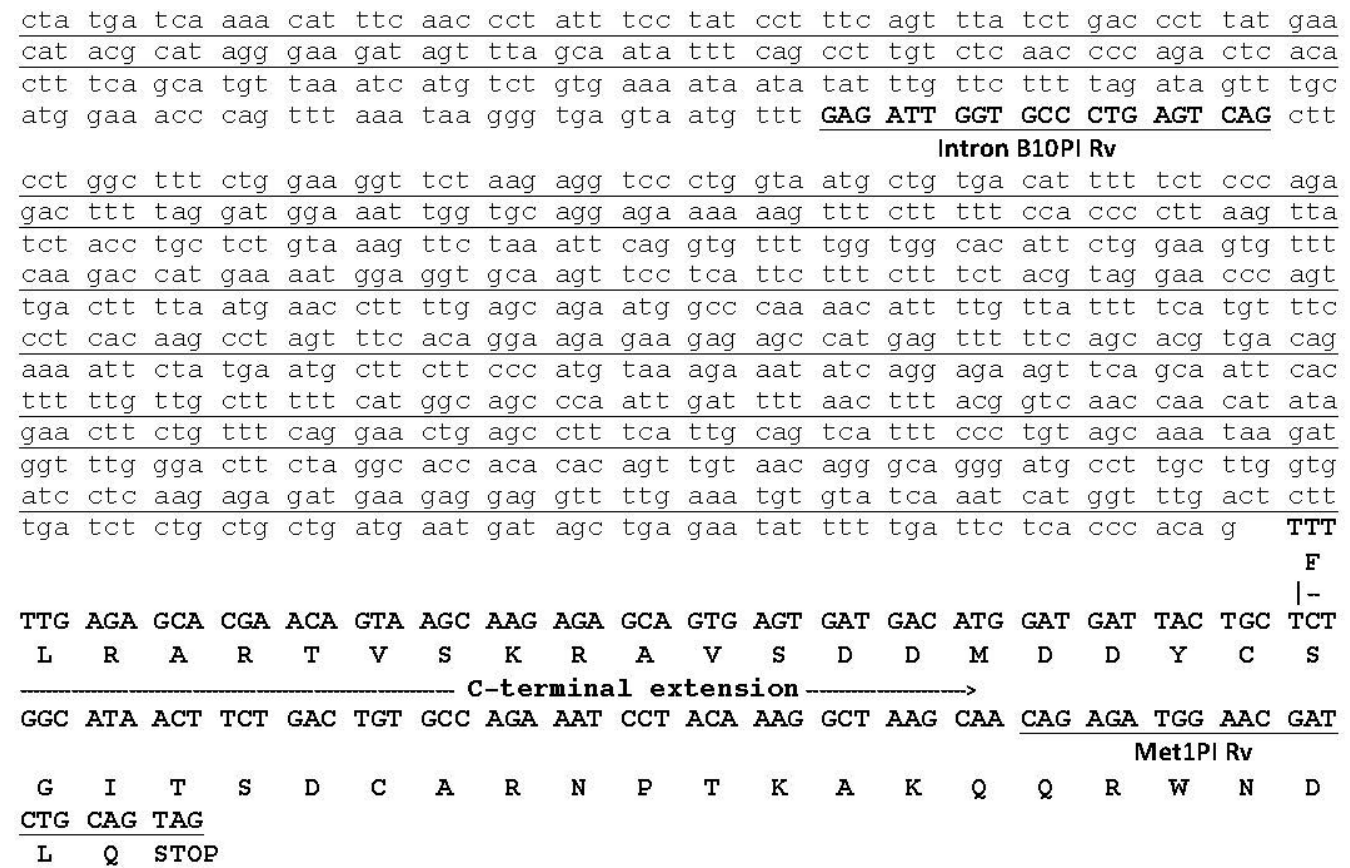

Figure A2. Genomic organization of E. ocellatus EOC00028-like PI-SVMP gene.

\section{References}

1. MEROPS- the Peptidase Database: Family M72. Available online: https://merops.sanger.ac.uk/cgi-bin/ famsum?family=M12 (accessed on 8 May 2016).

2. PFAM Family: REprolysin. Available online: http://pfam.xfam.org/family/PF01421 (accessed on 8 May 2016).

3. Seals, D.F.; Courtneidge, S.A. The ADAMs family of metalloproteases: Multidomain proteins with multiple functions. Genes Dev. 2003, 17, 7-30. [CrossRef] [PubMed]

4. Giebeler, N.; Zigrino, P. A disintegrin and metalloprotease (ADAM): Historical overview of their functions. Toxins 2016, 8. [CrossRef] [PubMed]

5. Arendt, D.; Technau, U.; Wittbrodt, J. Evolution of the bilaterian larval foregut. Nature 2001, 409, 81-85. [CrossRef] [PubMed]

6. Tucker, R.P.; Adams, J.C. Adhesion Networks of Cnidarians: A Postgenomic View. Int. Rev. Cell Mol. Biol. 2014, 308, 323-377. [PubMed]

7. Bahudhanapati, H.; Bhattacharya, S.; Wei, S. Evolution of Vertebrate Adam Genes; Duplication of Testicular Adams from Ancient Adam9/9-like Loci. PLoS ONE 2015, 10, e0136281. [CrossRef] [PubMed]

8. Cho, C. Testicular and epididymal ADAMs: Expression and function during fertilization. Nat. Rev. Urol. 2012, 9, 550-560. [CrossRef] [PubMed]

9. Bates, E.E.; Fridman, W.H.; Mueller, C.G. The ADAMDEC1 (decysin) gene structure: Evolution by duplication in a metalloprotease gene cluster on chromosome 8p12. Immunogenetics 2002, 54, 96-105. [CrossRef] [PubMed]

10. Wei, S.; Whittaker, C.A.; Xu, G.; Bridges, L.C.; Shah, A.; White, J.M.; Desimone, D.W. Conservation and divergence of ADAM family proteins in the Xenopus genome. BMC Evol. Biol. 2010, 10. [CrossRef] [PubMed]

11. Taylor, J.S.; Raes, J. Duplication and divergence: The evolution of new genes and old ideas. Annu. Rev. Genet. 2004, 38, 615-643. [CrossRef] [PubMed]

12. Ohno, S. Evolution by Gene Duplication; Springer-Verlag: Berlin-Heidelberg, Germany, 1970.

13. Zhang, J. Evolution by gene duplication: An update. Trends Ecol. Evol. 2003, 18, 292-298. [CrossRef]

14. True, J.R.; Carroll, S.B. Gene co-option in physiological and morphological evolution. Annu. Rev. Cell Dev. Biol. 2002, 18, 53-80. [CrossRef] [PubMed] 
15. Kaessmann, H.; Vinckenbosch, N.; Long, M. RNA-based gene duplication: Mechanistic and evolutionary insights. Nat. Rev. Genet. 2009, 10, 19-31. [CrossRef] [PubMed]

16. Vinckenbosch, N.; Dupanloup, I.; Kaessmann, H. Evolutionary fate of retroposed gene copies in the human genome. Proc. Natl. Acad. Sci. USA 2006, 103, 3220-3225. [CrossRef] [PubMed]

17. Fry, B.; Wüster, W. Assembling an Arsenal: Origin and evolution of the snake venom proteome inferred from phylogenetic analysis of toxin sequences. Mol. Biol. Evol. 2004, 21, 870-883. [CrossRef] [PubMed]

18. Fry, B.G.; Vidal, N.; Norman, J.A.; Vonk, F.J.; Scheib, H.; Ramjan, S.F.; Kuruppu, S.; Fung, K.; Hedges, S.B.; Richardson, M.K.; et al. Early evolution of the venom system in lizards and snakes. Nature 2006, 439, 584-588. [CrossRef] [PubMed]

19. Fry, B.G.; Casewell, N.R.; Wüster, W.; Vidal, N.; Young, B.; Jackson, N. The structural and functional diversification of the Toxicofera reptile venom system. Toxicon 2012, 60, 434-448. [CrossRef] [PubMed]

20. Casewell, N.R.; Wüster, W.; Vonk, F.J.; Harrison, R.A.; Fry, B.G. Complex cocktails: The evolutionary novelty of venoms. Trends Ecol. Evol. 2013, 28, 219-229. [CrossRef] [PubMed]

21. Haney, R.A.; Clarke, T.H.; Gadgil, R.; Fitzpatrick, R.; Hayashi, C.Y.; Ayoub, N.A.; Garb, J.E. Effects of gene duplication, positive selection, and shifts in gene expression on the evolution of the venom gland transcriptome in widow spiders. Genome Biol. Evol. 2016, 8, 228-242. [CrossRef] [PubMed]

22. Wong, E.S.; Belov, K. Venom evolution through gene duplications. Gene 2012, 496, 1-7. [CrossRef] [PubMed]

23. Vonk, F.J.; Casewell, N.R.; Henkel, C.V.; Heimberg, A.M.; Jansen, H.J.; McCleary, R.J.; Kerkkamp, H.M.; Vos, R.A.; Guerreiro, I.; Calvete, J.J.; et al. The king cobra genome reveals dynamic gene evolution and adaptation in the snake venom system. Proc. Natl. Acad. Sci. USA 2013, 110, 20651-20656. [CrossRef] [PubMed]

24. Hedges, S.B.; Vidal, N. Lizards, snakes, and amphisbaenians (Squamata). In The Timetree of Life; Hedges, S.B., Kumar, S., Eds.; Oxford University Press: Oxford, UK, 2009; pp. 383-389.

25. Jones, M.E.; Anderson, C.L.; Hipsley, C.A.; Müller, J.; Evans, S.E.; Schoch, R.R. Integration of molecules and new fossils supports a Triassic origin for Lepidosauria (lizards, snakes, and tuatara). BMC Evol. Biol. 2013, 13. [CrossRef] [PubMed]

26. Pyron, R.A.; Burbrink, F.T.; Wiens, J.J. A phylogeny and revised classification of Squamata, including 4161 species of lizards and snakes. BMC Evol. Biol. 2013, 13. [CrossRef] [PubMed]

27. Reeder, T.W.; Townsend, T.M.; Mulcahy, D.G.; Noonan, B.P.; Wood, P.L., Jr.; Sites, J.W., Jr.; Wiens, J.J. Integrated analyses resolve conflicts over squamate reptile phylogeny and reveal unexpected placements for fossil. PLoS ONE 2015, 10, e0118199. [CrossRef] [PubMed]

28. Hsiang, A.Y.; Field, D.J.; Webster, T.H.; Behlke, A.D.; Davis, M.B.; Racicot, R.A.; Gauthier, J.A. The origin of snakes: Revealing the ecology, behavior, and evolutionary history of early snakes using genomics, phenomics, and the fossil record. BMC Evol. Biol. 2015, 15. [CrossRef] [PubMed]

29. Reeks, T.A.; Fry, B.G.; Alewood, P.F. Privileged frameworks from snake venom. Cell. Mol. Life Sci. 2015, 72, 1939-1958. [CrossRef] [PubMed]

30. Hite, L.A.; Jia, L.G.; Bjarnason, J.B.; Fox, J.W. cDNA sequences for four snake venom metalloproteinases: Structure, classification, and their relationship to mammalian reproductive proteins. Arch. Biochem. Biophys. 1994, 308, 182-191. [CrossRef] [PubMed]

31. Moura-da-Silva, A.M.; Theakston, R.D.; Crampton, J.M. Evolution of disintegrin cysteine-rich and mammalian matrix-degrading metalloproteinases: Gene duplication and divergence of a common ancestor rather than convergent evolution. J. Mol. Evol. 1996, 43, 263-269. [CrossRef] [PubMed]

32. Casewell, N.R. On the ancestral recruitment of metalloproteinases into the venom of snakes. Toxicon 2012, 60, 449-454. [CrossRef] [PubMed]

33. Escalante, T.; Rucavado, A.; Fox, J.W.; Gutiérrez, J.M. Key events in microvascular damage induced by snake venom hemorrhagic metalloproteinases. J. Proteomics 2011, 74, 1781-1794. [CrossRef] [PubMed]

34. Markland, F.S., Jr.; Swenson, S. Snake venom metalloproteinases. Toxicon 2013, 62, 3-18. [CrossRef] [PubMed]

35. Herrera, C.; Escalante, T.; Voisin, M.B.; Rucavado, A.; Morazán, D.; Macêdo, J.K.; Calvete, J.J.; Sanz, L.; Nourshargh, S.; Gutiérrez, J.M.; et al. Tissue localization and extracellular matrix degradation by PI, PII and PIII snake venom metalloproteinases: Clues on the mechanisms of venom-induced hemorrhage. PLoS Negl. Trop. Dis. 2015, 9, e0003731. [CrossRef] [PubMed]

36. Gutiérrez, J.M.; Escalante, T.; Rucavado, A.; Herrera, C. Hemorrhage Caused by Snake Venom Metalloproteinases: A Journey of Discovery and Understanding. Toxins 2016, 8. [CrossRef] [PubMed] 
37. Jia, L.G.; Shimokawa, K.; Bjarnason, J.B.; Fox, J.W. Snake venom metalloproteinases: Structure, function and relationship to the ADAMs family of proteins. Toxicon 1996, 34, 1269-1276. [CrossRef]

38. Pyron, R.A.; Burnbrink, F.T. Extinction ecological opportunity and the origins of global snake diversity. Evolution 2012, 66, 163-178. [CrossRef] [PubMed]

39. Fox, J.W.; Serrano, S.M. Structural considerations of the snake venom metalloproteinases, key members of the M12 reprolysin family of metalloproteinases. Toxicon 2005, 45, 969-985. [CrossRef] [PubMed]

40. Fox, J.W.; Serrano, S.M. Insights into and speculations about snake venom metalloproteinase (SVMP) synthesis, folding and disulfide bond formation and their contribution to venom complexity. FEBS J. 2008, 275, 3016-3030. [CrossRef] [PubMed]

41. Casewell, N.R.; Sunagar, K.; Takacs, Z.; Calvete, J.J.; Jackson, T.N.W.; Fry, B.G. Snake venom metalloprotease enzymes. In Venomous Reptiles and Their Toxins: Evolution, Pathophysiology and Biodiscovery; ISBN: 978-0-19-930939-9. Fry, B.G., Ed.; Oxford University Press: Oxford, UK, 2015; Chapter 23; pp. 347-363.

42. Juárez, P.; Comas, I.; González-Candelas, F.; Calvete, J.J. Evolution of snake venom disintegrins by positive Darwinian selection. Mol. Biol. Evol. 2008, 25, 2391-2407. [CrossRef] [PubMed]

43. Carbajo, R.J.; Sanz, L.; Pérez, A.; Calvete, J.J. NMR structure of bitistatin-A missing piece in the evolutionary pathway of snake venom disintegrins. FEBS J. 2015, 282, 341-360. [CrossRef] [PubMed]

44. Calvete, J.J. Brief History and Molecular Determinants of Snake Venom Disintegrin Evolution. In Toxins and Hemostasis. From Bench to Bedside; Kini, R.M., Markland, F., McLane, M.A., Morita, T., Eds.; Springer Science+Business Media B.V.: Amsterdam, The Netherlands, 2010; pp. 285-300.

45. Casewell, N.R.; Wagstaff, S.C.; Harrison, R.A.; Renjifo, C.; Wüster, W. Domain loss facilitates accelerated evolution and neofunctionalization of duplicate snake venom metalloproteinase toxin genes. Mol. Biol. Evol. 2011, 28, 2637-2649. [CrossRef] [PubMed]

46. Sanz-Soler, R.; Sanz, L.; Calvete, J.J. Distribution of RPTLN genes across Reptilia. Hypothesized role for RPTLN in the evolution of SVMPs. Integr. Compar. Biol. 2016. [CrossRef] [PubMed]

47. Sanz, L.; Harrison, R.A.; Calvete, J.J. First draft of the genomic organization of a PIII-SVMP gene. Toxicon 2012, 60, 455-469. [CrossRef] [PubMed]

48. Endo, T.; Fedorov, A.; de Souza, S.J.; Gilbert, W. Do Introns Favor or Avoid Regions of Amino Acid Conservation? Mol. Biol. Evol. 2002, 19, 521-525. [CrossRef] [PubMed]

49. Zhou, Q.; Wang, W. On the origin and evolution of new genes-a genomic and experimental perspective. J. Genet. Genomics 2008, 35, 639-648. [CrossRef]

50. Kordis, D.; Gubensek, F. Adaptive evolution of animal toxin multigene families. Gene 2000, 261, 43-52. [CrossRef]

51. Cao, Z.; Yu, Y.; Wu, Y.; Hao, P.; Di, Z.; He, Y.; Chen, Z.; Yang, W.; Shen, Z.; He, X.; et al. The genome of Mesobuthus martensii reveals a unique adaptation model of arthropods. Nat. Commun. 2013, 4. [CrossRef] [PubMed]

52. Fry, B.G.; Wüster, W.; Kini, R.M.; Brusic, V.; Khan, A.; Venkataraman, D.; Rooney, A.P. Molecular evolution and phylogeny of elapid snake venom three-finger toxins. J. Mol. Evol. 2003, 57, 110-129. [CrossRef] [PubMed]

53. Reyes-Velasco, J.; Card, D.C.; Andrew, A.L.; Shaney, K.J.; Adams, R.H.; Schield, D.R.; Casewell, N.R.; Mackessy, S.P.; Castoe, T.A. Expression of venom gene homologs in diverse python tissues suggests a new model for the evolution of snake venom. Mol. Biol. Evol. 2015, 32, 173-183. [CrossRef] [PubMed]

54. Hargreaves, A.D.; Swain, M.T.; Logan, D.W.; Mulley, J.F. Testing the Toxicofera: Comparative transcriptomics casts doubt on the single, early evolution of the reptile venom system. Toxicon 2014, 92, 140-156. [CrossRef] [PubMed]

55. Sunagar, K.; Jackson, T.N.; Undheim, E.A.; Ali, S.A.; Antunes, A.; Fry, B.G. Three-fingered RAVERs: Rapid Accumulation of Variations in Exposed Residues of snake venom toxins. Toxins 2013, 5, 2172-2208. [CrossRef] [PubMed]

56. Chang, D.; Duda, T.F. Extensive and continuous duplication facilitates rapid evolution and diversification of gene families. Mol. Biol. Evol. 2012, 29, 2019-2029. [CrossRef] [PubMed]

57. Chow, L.T.; Gelinas, R.E.; Broker, T.R.; Roberts, R.J. An amazing sequence arrangement at the $5^{\prime}$ ends of adenovirus 2 messenger RNA. Cell 1977, 12, 1-8. [CrossRef]

58. Berget, S.M.; Moore, C.; Sharp, P.A. Spliced segments at the $5^{\prime}$ terminus of adenovirus 2 late mRNA. Proc. Natl. Acad. Sci. USA 1977, 74, 3171-3175. [CrossRef] [PubMed] 
59. Bicknell, A.A.; Cenik, C.; Chua, H.N.; Roth, F.P.; Moore, M.J. Introns in UTRs: Why we should stop ignoring them. BioEssays 2012, 34, 1025-1034. [CrossRef] [PubMed]

60. Cenik, C.; Chua, H.N.; Zhang, H.; Tarnawsky, S.P.; Akef, A.; Derti, A.; Tasan, M.; Moore, M.J.; Palazzo, A.F.; Roth, F.P. Genome analysis reveals interplay between $5^{\prime}$-UTR introns and nuclear mRNA export for secretory and mitochondrial genes. PLoS Genet. 2011, 7, e1001366. [CrossRef] [PubMed]

61. Comeron, J.M.; Kreitman, M. The correlation between intron length and recombination in Drosophila: Dynamic equilibrium between mutational and selective forces. Genetics 2000, 156, 1175-1190. [PubMed]

62. De Souza, S.J.; Long, M.; Gilbert, W. Introns and gene evolution. Genes Cells 1996, 1, 493-505. [CrossRef] [PubMed]

63. Patthy, L. Exon shuffling and other ways of module exchange. Matrix Biol. 1996, 15, 301-310. [CrossRef]

64. Hughes, A.L.; Hughes, M.K. Small genomes for better flyers. Nature 1995, 377, 391. [CrossRef] [PubMed]

65. Lynch, M. Intron evolution as a population-genetic process. Proc. Natl. Acad. Sci. USA 2002, 99, 6118-6123. [CrossRef] [PubMed]

66. Haddrill, P.R.; Charlesworth, B.; Halligan, D.L.; Andolfatto, P. Patterns of intron sequence evolution in Drosophila are dependent upon length and GC content. Genome Biol. 2005, 6, R67. [CrossRef] [PubMed]

67. Zhao, M.; He, L.; Gu, Y.; Wang, Y.; Chen, Q.; He, C. Genome-wide analyses of a plant-specific LIM-domain gene family implicate its evolutionary role in plant diversification. Genome Biol. Evol. 2014, 6, 1000-1012. [CrossRef] [PubMed]

68. Tordai, H.; Patthy, L. Insertion of spliceosomal introns in proto-splice sites: The case of secretory signal peptides. FEBS Lett. 2004, 575, 109-111. [CrossRef] [PubMed]

69. Tomita, M.; Shimizu, N.; Brutlag, D.L. Introns and reading frames: Correlation between splicing sites and their codon positions. Mol. Biol. Evol. 1996, 13, 1219-1223. [CrossRef] [PubMed]

70. Long, M.; de Souza, S.J.; Rosenberg, C.; Gilbert, W. Relationship between proto-splice sites and intron phases: Evidence from dicodon analysis. Proc. Natl. Acad. Sci. USA 1998, 95, 219-223. [CrossRef] [PubMed]

71. Von Heijne, G. Patterns of amino acids near signal-sequence cleavage sites. Eur. J. Biochem. 1983, 133, 17-21. [CrossRef] [PubMed]

72. Pinho, C.; Rocha, S.; Carvalho, B.M.; Lopes, S.; Mourão, S.; Vallinoto, M.; Brunes, T.O.; Haddad, C.F.B.; Gonçalves, H.; Sequeira, F.; et al. New primers for the amplification and sequencing of nuclear loci in a taxonomically wide set of reptiles and amphibians. Conserv. Genet. Resour. 2010, 2, 181-185. [CrossRef]

73. Ellegren, H. Microsatellites: Simple sequences with complex evolution. Nat. Rev. Genet. 2004, 5, 435-445. [CrossRef] [PubMed]

74. Adams, R.H.; Blackmon, H.; Reyes-Velasco, J.; Schield, D.R.; Card, D.C.; Andrew, A.L.; Waynewood, N.; Castoe, T.A. Microsatellite landscape evolutionary dynamics across 450 million years of vertebrate genome evolution. Genome 2016, 59, 295-310. [CrossRef] [PubMed]

75. Liang, K.C.; Tseng, J.T.; Tsai, S.J.; Sun, H.S. Characterization and distribution of repetitive elements in association with genes in the human genome. Comput. Biol. Chem. 2015, 57, 29-38. [CrossRef] [PubMed]

76. Schlötterer, C.; Tautz, D. Slippage synthesis of simple sequence DNA. Nucleic Acids Res. 1992, 20, 211-215. [CrossRef] [PubMed]

77. Charlesworth, B.; Sniegowski, P.; Stephan, W. The evolutionary dynamics of repetitive DNA in eukaryotes. Nature 1994, 371, 215-220. [CrossRef] [PubMed]

78. Martin, P.; Makepeace, K.; Hill, S.A.; Hood, D.W.; Moxon, E.R. Microsatellite instability regulates transcription factor binding and gene expression. Proc. Natl. Acad. Sci. USA 2005, 102, 3800-3804. [CrossRef] [PubMed]

79. Li, Y.C.; Korol, A.B.; Fahima, T.; Beiles, A.; Nevo, E. Microsatellites: Genomic distribution, putative functions and mutational mechanisms: A review. Mol. Ecol. 2002, 11, 2453-2465. [CrossRef] [PubMed]

80. Shaney, K.J.; Schield, D.R.; Card, D.C.; Ruggiero, R.P.; Pollock, D.D.; Mackessy, S.P.; Castoe, T.A. Squamate reptile genomics and evolution. In Handbook. of Toxinology: Venom Genomics and Proteomics; Gopalakrishnakone, P., Calvete, J.J., Eds.; Springer Science+Business Media: Dordrecht, The Netherlands, 2016; pp. 29-49.

81. Balaresque, P.; King, T.E.; Parkin, E.J.; Heyer, E.; Carvalho-Silva, D.; Kraaijenbrink, T.; de Knijff, P.; Tyler-Smith, C.; Jobling, M.A. Gene conversion violates the stepwise mutation model for microsatellites in Y-chromosomal palindromic repeats. Hum. Mutat. 2014, 35, 609-617. [CrossRef] [PubMed] 
82. Eller, C.D.; Regelson, M.; Merriman, B.; Nelson, S.; Horvath, S.; Marahrens, Y. Repetitive sequence environment distinguishes housekeeping genes. Gene 2007, 390, 153-165. [CrossRef] [PubMed]

83. Sverdlov, E.D. Perpetually mobile footprints of ancient infections in human genome. FEBS Lett. 1998, 428, 1-6. [CrossRef]

84. Makalowski, W. Genomic scrap yard: How genomes utilize all that junk. Gene 2000, 259, 61-67. [CrossRef]

85. Bourque, G.; Leong, B.; Vega, V.B.; Chen, X.; Lee, Y.L.; Srinivasan, K.G.; Chew, J.L.; Ruan, Y.; Wei, C.L.; $\mathrm{Ng}, \mathrm{H} . \mathrm{H}$.; et al. Evolution of the mammalian transcription factor binding repertoire via transposable elements. Genome. Res. 2008, 18, 1752-1762. [CrossRef] [PubMed]

86. Irimía, M.; Rukov, J.L.; Penny, D.; Vinther, J.; García-Fernández, J.; Roy, S.W. Origin of introns by 'intronization' of exonic sequences. Trends Genet. 2008, 24, 378-381. [CrossRef] [PubMed]

87. Bazaa, A.; Juarez, P.; Marrakchi, N.; Bel Lasfer, Z.; El Ayeb, M.; Harrison, R.A.; Calvete, J.J.; Sanz, L. Loss of introns along the evolutionary diversification pathway of snake venom disintegrins evidenced by sequence analysis of genomic DNA from Macrovipera lebetina transmediterranea and Echis ocellatus. J. Mol. Evol. 2007, 64, 261-271. [CrossRef] [PubMed]

88. Bonen, L.; Vogel, J. The ins and outs of group II introns. Trends Genet. 2001, 17, 322-331. [CrossRef]

89. Dibb, N.J.; Newman, A.J. Evidence that introns arose at proto-splice sites. EMBO J. 1989, 8, $2015-2021$. [PubMed]

90. Logsdon, J.M., Jr. The recent origins of spliceosomal introns revisited. Curr. Opin. Genet. Dev. 1998, 8, 637-648. [CrossRef]

91. Lynch, M.; Richardson, A.O. The evolution of spliceosomal introns. Curr. Opin. Genet. Dev. 2002, 12, 701-710. [CrossRef]

92. Patel, A.A.; Steitz, J.A. Splicing double: Insights from the second spliceosome. Nat. Rev. Mol. Cell Biol. 2003, 4, 960-970. [CrossRef] [PubMed]

93. Rodríguez-Trelles, F.; Tarrío, R.; Ayala, F.J. Origins and evolution of spliceosomal introns. Annu. Rev. Genet. 2006, 40, 47-76. [CrossRef] [PubMed]

94. Luo, Y.; Li, C.; Gong, X.; Wang, Y.; Zhang, K.; Cui, Y.; Sun, Y.E.; Li, S. Splicing-related features of introns serve to propel evolution. PLoS ONE 2013, 8, e58547. [CrossRef] [PubMed]

95. Nakashima, K.; Ogawa, T.; Oda, N.; Hattori, M.; Sakaki, Y.; Kihara, H.; Ohno, M. Accelerated evolution of Trimeresurus. flavoviridis venom gland phospholipase $\mathrm{A}_{2}$ isozymes. Proc. Natl. Acad. Sci. USA 1993, 90, 5964-5968. [CrossRef] [PubMed]

96. Nakashima, K.; Nobuhisa, I.; Deshimaru, M.; Nakai, M.; Ogawa, T.; Shimohigashi, Y.; Fukumaki, Y.; Hattori, M.; Sakaki, Y.; Hattori, S.; et al. Accelerated evolution in the protein-coding regions is universal in crotalinae snake venom gland phospholipase $\mathrm{A}_{2}$ isozyme genes. Proc. Natl. Acad. Sci. USA 1995, 92, 5605-5609. [CrossRef] [PubMed]

97. Ikeda, N.; Chijiwa, T.; Matsubara, K.; Oda-Ueda, N.; Hattori, S.; Matsuda, Y.; Ohno, M. Unique structural characteristics and evolution of a cluster of venom phospholipase $\mathrm{A}_{2}$ isozyme genes of Protobothrops flavoviridis snake. Gene 2010, 461, 15-25. [CrossRef] [PubMed]

98. Chijiwa, T.; Ikeda, N.; Masuda, H.; Hara, H.; Oda-Ueda, N.; Hattori, S.; Ohno, M. Structural characteristics and evolution of a novel venom phospholipase $\mathrm{A}_{2}$ gene from Protobothrops flavoviridis. Biosci. Biotechnol. Biochem. 2012, 76, 551-558. [CrossRef] [PubMed]

99. Chijiwa, T.; Nakasone, H.; Irie, S.; Ikeda, N.; Tomoda, K.; Oda-Ueda, N.; Hattori, S.; Ohno, M. Structural characteristics and evolution of the Protobothrops elegans pancreatic phospholipase $\mathrm{A}_{2}$ gene in contrast with those of Protobothrops genus venom phospholipase A2 genes. Biosci. Biotechnol. Biochem. 2013, 77, 97-102. [CrossRef] [PubMed]

100. Koszul, R.; Fischer, G. A prominent role for segmental duplications in modeling eukaryotic genomes. C. $R$. Biol. 2009, 332, 254-266. [CrossRef] [PubMed]

101. Ohta, T. Simple model for treating evolution of multigene families. Nature 1976, 263, 74-76. [CrossRef] [PubMed]

102. Smith, G.P. Evolution of repeated DNA sequences by unequal crossover. Science 1976, 191, $528-535$. [CrossRef] [PubMed]

103. Juárez, P.; Wagstaff, S.C.; Sanz, L.; Harrison, R.A.; Calvete, J.J. Molecular cloning of Echis ocellatus disintegrins reveals non-venom-secreted proteins and a pathway for the evolution of ocellatusin. J. Mol. Evol. 2006, 63, 183-193. [CrossRef] [PubMed] 
104. GeneWise. Available online: http://www.ebi.ac.uk/Tools/Wise2/index.html (accessed on 31 May 2016).

105. Basic Local Alignment Search Tool (BLAST). Available online: http://blast.ncbi.nlm.nih.gov/Blast.cgi (accessed on 5 June 2016).

106. ClustalW2-Multiple Sequence Alignment. Available online: http://www.ebi.ac.uk/Tools/msa/clustalw2 (accessed on 5 June 2016).

107. RepeatMasker. Available on: http:/ / www.repeatmasker.org (accessed on 30 December 2015).

108. Genetic Information Research Institute. Available on: http:/ /www.girinst.org (accessed on 7 January 2016).

109. National Center for Biotechnology Information (NCBI) Database. Available on: http://www.ncbi.nlm.nih. gov (accessed on 10 January 2016).

(C) 2016 by the authors; licensee MDPI, Basel, Switzerland. This article is an open access article distributed under the terms and conditions of the Creative Commons Attribution (CC-BY) license (http://creativecommons.org/licenses/by/4.0/). 